COMMUNICATIONS IN

ANALYSIS AND GEOMETRY

Volume 11, Number 2, 263-341, 2003

\title{
Notions of Convexity in Carnot Groups
}

\author{
Donatella Danielli, Nicola Garofalo, and Duy-Minh Nhieu
}

\section{CONTENTS}

1. Introduction

2. Preliminaries

3. Strongly convex functions: A notion to abandon

4. Mildly convex functions: The appearance of horizontal planes

5. Weakly convex functions: The horizontal Hessian

6 . Weak convexity of the gauge in groups of Heisenberg type

7. Convexity of sets

8. Further properties of second derivatives of weakly convex functions

9. Basic properties of first derivatives of weakly convex functions: $L^{\infty}-L^{1}$ estimates

10. The horizontal Monge-Ampère equation

11. Yet another notion of convexity

References

\section{Introduction.}

In 1993 motivated by some interesting questions in the theory of fully nonlinear equations, we proposed the study of the following problem. Consider a stratified, nilpotent Lie group $\boldsymbol{G}$ of step $r$, with Lie algebra $\mathfrak{g}=V_{1} \oplus \ldots \oplus V_{r}$, and let $X_{1}, \ldots, X_{m}$ be a fixed orthonormal basis of the first layer $V_{1}$. Continue to denote with $X_{1}, \ldots, X_{m}$ the corresponding system of left-invariant vector fields on $\boldsymbol{G}$. Given a $m \times m$ matrix-valued function $g \rightarrow A(g)=\left(a_{i j}(g)\right)$ on $\boldsymbol{G}$ having real measurable entries $a_{i j}=a_{j i}$, suppose in addition that there exist $\nu>0$ such that

$$
\nu|\zeta|^{2} \leq \sum_{i, j=1}^{m} a_{i j}(g) \zeta_{i} \zeta_{j} \leq \nu^{-1}|\zeta|^{2},
$$

for a. e. $g \in \boldsymbol{G}$, and for every $\zeta \in \mathbb{R}^{m}$. The stratification of the Lie algebra carries a natural family of non-isotropic dilations $\Delta_{\lambda} \xi=\lambda \xi_{1}+\lambda^{2} \xi_{2}+\ldots+\lambda^{r} \xi_{r}$,

\footnotetext{
${ }^{1}$ Supported in part by NSF Grant No. DMS-0070492
} 
for any $\xi=\xi_{1}+\xi_{2}+\ldots+\xi_{r} \in \mathfrak{g}$. Let $Q=\sum_{j=1}^{r} j \operatorname{dim} V_{j}$ be the homogeneous dimension of $\boldsymbol{G}$ associated with such dilations.

Conjecture: Given a connected, bounded open set $\Omega \subset \boldsymbol{G}$, let $F \in L^{Q}(\Omega)$. Suppose that $u \in \mathcal{L}_{\text {loc }}^{2, Q}(\Omega) \cap C(\bar{\Omega})$ satisfy

$$
L u \stackrel{\text { def }}{=} \frac{1}{2} \sum_{i, j=1}^{m} a_{i j}\left\{X_{i} X_{j} u+X_{j} X_{i} u\right\} \geq F
$$

in $\Omega$. There exists a constant $C=C(\boldsymbol{G}, \nu, \Omega)>0$ such that

$$
\sup _{\Omega} u \leq \sup _{\partial \Omega} u^{+}+C\|F\|_{L^{Q}(\Omega)} .
$$

Here, $\mathcal{L}_{\text {loc }}^{2, Q}(\Omega)$ indicates the Sobolev space of functions $u \in L_{l o c}^{Q}(\Omega)$ having weak derivatives $X_{i} X_{j} u \in L_{l o c}^{Q}(\Omega)$. We note explicitly that

$$
L u=\operatorname{tr}\left\{A\left[X^{2} u\right]^{\star}\right\}
$$

where we have denoted by $\left[X^{2} u\right]^{\star}$ the symmetrized horizontal Hessian of $u$, see (1.3), or Section 5. Concerning the optimality of the $L^{Q}$ norm in the estimate (1.1), we refer the reader to [DGN4]. In the abelian case, when $\boldsymbol{G}=\mathbb{R}^{n}$ with the standard homogeneous dilations, one has $\mathfrak{g}=V_{1}=\mathbb{R}^{n}$, so that $Q=n$, and $\left[X^{2} u\right]^{\star}=D^{2} u$, the standard Hessian matrix of $u$. The above conjecture, in this situation, is in fact the celebrated geometric maximum principle of Alexandrov-Bakelman-Pucci (except that the matrix $\left(a_{i j}\right)$ need not be uniformly elliptic), see [A], [Ba1], [Pu], and also [Ba3].

Given the pervasive role that convexity plays in such principle, in order to advance our comprehension of the above conjecture it seems natural to examine appropriate notions of convexity in the non-abelian setting. This is the goal of the present paper.

We next give a description of our results. The notion of weakly $H$-convex function set forth in Definition 5.5 is central to our work. Sections 3 and 4 are devoted to justifying how we arrived to such notion starting from that of Pansu differential. A function on a Carnot group $u: G \rightarrow \mathbb{R}$ is called weakly $H$-convex if for any $g \in \boldsymbol{G}$ and every $\lambda \in[0,1]$ one has

$$
u\left(g \delta_{\lambda}\left(g^{-1} g^{\prime}\right)\right) \leq(1-\lambda) u(g)+\lambda u\left(g^{\prime}\right) \text { for every } g^{\prime} \in H_{g} .
$$

In (1.2) we have denoted by $\left\{\delta_{\lambda}\right\}_{\lambda>0}$ the non-isotropic group dilations defined by $\delta_{\lambda}=\exp \circ \Delta_{\lambda} \circ \exp ^{-1}$, see $(2.9)$, whereas the point $g \delta_{\lambda}\left(g^{-1} g^{\prime}\right)$ 
represents the twisted convex combination of $g$ and $g^{\prime}$, with base point $g$. The notation $H_{g}$ indicates the horizontal plane passing through $g$, see Definition 4.1. The collection of all such planes determines the horizontal subbundle generated by a given orthonormal basis of the Lie algebra $\mathfrak{g}$. The trajectories of the dilations $g \delta_{\lambda}\left(g^{-1} g^{\prime}\right)$ are in general quite twisted and they badly fail to be sub-unitary. They possess however the important redeeming feature that when the end-point $g^{\prime}$ belongs to the horizontal plane through $g$, then such trajectories are just straight line segments, at least at the level of the Lie algebra, see Proposition 4.4.

This leads us to our first main result, Theorem 5.12, which states that a function $u$ in the Folland-Stein class $\Gamma^{2}(\boldsymbol{G})$ is weakly $H$-convex if and only if its symmetrized horizontal Hessian

$$
\left[X^{2} u(g)\right]^{\star}=\frac{1}{2}\left\{X^{2} u(g)+X^{2} u(g)^{T}\right\},
$$

is positive semi-definite at every $g \in \boldsymbol{G}$. Here,

$$
X^{2} u(g)=\left(X_{i} X_{j} u(g)\right)_{i, j=1, \ldots, m} .
$$

This result constitutes the sub-Riemannian counterpart to the classical characterization of convexity for $C^{2}$ functions. In this perspective it represents a crucial witness of the appropriateness of the notion of weak convexity, and it is central to the rest of the paper.

In Section 6 we study an important example of non-smooth weakly $H$ convex function. In Theorem 6.8 we prove that the intrinsic gauge in any group of Heisenberg type is weakly $H$-convex. This is a deep property of such groups, intimately connected with their symmetries, see Theorem 6.6. It would be interesting to know whether such property extends to arbitrary Carnot groups of step 2, but we presently ignore the answer.

Section 7 is devoted to studying the convexity of sets. Definition 7.1 derives in a natural way from the notion of weakly $H$-convex function. With such definition, we prove that a function is weakly $H$-convex if and only if such is its epigraph as a subset of the product Carnot group $\mathcal{G}=\boldsymbol{G} \times \mathbb{R}$.

The main result of Section 8 is Theorem 8.1, which states that if an $L_{l o c}^{1}(\boldsymbol{G})$ function $u$ is weakly $H$-convex, then the entries of its symmetrized horizontal Hessian are signed Radon measure. This property extends the well-known classical analogue for convex functions in Euclidean space. It plays an important role in the development of a notion of Alexandrov generalized solution of the Monge-Ampère equation in the sub-Riemannian setting, see [DGN3]. 
The notion of weakly $H$-convex function evolved in a natural way, so to speak, from our desire to identify a class as wide as possible, while still retaining the basic properties connected with the notion of Pansu differentiability. Its appropriateness is proved by Theorem 5.12. Yet, such notion is a major source of complications when one tries to extend the basic theorems of convex analysis and new ideas are necessary. To clarify this point, we emphasize the main obstructions connected with Definition 5.5. (i) It involves information about the function $u$ only the lower-dimensional manifold $H_{g}$. (ii) Furthermore, such information is one-sided since we cannot exchange the role of the base point $g$ with that of the point $g^{\prime} \in H_{g}$. (iii) More importantly, failure of $H_{e}$ at being a subgroup of $\boldsymbol{G}$, results in failure of the crucial transitivity property : $g_{2} \in H_{g_{1}}, g_{1} \in H_{g_{o}}$, implies $g_{2} \in H_{g_{o}}$. This phenomenon of inferring conclusions on a set of full measure from information on lower-dimensional sets is fully present in the above conjecture and represents a major obstacle to overcome.

In Section 9 we introduce a new idea in this direction which we believe will prove important also in the study of the geometric AlexandrovBakelman-Pucci maximum principle conjectured above. First, we establish Theorem 9.1 which shows that a weakly $H$-convex continuous function is in fact Lipschitz continuous with respect to the sub-Riemannian metric of $\boldsymbol{G}$. Furthermore, there exists $C=C(\boldsymbol{G})>0$ such that the following estimate holds

$$
\|X u\|_{L^{\infty}\left(B\left(g_{o}, R\right)\right)} \leq \frac{C}{R}\|u\|_{L^{\infty}\left(B\left(g_{o}, 3 R\right)\right)},
$$

for any $g_{o} \in \boldsymbol{G}$ and every $R>0$. This result follows from a quantitative characterization of Lipschitz continuity which was obtained in [GN2], and by suitably slicing the gauge pseudo-ball $B\left(g_{o}, R\right)$ with horizontal planes.

The main result of the section, which we also consider the central result of the paper, is Theorem 9.2. The latter states that there exists an absolute constant $C=C(\boldsymbol{G})>0$ such that for any function $u$ weakly $H$-convex in $\boldsymbol{G}$ and continuous, one has

$$
\sup _{B(g, r)}|u| \leq C \frac{1}{|B(g, 5 r)|} \int_{B(g, 5 r)}|u| d g^{\prime}
$$

and

$$
\underset{B(g, r)}{e s s \sup _{B(g, 15 r)}}|X u| \leq \frac{C}{r} \frac{1}{|B(g, 15 r)|} \int_{B\left(g^{\prime} .\right.}
$$

The main trust of the inequalities (1.5), (1.6) is that their right-hand sides involve a set of full measure. The way we accomplish this result is to 
exploit in an ad hoc way the weak convexity of $u$ along the one-parameter family of horizontal planes $\left\{H_{\gamma(s)} \mid-1 \leq s \leq 1\right\}$, based at points of the straight-line "segment" $\gamma(s)=\exp s X_{1}, s \in[-1,1]$. Here, $X_{1}$ is the first element of an orthonormal basis of the first-layer $V_{1}$ of $\mathfrak{g}$ (the specific choice of $X_{1}$, rather than any other element of the basis is, of course, absolutely immaterial). The proof consists in first establishing a suitable control of $\phi(s)=u(\gamma(s))$ on the lower dimensional sets $B(\gamma(s), 1) \cap H_{\gamma(s)}$, and then average the resulting inequality in $s \in[-1,1]$. The crucial geometric fact which plays in our favor in this last step is that the union of all the regions $B(\gamma(s), 1) \cap H_{\gamma(s)}$ covers a butterfly which is of full measure.

One of the fundamental aspects of the theory of convex functions is their connection with geometry through the Monge-Ampère equation det $D^{2} u=$ $f$. Such fully nonlinear equation becomes elliptic on strictly convex functions. In this perspective, it is natural to ask if there is a corresponding fully nonlinear operator attached to the notion of weak convexity in the sub-Riemannian setting. We discuss this question in Section 10, where we introduce the notion of horizontal Gaussian curvature for both the graph of a function $u: G \rightarrow \mathbb{R}$, and for a smooth manifold $\mathcal{S}=\partial \Omega$ given as the level set $\Omega=\{g \in \boldsymbol{G} \mid \phi(g)<0\}$ of a defining function $\phi$. In the former case, we define the horizontal Gaussian curvature of the graph of $u$ at the point $\left(g_{o}, u\left(g_{o}\right)\right)$ as the classical Gaussian curvature of the graph of the restriction $U_{g_{o}}$ of $u$ to the horizontal plane $H_{g_{o}}$. Such notion also works well for a level set, provided that the point $g_{o} \in \mathcal{S}$ be non-characteristic. Such new point of view was suggested by our approach to the minimal surface equation in [DGN2], and to the horizontal Bernstein problem, formulated in the same paper. For level sets one encounters difficulties when the point $g_{o}$ is characteristic, and this requires a deeper analysis of the asymptotic properties of the horizontal principal curvatures. For these aspects we refer the reader to the papers [DGN2], [CGP].

A systematic study of the horizontal Monge-Ampère equation in Carnot groups

$$
\operatorname{det}\left[\left(X^{2} u\right)^{\star}\right]=F \text {, }
$$

will be carried in a forthcoming paper [DGN3]. We also introduce the notion of horizontal normal map and establish some of its properties, and we propose a notion of viscosity solution for the equation (1.7). The latter is inspired to the classical one of Crandall-Ishii-Lions [CIL], but, once again, it strongly reflects those new difficulties which are intrinsic to the notion of weak convexity. Section 10 ends with Theorem 10.9 which, in essence, states 
that non-isotropic gauge in a group of Heisenberg type is a fundamental generalized solution of the horizontal Monge-Ampère equation. This result is one further witness of the deep geometric role played by the gauge in these ambients.

Section 11 closes the paper. The most elementary properties of classical convex functions are their being locally bounded and continuous on their effective domain. Corresponding properties for weakly $H$-convex functions are far from obvious and are presently being investigated. The purpose of this section is to prove that by strengthening Definition 5.5, but of course without falling back into the notion of strong $H$-convexity, one obtains an "intermediate" class of convex functions which, remarkably, share with the classical ones the above mentioned properties. This implies in particular that the results in Sections 8 and 9 are valid generically for such functions, without any additional assumption.

Acknowledgments: It is a pleasure to acknowledge several helpful and stimulating conversations with Luca Capogna on the subject of this paper.

After this paper was submitted we had a conversation with Luis Caffarelli in which he brought to our attention that for the Heisenberg group $\mathbb{H}^{1}$ a notion of convexity essentially equivalent to our Definition 5.5 had been formulated by him in his 1997 NSF Proposal. We thank him for bringing this fact to our attention, and also for kindly sharing with us the notes from his proposal.

This paper was submitted in April 2002, and it was simultaneously posted to the server CVGMT of the Scuola Normale Superiore, Pisa. A related paper has been posted to the same server by Lu, Manfredi and Stroffolini in June 2002. The notion of convexity adopted in [LMS] is a reformulation of Caffarelli's, and thereby equivalent to our notion of weak $H$-convexity. Several results in [LMS] overlap with ours, but the methods of proof are different.

\section{Preliminaries.}

We begin by introducing the relevant ambients and their many properties. A Carnot group $\boldsymbol{G}$ of step $r$ is a simply connected Lie group whose Lie algebra $\mathfrak{g}$ admits a nilpotent stratification of step $r$. This means that $\mathfrak{g}=$ $V_{1} \oplus V_{2} \oplus \cdots \oplus V_{r}$, and that moreover $\left[V_{1}, V_{j}\right]=V_{j+1}$ for $j=1, \ldots, r-1$, whereas $\left[V_{1}, V_{r}\right]=\{0\}$. We assume that a scalar product $\langle\cdot, \cdot\rangle$ is given on $\mathfrak{g}$ for which the $V_{j}^{\prime} s$ are mutually orthogonal. We let $m_{j}=\operatorname{dim} V_{j}$, 
$j=1, \ldots, r$, and denote by

$$
N=m_{1}+\ldots+m_{r}
$$

the topological dimension of $\boldsymbol{G}$. The notation $\left\{X_{j, 1}, \ldots, X_{j, m_{j}}\right\}, j=1, \ldots, r$, will indicate a fixed orthonormal basis of the $j-t h$ layer $V_{j}$. Elements of $V_{j}$ are assigned the formal degree $j$. As a rule, we will use letters $g, g^{\prime}, g_{o}$ for points in $G$, whereas we will reserve the letters $Z, Z^{\prime}, Z_{o}$, for elements of the Lie algebra $\mathfrak{g}$. We will denote by

$$
L_{g_{o}}(g)=g_{o} g, \quad R_{g_{o}}(g)=g g_{o},
$$

respectively, the left- and right-translations on $\boldsymbol{G}$ by an element $g_{o} \in \boldsymbol{G}$. Recall that the exponential map exp $: \mathfrak{g} \rightarrow \boldsymbol{G}$ is a global analytic diffeomorphism [V]. It allows to define analytic maps $\xi_{i}: \boldsymbol{G} \rightarrow V_{i}, i=1, \ldots, r$, by letting $g=\exp \left(\xi_{1}(g)+\ldots+\xi_{r}(g)\right)$. The mapping $\xi: \boldsymbol{G} \rightarrow \mathfrak{g}$ defined by

$$
\xi(g)=\xi_{1}(g)+\ldots+\xi_{r}(g)
$$

is the inverse of the exponential mapping. For $g \in G$, the projection of the exponential coordinates of $g$ onto the layer $V_{j}, j=1, \ldots, r$, are defined as follows

$$
x_{j, s}(g)=<\xi_{j}(g), X_{j, s}>, \quad s=1, \ldots, m_{j} .
$$

Since Carnot groups of step 2 will play a special role it will be convenient to have a separate notation for the first two layers $V_{1}$ and $V_{2}$. For simplicity, we set $m=m_{1}, k=m_{2}$, and indicate

$$
\begin{aligned}
& X=\left\{X_{1}, \ldots, X_{m}\right\}=\left\{X_{1,1}, \ldots, X_{1, m}\right\}, \\
& Y=\left\{Y_{1}, \ldots, Y_{k}\right\}=\left\{X_{2,1}, \ldots, X_{2, k}\right\} .
\end{aligned}
$$

We continue to denote by $X$ and $Y$ the corresponding systems of leftinvariant vector fields on $\boldsymbol{G}$ defined by

$$
X_{j}(g)=\left(L_{g}\right)_{*}\left(X_{j}\right), j=1, \ldots, m, \quad Y_{l}(g)=\left(L_{g}\right)_{*}\left(Y_{l}\right), l=1, \ldots, k,
$$

where $\left(L_{g}\right)_{*}$ denotes the differential of $L_{g}$. The system $X$ defines a basis for the so-called horizontal sub-bundle $H G$ of the tangent bundle $T G$. For a given function $f: G \rightarrow \mathbb{R}$, the action of $X_{j}$ on $f$ is specified by the equation

$$
X_{j} f(g)=\lim _{t \rightarrow 0} \frac{f\left(g \exp \left(t X_{j}\right)\right)-f(g)}{t}=\left.\frac{d}{d t} f\left(g \exp \left(t X_{j}\right)\right)\right|_{t=0} .
$$


A similar formula holds for any left-invariant vector field. We indicate with

$x_{j}(g)=<\xi_{1}(g), X_{j}>, \quad j=1, \ldots, m, \quad y_{s}(g)=<\xi_{2}(g), Y_{s}>, \quad s=1, \ldots, k$.

the projections of the exponential coordinates of $g$ onto $V_{1}$ and $V_{2}$. Letting $x(g)=\left(x_{1}(g), \ldots, x_{m}(g)\right), y(g)=\left(y_{1}(g), \ldots, y_{k}(g)\right)$, we will often identify $g \in \boldsymbol{G}$ with its exponential coordinates

$$
g=(x(g), y(g), \ldots),
$$

where the dots indicate the $(N-(m+k))$-dimensional vector

$$
\left(x_{3,1}(g), \ldots, x_{3, m_{3}}(g), \ldots, x_{r, 1}(g), \ldots, x_{r, m_{r}}(g)\right) .
$$

When $\boldsymbol{G}$ is a group of step 2, then (2.6) simply becomes $g=(x(g), y(g))$. Such identification of $\boldsymbol{G}$ with its Lie algebra is justified by the BakerCampbell-Hausdorff formula, see, e.g., [V]

$$
\exp Z \exp Z^{\prime}=\exp \left(Z+Z^{\prime}+\frac{1}{2}\left[Z, Z^{\prime}\right]+\ldots\right) \quad Z, Z^{\prime} \in \mathfrak{g},
$$

where the dots indicate a finite linear combination of terms containing commutators of order two and higher. For $Z \in \mathfrak{g}$ consider the map $\theta_{Z}: \mathfrak{g} \rightarrow \mathfrak{g}$ given by

$$
\theta_{Z}\left(Z^{\prime}\right)=Z+Z^{\prime}+\frac{1}{2}\left[Z, Z^{\prime}\right]+\ldots
$$

where the right-hand side is given by the Baker-Campbell-Hausdorff sum in (2.7). If we endow the Lie algebra $\mathfrak{g}$ with the polynomial group law

$$
Z \circ Z^{\prime}=\theta_{Z}\left(Z^{\prime}\right)
$$

then we can identify the group $\boldsymbol{G}$ with $\mathfrak{g}$, via the exponential coordinates.

A Carnot group of step $r$ is naturally equipped with a family of nonisotropic dilations defined by

$$
\delta_{\lambda}(g)=\exp \circ \Delta_{\lambda} \circ \exp ^{-1}(g), \quad g \in \boldsymbol{G},
$$

where $\exp : \mathfrak{g} \rightarrow G$ is the exponential map and $\Delta_{\lambda}: \mathfrak{g} \rightarrow \mathfrak{g}$ is defined by $\Delta_{\lambda}\left(X_{1}+\ldots+X_{r}\right)=\lambda X_{1}+\ldots+\lambda^{r} X_{r}$.

The most basic partial differential operator in a Carnot group is the $s u b$ Laplacian associated with $X$ is the second-order partial differential operator on $\boldsymbol{G}$ given by

$$
\mathcal{L}=\sum_{j=1}^{m} X_{j}^{2} .
$$


By the assumption on the Lie algebra one immediately sees that the system $X$ satisfies the finite rank condition in Hörmander's theorem [H1], therefore the operator $\mathcal{L}$ is hypoelliptic. However, when $r>1$ it fails to be elliptic, and the loss of regularity is measured by the step $r$ of the stratification of $\mathfrak{g}$.

For a function $u$ on $\boldsymbol{G}$ we let

$$
X u=X_{1} u X_{1}+\ldots+X_{m} u X_{m},
$$

and set $|X u|=\left(\sum_{j=1}^{m}\left(X_{j} u\right)^{2}\right)^{1 / 2}$. We denote by $d g$ the push-forward of Lebesgue measure on $\mathfrak{g}$ via the exponential map. Such $d g$ defines a biinvariant Haar measure on $\boldsymbol{G}$. One has $d\left(g \circ \delta_{\lambda}\right)=\lambda^{Q} d g$, so that the number

$$
Q=m_{1}+2 m_{2}+\ldots+r m_{r}
$$

plays the role of a dimension with respect to the group dilations. For this reason $Q$ is called the homogeneous dimension of $\boldsymbol{G}$. Such number is larger than the topological dimension $N$ of $\boldsymbol{G}$ defined above.

The Euclidean distance to the origin $|\cdot|$ on $\mathfrak{g}$ induces a homogeneous pseudo-norm $|\cdot|_{\mathfrak{g}}$ on $\mathfrak{g}$ and (via the exponential map) one on the group $\boldsymbol{G}$ in the following way (see [F2], [FS]). For $\xi \in \mathfrak{g}$, with $\xi=\xi_{1}+\ldots+\xi_{r}, \xi_{i} \in V_{i}$, we let

$$
|\xi|_{\mathfrak{g}}=\left(\sum_{i=1}^{r}\left|\xi_{i}\right|^{2 r ! / i}\right)^{2 r !},
$$

and then define a pseudo-norm on $\boldsymbol{G}$ by the equation

$$
N(g)=N_{\boldsymbol{G}}(g)=|\xi|_{\mathfrak{g}}, \quad \text { if } g=\exp \xi .
$$

The function $N$ is usually referred to as the non-isotropic gauge. It defines a pseudo-distance on $\boldsymbol{G}$

$$
d\left(g, g^{\prime}\right)=N\left(g^{-1} g^{\prime}\right) .
$$

This is called the gauge pseudo-distance, and it is equivalent to the Carnot-Carathéodory distance $\rho(\cdot, \cdot)$ generated by the system $X$, i.e., there exists a constant $C=C(\boldsymbol{G})>0$ such that

$$
C \rho\left(g, g^{\prime}\right) \leq d\left(g, g^{\prime}\right) \leq C^{-1} \rho\left(g, g^{\prime}\right), \quad g, g^{\prime} \in \boldsymbol{G},
$$

see [Be]. If $B(g, R)=\left\{g^{\prime} \in \boldsymbol{G} \mid d\left(g, g^{\prime}\right)<R\right\}$, then by left-translation and dilation it is easy to see that the Haar measure of $B(g, R)$ is

$$
|B(g, R)|=\omega R^{Q},
$$


where $\omega=|B(e, 1)|$, with $e$ indicating the group identity. A similar translation invariance holds for the metric balls $B_{\rho}(g, R)$.

The Heisenberg group. A model of Carnot group of paramount importance is the Heisenberg group $\mathbb{H}^{n}$, see $[\mathrm{S}],[\mathrm{G}]$. The underlying manifold of this Lie group is simply $\mathbb{R}^{2 n+1}$, with the non-commutative group law (2.15)

$g g^{\prime}=(x, y, t)\left(x^{\prime}, y^{\prime}, t^{\prime}\right)=\left(x+x^{\prime}, y+y^{\prime}, t+t^{\prime}+2\left(<x^{\prime}, y>-<x, y^{\prime}>\right)\right)$,

where we have let $x, x^{\prime}, y, y^{\prime} \in \mathbb{R}^{n}, t, t^{\prime} \in \mathbb{R}$. Let $\left(L_{g}\right)_{*}$ be the differential of the left-translation (2.15). A simple computation shows that

$$
\begin{aligned}
& \left(L_{g}\right)_{*}\left(\frac{\partial}{\partial x_{j}}\right) \stackrel{\text { def }}{=} X_{j}=\frac{\partial}{\partial x_{j}}+2 y_{j} \frac{\partial}{\partial t}, \quad j=1, \ldots, n, \\
& \left(L_{g}\right)_{*}\left(\frac{\partial}{\partial y_{j}}\right) \stackrel{\text { def }}{=} X_{n+j}=\frac{\partial}{\partial y_{j}}-2 x_{j} \frac{\partial}{\partial t}, \quad j=1, \ldots, n, \\
& \left(L_{g}\right)_{*}\left(\frac{\partial}{\partial t}\right) \stackrel{\text { def }}{=} X_{2 n+1}=\frac{\partial}{\partial t}
\end{aligned}
$$

We note that the only non-trivial commutator is

$$
\left[X_{j}, X_{n+k}\right]=-4 X_{2 n+1} \delta_{j k}, \quad j, k=1, \ldots, n,
$$

therefore the vector fields $\left\{X_{1}, \ldots, X_{2 n}\right\}$ constitute a basis of the Lie algebra $\mathfrak{h}_{n}=\mathbb{R}^{2 n+1}=V_{1} \oplus V_{2}$, where $V_{1}=\mathbb{R}^{2 n} \times\{0\}_{t}, V_{2}=\{0\}_{(x, y)} \times \mathbb{R}$. The non-isotropic group dilations are therefore

$$
\delta_{\lambda}(g)=\left(\lambda x, \lambda y, \lambda^{2} t\right) .
$$

The associated homogeneous dimension is $Q=2 n+2$, and the gauge is given by

$$
N(g)=\left(\left(|x|^{2}+|y|^{2}\right)^{2}+t^{2}\right)^{1 / 4} .
$$

We notice that the sub-Laplacian associated with the basis $\left\{X_{1}, \ldots, X_{2 n}\right\}$ is

$$
\mathcal{L}=\sum_{j=1}^{2 n} X_{j}^{2}=\Delta_{x, y}+4\left(|x|^{2}+|y|^{2}\right) \frac{\partial^{2}}{\partial t^{2}}+4 \frac{\partial}{\partial t} \sum_{j=1}^{n}\left\{y_{j} \frac{\partial}{\partial x_{j}}-x_{j} \frac{\partial}{\partial y_{j}}\right\}
$$

which coincides with the real part of the complex Kohn Laplacian, see [S].

Returning to general Carnot groups, we recall that for a given open set $\Omega \subset \boldsymbol{G}$, the classes $\Gamma^{1}(\Omega)$ (respectively, $\Gamma^{2}(\Omega)$ ) introduced by Folland and Stein [FS], [F2], represent the collection of all functions $u: \Omega \rightarrow \mathbb{R}$ such that the derivatives $X_{\alpha} u$ (respectively, $X_{\alpha} X_{\alpha^{\prime}} u$ ), $\alpha, \alpha^{\prime}=1, \ldots, m$, exist and are continuous functions in $\Omega$. We also need the definition of Lipschitz function. 
Definition 2.1. Let $\Omega \subset G$ be an open set. A function $u: \Omega \rightarrow \mathbb{R}$ is said to belong to the class $\Gamma^{0,1}(\Omega)$ if there exists a positive constant $L>0$ such that

$$
\left|u(g)-u\left(g^{\prime}\right)\right| \leq L d\left(g, g^{\prime}\right), \quad g, g^{\prime} \in \Omega .
$$

The meaning of the symbol $\Gamma_{\text {loc }}^{0,1}(\Omega)$ is the obvious one, i.e., $u \in \Gamma_{\text {loc }}^{0,1}(\Omega)$ if for every $\omega \subset \subset \Omega$ one has $u \in \Gamma^{0,1}(\omega)$. If $F \subset \boldsymbol{G}$ denotes a closed set, by $u \in \Gamma^{0,1}(F)$ we mean that $u$ coincides on the set $F$ with a function $g \in \Gamma^{0,1}(\Omega)$, where $\Omega$ is a bounded open set containing $F$. The condition (2.19) in the Definition 2.1 measures the smoothness of $u$ in the intrinsic pseudo-metric $d\left(g, g^{\prime}\right)$ on $\boldsymbol{G}$ associated with the system $X=\left\{X_{1}, \ldots, X_{m}\right\}$ (of course, using the distance $\rho\left(g, g^{\prime}\right)$, instead of $d\left(g, g^{\prime}\right)$, in view of $(2.13)$ would produce the same functional classes). The corresponding regularity of $u$ with respect to the underlying Riemannian metric $d_{R}\left(g, g^{\prime}\right)$ of $\boldsymbol{G}$ is much lower (depending on the step $r$ of the group), since one has for $\omega \subset \subset \boldsymbol{G}$, for some constant $C=C(\boldsymbol{G}, \omega)>0$

$$
C d_{R}\left(g, g^{\prime}\right) \leq d\left(g, g^{\prime}\right) \leq C^{-1} d_{R}\left(g, g^{\prime}\right)^{1 / r} .
$$

In his foundational paper $[\mathrm{P}]$ Pansu proved the following remarkable result of Rademacher type for maps $u: G \rightarrow \boldsymbol{G}^{\prime}$ between two Carnot groups.

Theorem 2.2. Let $\boldsymbol{G}, \boldsymbol{G}^{\prime}$ be two Carnot groups, and consider open sets $\Omega \subset \boldsymbol{G}, \Omega^{\prime} \subset \boldsymbol{G}^{\prime}$. If $u: \Omega \rightarrow \Omega^{\prime}$ is a Lipschitz map with respect to the relative intrinsic metrics, i.e., there exists a constant $L>0$ such that

$$
d^{\prime}\left(u\left(g_{1}\right), u\left(g_{2}\right)\right) \leq L d\left(g_{1}, g_{2}\right), \quad g_{1}, g_{2} \in \Omega,
$$

then the differential of $u, D u(g)$, exists for $d g$-a.e. point $g \in \Omega$.

The notion of differential of a map between Carnot groups was introduced by Pansu himself. One says that $u$ is Pansu differentiable at $g \in \boldsymbol{G}$ if the maps

$$
u_{\lambda}=\delta_{\lambda^{-1}}^{\prime} \circ L_{u(g)^{-1}}^{\prime} \circ u \circ L_{g} \circ \delta_{\lambda}
$$

converge locally uniformly to a homomorphism $D u(g): \boldsymbol{G} \rightarrow \boldsymbol{G}^{\prime}$, as $\lambda \rightarrow 0$. Here, $L_{g}(h)=g h$ denotes the operator of left-translation on $\boldsymbol{G}$, whereas we have denoted by $L_{g^{\prime}}^{\prime}=g^{\prime} h^{\prime}$ the analogous operator on $G^{\prime}$. Similarly, $\delta_{\lambda}$ and $\delta_{\lambda}^{\prime}$ respectively denote the dilations on $\boldsymbol{G}$ and $\boldsymbol{G}^{\prime}$. 
We note explicitly that when the target group is $G^{\prime}=(\mathbb{R},+)$, then the definition (2.20) of Pansu differential of a function $u: G \rightarrow \mathbb{R}$ becomes

$$
D u(g)(h)=\lim _{\lambda \rightarrow 0^{+}} \frac{u\left(g \delta_{\lambda}(h)\right)-u(g)}{\lambda},
$$

the limit being assumed locally uniform in $h \in \boldsymbol{G}$. In the next proposition we give a characterization of formula (2.21) in the case in which $u$ is sufficiently smooth.

Proposition 2.3. Let $\boldsymbol{G}$ be a Carnot group of step $r$. For $u \in \Gamma^{1}(\boldsymbol{G})$ the Pansu differential of $u$ is given by the formula

$$
D u(g)(h)=\sum_{j=1}^{m} x_{j}(h) X_{j} u(g)=<\xi_{1}(h), X u(g)>, \quad g, h \in \boldsymbol{G}
$$

When $u \in C^{1}(\boldsymbol{G})$ the proof of the above result can be found in [DGS], or also in $[\mathrm{MSC}]$. The passage from $C^{1}(\boldsymbol{G})$ to $\Gamma^{1}(\boldsymbol{G})$ is easily justified by mollification with group convolution, see equation (3.5) for the relevant definition.

We will need the following quantitative version of Theorem 2.2.

Theorem 2.4. Let $\Omega \subset \boldsymbol{G}$ be a bounded open set. If for $u \in \Gamma^{0,1}(\Omega)$ and (2.19) holds, then the distributional derivatives of $u$ along the vector fields $X_{1}, \ldots, X_{m}$ belong to $L^{\infty}(\Omega)$, and moreover there exists $C=C(\boldsymbol{G})>0$ such that

$$
\left\|X_{\alpha} u\right\|_{L^{\infty}(\Omega)} \leq C L \quad \alpha=1, \ldots, m .
$$

Theorem 2.4 is just a special case of a result which in [GN2] was proved for a general family of Lipschitz vector fields in $\mathbb{R}^{n}$, see also [FSS], where the same result was independently obtained. We will also need the following converse to Theorem 2.4 which is also a special case of a general result in [GN2]. Given a measurable set $E \subset \boldsymbol{G}$, we say that a function $u \in L^{p}(E)$ belongs to $\mathcal{L}^{1, p}(E), 1 \leq p \leq \infty$, if the distributional derivatives $X_{\alpha} u \in$ $L^{p}(E)$, for $\alpha=1, \ldots m$. The space $\mathcal{L}^{1, p}(E)$ becomes a Banach space with the norm

$$
\|u\|_{\mathcal{L}^{1, p}(E)}=\|u\|_{L^{p}(E)}+\sum_{\alpha=1}^{m}\left\|X_{\alpha} u\right\|_{L^{p}(E)} .
$$


Theorem 2.5. Given a Carnot group $\boldsymbol{G}$, let $u \in \mathcal{L}^{1, \infty}\left(B\left(g_{o}, 3 R\right)\right)$, with $g_{o} \in \boldsymbol{G}$ and $R>0$, then $u$ can be modified on a set of $d g$-measure zero in $\bar{B}=\bar{B}\left(g_{o}, R\right)$, so as to satisfy for every $g, g^{\prime} \in \bar{B}\left(g_{o}, R\right)$

$$
\left|u(g)-u\left(g^{\prime}\right)\right| \leq C d\left(g, g^{\prime}\right)\|u\|_{\mathcal{L}^{1, \infty}\left(B\left(g_{o}, 3 R\right)\right)},
$$

for some $C=C(\boldsymbol{G})>0$. If, furthermore, $u \in C^{\infty}\left(B\left(g_{o}, 3 R\right)\right)$, then in the right hand side of the previous inequality one can replace the term $\|u\|_{\mathcal{L}^{1, \infty}\left(B\left(g_{o}, 3 R\right)\right)}$ with $\|X u\|_{L^{\infty}\left(B\left(g_{o}, 3 R\right)\right)}$.

Combining Theorems 2.5 and 2.4 we obtain a characterization of the Lipschitz class $\Gamma_{l o c}^{0,1}(\boldsymbol{G})$ as the space $\mathcal{L}_{\text {loc }}^{1, \infty}(\boldsymbol{G})$.

\section{Strongly convex functions: A notion to abandon.}

The notion of Pansu differential expressed by equation (2.21), and its connection with the horizontal gradient established by Proposition 2.3, suggests a first notion of convexity in a Carnot group, which we call strong $H$-convexity. At the beginning of Section 4 we will see that such concept is too restrictive for the development of a satisfactory theory, and has to be modified. Another basic reason to renounce the notion of strong $H$-convexity lies in the fact that the gauge balls in the Heisenberg group are not strongly $H$-convex, see Remark 7.5 in Section 7. But...we are getting ahead of our story. It is time to introduce a basic definition.

Definition 3.1. Given two points $g, g^{\prime} \in \boldsymbol{G}$, for $\lambda \in[0,1]$ we will denote

$$
g_{\lambda}=g_{\lambda}\left(g ; g^{\prime}\right)=\stackrel{\text { def }}{=} g \delta_{\lambda}\left(g^{-1} g^{\prime}\right)
$$

the twisted "convex combination" of $g$ and $g^{\prime}$ based at $g$.

We note that unlike the standard convex combination in a vector space, in Definition 3.1 the base point $g$ plays a privileged role. This unavoidable drawback is connected with the non-commutative structure of the ambient spaces, and it is a source of complications, such as for instance lack of a well-behaved Jensen inequality. Different, more symmetric, "convex combinations" could be proposed. For instance, one might take $g_{\lambda}=\delta_{1-\lambda} g \delta_{\lambda} g^{\prime}$, but a careful analysis reveals the unsuitability of the latter.

Before proceeding we note explicitly that since we are using the pseudodistance generated by the gauge (2.12) we have for every $\lambda>0$

$$
\lambda=\frac{d\left(g_{\lambda}, g\right)}{d\left(g^{\prime}, g\right)} .
$$


This is easily recognized as follows

$$
d\left(g_{\lambda}, g\right)=N\left(g^{-1} g_{\lambda}\right)=N\left(\delta_{\lambda}\left(g^{-1} g^{\prime}\right)\right)=\lambda N\left(g^{-1} g^{\prime}\right)=\lambda d\left(g^{\prime}, g\right) .
$$

Definition 3.2. A function $u: G \rightarrow(-\infty, \infty]$ is called strongly $H$-convex if it is proper, which means that $\{g \in \boldsymbol{G} \mid u(g)=\infty\} \neq \boldsymbol{G}$, and if for every $g, g^{\prime} \in \boldsymbol{G}$ one has

$$
u\left(g_{\lambda}\right) \leq u(g)+\lambda\left(u\left(g^{\prime}\right)-u(g)\right),
$$

for every $0 \leq \lambda \leq 1$. The effective domain of $u$ is the set

$$
\operatorname{dom}_{H} u=\{g \in \boldsymbol{G} \mid u(g)<\infty\} .
$$

We denote by $\mathcal{C}_{H}^{s}(\boldsymbol{G})$ the class of all strongly $H$-convex functions on $\boldsymbol{G}$.

We note right-away that in the abelian case $\boldsymbol{G}=\mathbb{R}^{n}$, Definition 3.2 coincides with the classical notion of convexity. The next propositions are direct consequences of Definition 3.2.

Proposition 3.3. For every $h \in \boldsymbol{G}$ the left-translated $L_{h} u(g)=u(h g)$ of a strongly $H$-convex function $u$ is also strongly $H$-convex.

Proof. For every $g, g^{\prime} \in \boldsymbol{G}$, and any $0 \leq \lambda \leq 1$ one has $L_{h} u\left(g \delta_{\lambda}\left(g^{-1} g^{\prime}\right)\right)=u\left(h g \delta_{\lambda}\left((h g)^{-1}\left(h g^{\prime}\right)\right)\right) \leq L_{h} u(g)+\lambda\left(L_{h} u\left(g^{\prime}\right)-L_{h} u(g)\right)$.

Proposition 3.4. Let $\left\{u_{\alpha}\right\}_{\alpha \in \mathcal{A}}$ be an arbitrary family of strongly $H$-convex functions. If $\sup _{\alpha \in \mathcal{A}} u_{\alpha}$ is proper, then it belongs to $\mathcal{C}_{H}^{s}(\boldsymbol{G})$. If $u, v \in \mathcal{C}_{H}^{s}(\boldsymbol{G})$ , and $c \geq 0$, then $u+v$ and $c u$ are in $\mathcal{C}_{H}^{s}(\boldsymbol{G})$ if they are proper.

Proposition 3.5. Let $h: \mathbb{R} \rightarrow \mathbb{R}$ be convex and non-decreasing, then for every strongly $H$-convex function $u: G \rightarrow \mathbb{R}$, the function $h \circ u$ is strongly $H$-convex.

The following definition, and the subsequent propositions, provide us with a reasonably rich supply of strongly $H$-convex functions.

Definition 3.6. A function $A: G \rightarrow \mathbb{R}$ is called $H$-affine if there exists $p=\sum_{\alpha=1}^{m} p_{\alpha} X_{\alpha} \in V_{1}$, and $\beta \in \mathbb{R}$ such that

$$
A(g)=\sum_{j=1}^{m} p_{j} x_{j}(g)+\beta=<p, \xi_{1}(g)>+\beta .
$$


Proposition 3.7. Every $H$-affine function is strongly $H$-convex.

Proof. Suppose that $A$ is of the form (3.3), then

$$
A\left(g \delta_{\lambda}\left(g^{-1} g^{\prime}\right)\right)=<p, \xi_{1}\left(g \delta_{\lambda}\left(g^{-1} g^{\prime}\right)\right)>+\beta .
$$

We now observe that the formula of Baker-Campbell-Hausdorff (2.7) gives

$$
\xi_{1}\left(g \delta_{\lambda}\left(g^{-1} g^{\prime}\right)\right)=\xi_{1}(g)+\lambda\left(\xi_{1}\left(g^{\prime}\right)-\xi_{1}(g)\right),
$$

therefore

$$
\begin{aligned}
A\left(g \delta_{\lambda}\left(g^{-1} g^{\prime}\right)\right) & =<p, \xi_{1}(g)+\lambda\left(\xi_{1}\left(g^{\prime}\right)-\xi_{1}(g)\right)>+\beta \\
& =A(g)+\lambda\left(A\left(g^{\prime}\right)-A(g)\right) .
\end{aligned}
$$

Proposition 3.8. Let $\phi: \mathbb{R}^{m} \rightarrow(-\infty, \infty]$, then the function $u: G \rightarrow$ $(-\infty, \infty]$ defined by

$$
u(g) \stackrel{\text { def }}{=} \phi(x(g)),
$$

is strongly $H$-convex if and only if $\phi$ is a standard convex function.

Proof. From (3.4) we obtain

$$
u\left(g \delta_{\lambda}\left(g^{-1} g^{\prime}\right)\right)=\phi\left(x(g)+\lambda\left(x\left(g^{\prime}\right)-x(g)\right)\right) .
$$

The conclusion easily follows from this formula.

Convexity and convolution. If $u$ and $v$ are two measurable functions on $\boldsymbol{G}$, their convolution is defined by

$$
u \star v(g)=\int_{\boldsymbol{G}} u(h) v\left(h^{-1} g\right) d h=\int_{\boldsymbol{G}} u\left(g h^{-1}\right) v(h) d h .
$$

Since convolution is a smoothing process it is important to know that a notion of convexity is preserved under such operation. This follows from Proposition 3.3.

Proposition 3.9. Let $u: \boldsymbol{G} \rightarrow \mathbb{R}$ be strongly $H$-convex and $K \in L^{1}(\boldsymbol{G})$, with $K \geq 0$, then $K \star u$ is also strongly $H$-convex if it is proper. 
Proof. For $0 \leq \lambda \leq 1$ one has from (3.5) and from Definition 3.2

$$
\begin{aligned}
K \star u\left(g \delta_{\lambda}\left(g^{-1} g^{\prime}\right)\right) & =\int_{\boldsymbol{G}} K(h) u\left(h^{-1} g \delta_{\lambda}\left(g^{-1} g^{\prime}\right) d h\right. \\
& =\int_{\boldsymbol{G}} K(h) u\left(h^{-1} g \delta_{\lambda}\left(\left(h^{-1} g\right)^{-1}\left(h^{-1} g^{\prime}\right)\right) d h\right. \\
& \leq \int_{\boldsymbol{G}} K(h)\left\{u\left(h^{-1} g\right)+\lambda\left(u\left(h^{-1} g^{\prime}\right)-u\left(h^{-1} g\right)\right)\right\} d h \\
& =K \star u(g)+\lambda\left(K \star u\left(g^{\prime}\right)-K \star u(g)\right) .
\end{aligned}
$$

\section{Mildly convex functions: The appearance of horizontal planes.}

Although the notion of strongly $H$-convex function produces some interesting results, a careful analysis of it reveals its restrictive nature. In the Abelian case of $\mathbb{R}^{n}$ the most important prototype of convex functions are the quadratic homogeneous polynomials $P(x)=\sum_{i, j=1}^{n} \alpha_{i j} x_{i} x_{j}$, with $\alpha_{i j}=\alpha_{j i}$, and $\left(\alpha_{i j}\right)_{i, j=1, \ldots, n} \geq 0$. If we consider for instance the Heisenberg group $\mathbb{H}^{1}$, with the parabolic dilations (2.17), then the appropriate homogeneous polynomials of non-isotropic degree 2 are of the form

$$
P(x, y, t)=\alpha x^{2}+2 \beta x y+\gamma y^{2}+\delta t,
$$

where the matrix $\left(\begin{array}{ll}\alpha & \beta \\ \beta & \gamma\end{array}\right)$ is assumed $\geq 0$. Since, thanks to Proposition 3.8 we know that $Q(x, y, t)=\alpha x^{2}+2 \beta x y+\gamma y^{2}$ is strongly $H$-convex, it is natural to ask whether such is also the function $u(x, y, t)=t$. This question is further motivated by the fact that such "quadratic function" is also $\mathcal{L}$-harmonic on $\mathbb{H}^{n}$, i.e., it is an entire solution of the Kohn sub-Laplacian (2.18). To verify the strong $H$-convexity of $u$, let $g=(x, y, t), g^{\prime}=\left(x^{\prime}, y^{\prime}, t^{\prime}\right)$ be two points in $\mathbb{H}^{1}$, and consider the point defined by (3.1)

$$
\begin{aligned}
g_{\lambda}= & \left(x+\lambda\left(x^{\prime}-x\right), y+\lambda\left(y^{\prime}-y\right),\right. \\
& \left.t+2 \lambda\left(x^{\prime} y-x y^{\prime}\right)+\lambda^{2}\left(t^{\prime}-t+2 x y^{\prime}-2 x^{\prime} y\right)\right) .
\end{aligned}
$$

We have from (4.1)

$$
u\left(g_{\lambda}\right)=t+2 \lambda\left(x^{\prime} y-x y^{\prime}\right)+\lambda^{2}\left(t^{\prime}-t+2 x y^{\prime}-2 x^{\prime} y\right)
$$


so that Definition 3.2 now reads

$$
t+2 \lambda\left(x^{\prime} y-x y^{\prime}\right)+\lambda^{2}\left(t^{\prime}-t+2 x y^{\prime}-2 x^{\prime} y\right) \leq t+\lambda\left(t^{\prime}-t\right)
$$

One readily sees that the latter inequality is equivalent to

$$
(1-\lambda)\left(2 x y^{\prime}-2 x^{\prime} y\right) \leq(1-\lambda)\left(t^{\prime}-t\right)
$$

which in turn holds if and only if

$$
t^{\prime} \geq t+2 x y^{\prime}-2 x^{\prime} y .
$$

We conclude from (4.2) that the function $u(x, y, t)=t$ is not strongly $H$-convex in $\mathbb{H}^{1}$. This important example prompts us to relax Definition 3.2. First, we introduce a definition which plays a pervasive role in this paper.

Definition 4.1. Let $\boldsymbol{G}$ be a Carnot group with Lie algebra $\mathfrak{g}=V_{1} \oplus \ldots \oplus V_{r}$, and let $m=\operatorname{dim} V_{1}$. Given a point $g_{o} \in \boldsymbol{G}$ we define the horizontal plane through $g_{o}$ as the $m$-dimensional embedded submanifold of $\boldsymbol{G}$ given by

$$
H_{g_{o}}=L_{g_{o}}\left(\exp \left(V_{1} \times\{0\}\right)\right)
$$

where 0 denotes the $N-m$-dimensional zero vector in $\mathfrak{g}$, with $N=\operatorname{dim} V_{1}+$ $\ldots+\operatorname{dim} V_{r}$. In particular, we see that

$$
H_{e}=\exp \left(V_{1} \times\{0\}\right), \quad \text { and } \quad H_{g_{o}}=L_{g_{o}}\left(H_{e}\right) .
$$

We remark explicitly that $H_{e}$ is not a subgroup of $\boldsymbol{G}$ since it is not true, in general, that $g, g^{\prime} \in H_{e}$ implies $g g^{\prime} \in H_{e}$. For instance, in the Heisenberg group $\mathbb{H}^{n}$ we have from $(2.15)$

$$
g g^{\prime}=(x, y, 0)\left(x^{\prime}, y^{\prime}, 0\right)=\left(x+x^{\prime}, y+y^{\prime}, 2\left(<x^{\prime}, y>-<x, y^{\prime}>\right)\right),
$$

so that $g g^{\prime} \in H_{e}$ only when the two vectors $\left(x^{\prime}, y^{\prime}\right),(y,-x) \in \mathbb{R}^{2 n}$ are orthogonal. However, it is true that $g \in H_{e}$ if and only if $g^{-1} \in H_{e}$, and $\delta_{\lambda} g \in H_{e}$ for $\lambda>0$. From Definition 4.1 we immediately obtain the following elementary, yet important, result.

Proposition 4.2. A point $g \in \boldsymbol{G}$ belongs to $H_{g_{o}}$ if and only if $g_{o}^{-1} g \in H_{e}$. Moreover, $g^{\prime} \in H_{g}$ if and only if $g \in H_{g^{\prime}}$. Finally, if $g^{\prime} \in H_{g}$, then one also has $g_{\lambda} \in H_{g}$ for every $\lambda \in[0,1]$. 
Proof. Only the second and third parts require a proof. If $g^{\prime} \in H_{g}$, then $g^{-1} g^{\prime} \in H_{e}$. If $\xi(g)=\xi_{1}(g)+\ldots+\xi_{r}(g)$ denote the exponential coordinates of $g$, we see that the condition $g^{-1} g^{\prime} \in H_{e}$ translates into

$$
\xi_{2}\left(g^{-1} g^{\prime}\right)=0, \ldots, \xi_{r}\left(g^{-1} g^{\prime}\right)=0 .
$$

These are the general equations of the horizontal plane $H_{g}$. Since by (2.7) we have $\xi\left(g^{-1}\right)=-\xi(g)$, we conclude that $g^{-1} g^{\prime} \in H_{e}$ if and only if $\left(g^{\prime}\right)^{-1} g \in H_{e}$, which is equivalent to $g \in H_{g^{\prime}}$.

For the third part we observe that for every $\lambda \in[0,1]$ one has $g_{\lambda} \in H_{g}$ if and only if $g^{-1} g_{\lambda}=\delta_{\lambda}\left(g^{-1} g^{\prime}\right) \in H_{e}$. Since for any point $h \in \boldsymbol{G}$ we have $\delta_{\lambda} h \in H_{e}$ if and only if $h \in H_{e}$, we conclude that $g_{\lambda} \in H_{g}$ is equivalent to $g^{-1} g^{\prime} \in H_{e}$, i.e., $g^{\prime} \in H_{g}$. This completes the proof.

In the sequel it will be convenient to have for a given $g \in \boldsymbol{G}$ an explicit description of the horizontal plane $H_{g}$ given by $N-m$ polynomial equations. Such equations are given by the $r-1$ vector equations (4.3). In the next proposition we calculate such equations in the interesting setting of groups of step 2. For a group of higher step one has similar, but more complicated formulas.

Given a Carnot group $\boldsymbol{G}$ of step 2, with Lie algebra $\mathfrak{g}=V_{1} \oplus V_{2}$, consider the mapping $J: V_{2} \rightarrow \operatorname{End}\left(V_{1}\right)$ defined by

$$
<J(\eta) \xi^{\prime}, \xi^{\prime \prime}>=<\left[\xi^{\prime}, \xi^{\prime \prime}\right], \eta>, \quad \eta \in V_{2}, \quad \xi^{\prime}, \xi^{\prime \prime} \in V_{1} .
$$

The algebraic properties of the mapping $J$ have important repercussions on the geometric and analytic properties of Carnot groups of step 2. An immediate consequence of the definition of $J$ is that

$$
<J(\eta) \xi, \xi>=0, \quad \text { for every } \quad \eta \in V_{2}, \xi \in V_{1} .
$$

Proposition 4.3. Let $\boldsymbol{G}$ be a Carnot group of step 2, then for any given $g_{o} \in \boldsymbol{G}$ the horizontal plane passing through $g_{o}$ is the collection of all points $g \in \boldsymbol{G}$ whose exponential coordinates $(x(g), y(g))$ verify the $k$ linear equations

$$
y_{\beta}(g)=y_{\beta}\left(g_{o}\right)+\frac{1}{2} \sum_{\alpha, \alpha^{\prime}=1}^{m} b_{\alpha \alpha^{\prime}}^{\beta} x_{\alpha}\left(g_{o}\right) x_{\alpha^{\prime}}(g), \quad \beta=1, \ldots, k,
$$

where $b_{\alpha \alpha^{\prime}}^{\beta}$ represent the group constants

$$
\left[X_{\alpha}, X_{\alpha^{\prime}}\right]=\sum_{\beta=1}^{k} b_{\alpha \alpha^{\prime}}^{\beta} Y_{\beta}, \quad \alpha, \alpha^{\prime}=1, \ldots, m .
$$


Proof. If we let $g_{o}=\exp \left(\xi\left(g_{o}\right)\right), g=\exp (\xi(g))$, keeping in mind that $g_{o}^{-1}=\exp \left(-\xi\left(g_{o}\right)\right)$, we have from $(2.7)$

$g_{o}^{-1} g=\exp \left(-\xi\left(g_{o}\right)\right) \exp (\xi(g))=\exp \left(-\xi\left(g_{o}\right)+\xi(g)-\frac{1}{2}\left[\xi\left(g_{o}\right), \xi(g)\right]\right)$.

Writing $\xi\left(g_{o}\right)=\xi_{1}\left(g_{o}\right)+\xi_{2}\left(g_{o}\right), \xi(g)=\xi_{1}(g)+\xi_{2}(g)$, we obtain from the latter equation

$$
\begin{aligned}
g_{o}^{-1} g & =\exp \left(-\xi\left(g_{o}\right)\right) \exp (\xi(g)) \\
& =\exp \left(-\xi_{1}\left(g_{o}\right)+\xi_{1}(g)-\xi_{2}\left(g_{o}\right)+\xi_{2}(g)-\frac{1}{2}\left[\xi_{1}\left(g_{o}\right), \xi_{1}(g)\right]\right) .
\end{aligned}
$$

This formula shows that

$$
\xi_{1}\left(g_{o}^{-1} g\right)=\xi_{1}(g)-\xi_{1}\left(g_{o}\right), \quad \xi_{2}\left(g_{o}^{-1} g\right)=\xi_{2}(g)-\xi_{2}\left(g_{o}\right)-\frac{1}{2}\left[\xi_{1}\left(g_{o}\right), \xi_{1}(g)\right] .
$$

Since $g \in H_{g_{o}}$ is equivalent to $g_{o}^{-1} g \in H_{e}$, the second equation in (4.6) gives

$$
\xi_{2}\left(g_{o}^{-1} g\right)=\xi_{2}(g)-\xi_{2}\left(g_{o}\right)-\frac{1}{2}\left[\xi_{1}\left(g_{o}\right), \xi_{1}(g)\right]=0 \in V_{2} .
$$

Keeping in mind that $\xi_{1}(g)=\sum_{\alpha=1}^{m} x_{\alpha}(g) X_{\alpha}, \xi_{2}(g)=\sum_{\beta=1}^{k} y_{\beta}(g) Y_{\beta}$, we conclude

$$
\xi_{2}(g)=\xi_{2}\left(g_{o}\right)+\frac{1}{2} \sum_{\alpha, \alpha^{\prime}=1}^{m} x_{\alpha}\left(g_{o}\right) x_{\alpha^{\prime}}(g)\left[X_{\alpha}, X_{\alpha^{\prime}}\right],
$$

which we can rewrite as follows

$$
\sum_{\beta=1}^{k} y_{\beta}(g) Y_{\beta}=\sum_{\beta=1}^{k} y_{\beta}\left(g_{o}\right) Y_{\beta}+\frac{1}{2} \sum_{\beta=1}^{k}\left(\sum_{\alpha, \alpha^{\prime}=1}^{m} b_{\alpha \alpha^{\prime}}^{\beta} x_{\alpha}\left(g_{o}\right) x_{\alpha^{\prime}}(g)\right) Y_{\beta} \text {. }
$$

This completes the proof.

The next proposition shows that, interestingly, when $g^{\prime} \in H_{g}$ then the twisted "convex combination" of $g$ and $g^{\prime}$ given by (3.1) coincides, at the level of the Lie algebra, with the standard Euclidean convex combination.

Proposition 4.4. Let $\boldsymbol{G}$ be a Carnot group. Given $g \in \boldsymbol{G}$, then for any $g^{\prime} \in H_{g}$ one has

$$
\xi\left(g_{\lambda}\right)=(1-\lambda) \xi(g)+\lambda \xi\left(g^{\prime}\right),
$$

for every $\lambda \in[0,1]$. 
Proof. We only present the proof for groups of step 2, the details are similar but more (tediously) complicated in the case of higher step. We have already observed in Proposition 4.2 that $g^{\prime} \in H_{g}$ if and only if $g_{\lambda} \in H_{g}$ for every $0 \leq \lambda \leq 1$. Proposition 4.3 gives

$$
y_{\beta}\left(g_{\lambda}\right)=y_{\beta}(g)+\frac{1}{2} \sum_{\alpha, \alpha^{\prime}=1}^{m} b_{\alpha \alpha^{\prime}}^{\beta} x_{\alpha}(g) x_{\alpha^{\prime}}\left(g_{\lambda}\right), \quad \beta=1, \ldots, k,
$$

Using (2.7) we easily find

$$
\xi_{1}\left(g_{\lambda}\right)=(1-\lambda) \xi_{1}(g)+\lambda \xi_{1}\left(g^{\prime}\right) .
$$

This gives for $\beta=1, \ldots, k$

$$
y_{\beta}\left(g_{\lambda}\right)=y_{\beta}(g)+\frac{1-\lambda}{2} \sum_{\alpha, \alpha^{\prime}=1}^{m} b_{\alpha \alpha^{\prime}}^{\beta} x_{\alpha}(g) x_{\alpha^{\prime}}(g)+\frac{\lambda}{2} \sum_{\alpha, \alpha^{\prime}=1}^{m} b_{\alpha \alpha^{\prime}}^{\beta} x_{\alpha}(g) x_{\alpha^{\prime}}\left(g^{\prime}\right) \text {. }
$$

Since

$$
b_{\alpha \alpha^{\prime}}^{\beta}=<\left[X_{\alpha}, X_{\alpha^{\prime}}\right], Y_{\beta}>=<J\left(Y_{\beta}\right) X_{\alpha}, X_{\alpha^{\prime}}>,
$$

we easily conclude that

$$
\begin{aligned}
& \sum_{\alpha, \alpha^{\prime}=1}^{m} b_{\alpha \alpha^{\prime}}^{\beta} x_{\alpha}(g) x_{\alpha^{\prime}}(g) \\
= & \sum_{\alpha, \alpha^{\prime}=1}^{m}<\xi_{1}(g), X_{\alpha}><\xi_{1}(g), X_{\alpha^{\prime}}><J\left(Y_{\beta}\right) X_{\alpha}, X_{\alpha^{\prime}}> \\
= & \sum_{\alpha=1}^{m}<J\left(Y_{\beta}\right) X_{\alpha}, \xi_{1}(g)><\xi_{1}(g), X_{\alpha}> \\
= & \sum_{\alpha=1}^{m}<J\left(Y_{\beta}\right)^{T} \xi_{1}(g), X_{\alpha}><\xi_{1}(g), X_{\alpha}> \\
= & -\sum_{\alpha=1}^{m}<J\left(Y_{\beta}\right) \xi_{1}(g), X_{\alpha}><\xi_{1}(g), X_{\alpha}> \\
= & -<J\left(Y_{\beta}\right) \xi_{1}(g), \xi_{1}(g)>=0,
\end{aligned}
$$

where in the latter equality we have used (4.5). Substitution of (4.9) in (4.8) gives

$$
y_{\beta}\left(g_{\lambda}\right)=y_{\beta}(g)+\frac{\lambda}{2} \sum_{\alpha, \alpha^{\prime}=1}^{m} b_{\alpha \alpha^{\prime}}^{\beta} x_{\alpha}(g) x_{\alpha^{\prime}}\left(g^{\prime}\right)
$$


Recalling that from Proposition 4.3 we have

$$
\frac{1}{2} \sum_{\alpha, \alpha^{\prime}=1}^{m} b_{\alpha \alpha^{\prime}}^{\beta} x_{\alpha}(g) x_{\alpha^{\prime}}\left(g^{\prime}\right)=y_{\beta}\left(g^{\prime}\right)-y_{\beta}(g),
$$

we conclude from (4.10) that

$$
\xi_{2}\left(g_{\lambda}\right)=(1-\lambda) \xi_{2}(g)+\lambda \xi_{2}\left(g^{\prime}\right) .
$$

Since $\xi(g)=\xi_{1}(g)+\xi_{2}(g)$, the latter equation and (4.7) imply the conclusion.

It is easy to recognize that, when $\boldsymbol{G}$ is the Heisenberg group $\mathbb{H}^{n}$, Definition 4.1 gives the following.

Proposition 4.5. Consider a point $g_{o} \in \mathbb{H}^{n}$. The horizontal plane $H_{g_{o}}$ passing through $g_{o}$ is the hyperplane in $\mathbb{H}^{n}$ spanned by $\left\{X_{1}\left(g_{o}\right), \ldots, X_{2 n}\left(g_{o}\right)\right\}$. One easily verifies that

$$
H_{g_{o}}=\left\{g=(x, y, t) \in \mathbb{H}^{n} \mid t=t_{o}+2<x, y_{o}>-2<x_{o}, y>\right\} .
$$

We denote by $H_{g_{o}}^{+}$the horizontal upper half-space at $g_{o}$ defined by

$$
H_{g_{o}}^{+}=\left\{g=(x, y, t) \in \mathbb{H}^{n} \mid t \geq t_{o}+2<x, y_{o}>-2<x_{o}, y>\right\}
$$

Returning to the example of the function $u(x, y, t)=t$ in $\mathbb{H}^{n}$, we see from (4.2) that $u$ satisfies the $H$-convexity inequality in Definition 3.2 provided that we confine the point $g^{\prime}$ to belong to the horizontal upper half-space at $g$. This leads us to the following definition.

Definition 4.6. A function $u: \mathbb{H}^{n} \rightarrow(-\infty, \infty]$ will be called mildly $H$ convex if it is proper, and if for every $g \in \mathbb{H}^{n}$ one has for every $0 \leq \lambda \leq 1$

$$
u\left(g_{\lambda}\right) \leq u(g)+\lambda\left(u\left(g^{\prime}\right)-u(g)\right)
$$

for every $g^{\prime} \in H_{g}^{+}$. The effective domain of $u$ is the set

$$
\operatorname{dom}_{H} u=\{g \in \boldsymbol{G} \mid u(g)<\infty\} .
$$

We denote by $\mathcal{C}_{H}^{m}\left(\mathbb{H}^{n}\right)$ the collection of all mildly $H$-convex functions on $\mathbb{H}^{n}$. 
It is clear from the above considerations that $u(x, y, t)=t$ is mildly $H$ convex, and, thanks to Proposition 3.5, so is the function $v(x, y, t)=\left(t_{+}\right)^{p}$, $p \geq 1$, where $t_{+}=\max (0, t)$. However, the function $u(g)=|t|^{p}, p \geq 1$ is not in $\mathcal{C}_{H}^{m}\left(\mathbb{H}^{n}\right)$. To see this, assume $p=1$, and consider in $\mathbb{H}^{1}$ the point $g=(x, y, 0)$. Taking $g^{\prime}=\left(x^{\prime}, y^{\prime}, 0\right)$ such that $x^{\prime} y-x y^{\prime}<0$, from Proposition 4.5 we see that $g^{\prime}$ belongs to the interior of $H_{g}^{+}$. Using the formula (4.1), a simple computation shows that for $u(g)=|t|$ the inequality

$$
u\left(g_{\lambda}\right) \leq u(g)+\lambda\left(u\left(g^{\prime}\right)-u(g)\right)
$$

now reads

$$
2 \lambda(1-\lambda)\left|x^{\prime} y-x y^{\prime}\right| \leq 0,
$$

which is clearly impossible, except in the trivial cases $\lambda=0,1$. We observe, however, that if we impose to $g^{\prime}=\left(x^{\prime}, y^{\prime}, 0\right)$ to be on the horizontal plane through $g=(x, y, 0)$, then $\left|x^{\prime} y-x y^{\prime}\right|=0$, and therefore the inequality is satisfied for all $\lambda \in[0,1]$. This negative example brings us to the subject of the next section.

\section{Weakly convex functions: The horizontal Hessian.}

Our starting point presently is the classical characterization of convexity in terms of the semi-definite positiveness of the Hessian matrix of a function. Such result suggests that a satisfactory notion of convexity in Carnot groups should be based on an analogous property of the intrinsic Hessian. The latter is defined as follows.

Definition 5.1. Let $\boldsymbol{G}$ be a Carnot group. Given $u: \boldsymbol{G} \rightarrow \mathbb{R}$, with $u \in$ $\Gamma^{2}(\boldsymbol{G})$, the horizontal Hessian of $u$ at $g \in \boldsymbol{G}$ is the $m \times m$ matrix $X^{2} u(g)=$ $\left(X_{i} X_{j} u(g)\right)_{i, j=1, \ldots, m}$. The symmetrized horizontal Hessian is defined by

$$
\left[X^{2} u(g)\right]^{\star}=\frac{1}{2}\left\{X^{2} u(g)+X^{2} u(g)^{T}\right\} .
$$

As we will shortly see, the analysis of the semi-definiteness of the matrix $\left[X^{2} u\right]^{\star}$ will lead us to yet another notion of convexity. To free the presentation of technical details, we consider at first the model situation of the Heisenberg group $\mathbb{H}^{1}$. Given $u \in C^{2}\left(\mathbb{H}^{1}\right)$, and two arbitrary, but fixed, points $g=(x, y, t), g^{\prime}=\left(x^{\prime}, y^{\prime}, t^{\prime}\right)$ in $\mathbb{H}^{1}$, we introduce the function $\phi:[0,1] \rightarrow \mathbb{R}$ defined by

$$
\phi(\lambda)=u\left(g_{\lambda}\right)
$$


where $g_{\lambda}$ is like in (4.1). Clearly, $\phi \in C^{2}(0,1)$ and moreover

$$
\phi(0)=u(g), \quad \phi(1)=u\left(g^{\prime}\right), \quad \phi^{\prime}(0)=<X u(g), x\left(g^{\prime}\right)-x(g)>.
$$

Keeping in mind (5.2), applying the standard Taylor formula to $\phi(\lambda)$ gives

$$
u\left(g^{\prime}\right)-u(g)-<X u(g), x\left(g^{\prime}\right)-x(g)>=\int_{0}^{1}(1-\lambda) \phi^{\prime \prime}(\lambda) d \lambda .
$$

We are interested in the explicit expression of $\phi^{\prime \prime}(\lambda)$. We have

$\phi^{\prime}(\lambda)=X_{1} u\left(g_{\lambda}\right)\left(x^{\prime}-x\right)+X_{2} u\left(g_{\lambda}\right)\left(y^{\prime}-y\right)+2 \lambda u_{t}\left(g_{\lambda}\right)\left\{t^{\prime}-t+2 x y^{\prime}-2 x^{\prime} y\right\}$.

A calculation based on (5.4) now gives

$$
\begin{aligned}
\phi^{\prime \prime}(\lambda) & =X_{1}^{2} u\left(g_{\lambda}\right)\left(x^{\prime}-x\right)^{2}+X_{1} X_{2} u\left(g_{\lambda}\right)\left(x^{\prime}-x\right)\left(y^{\prime}-y\right) \\
& +X_{2} X_{1} u\left(g_{\lambda}\right)\left(x^{\prime}-x\right)\left(y^{\prime}-y\right)+X_{2}^{2} u\left(g_{\lambda}\right)\left(y^{\prime}-y\right)^{2} \\
& +2\left(t^{\prime}-t+2\left(x y^{\prime}-x^{\prime} y\right)\right)\left\{u_{t}\left(g_{\lambda}\right)\right. \\
& +2 \lambda\left[X_{1}\left(u_{t}\right)\left(g_{\lambda}\right)\left(x^{\prime}-x\right)+X_{2}\left(u_{t}\right)\left(g_{\lambda}\right)\left(y^{\prime}-y\right)\right] \\
& \left.+2 \lambda^{2}\left(t^{\prime}-t+2\left(x y^{\prime}-x^{\prime} y\right)\right) u_{t t}\left(g_{\lambda}\right)\right\} .
\end{aligned}
$$

This formula reveals a remarkable property. If we confine the point $g^{\prime}$ to belong to the horizontal plane $H_{g}$ passing through $g$, then in view of Proposition 4.5 we have $t^{\prime}-t+2\left(x y^{\prime}-x^{\prime} y\right)=0$, and the equation (5.5) reduces to

$$
\begin{aligned}
\phi^{\prime \prime}(\lambda) & =X_{1}^{2} u\left(g_{\lambda}\right)\left(x^{\prime}-x\right)^{2}+X_{1} X_{2} u\left(g_{\lambda}\right)\left(x^{\prime}-x\right)\left(y^{\prime}-y\right) \\
& +X_{2} X_{1} u\left(g_{\lambda}\right)\left(x^{\prime}-x\right)\left(y^{\prime}-y\right)+X_{2}^{2} u\left(g_{\lambda}\right)\left(y^{\prime}-y\right)^{2} \\
& =<\left(X^{2} u\left(g_{\lambda}\right)\right)^{\star} \zeta, \zeta>
\end{aligned}
$$

where we have let $\zeta^{T}=\left(x^{\prime}-x, y^{\prime}-y\right)$. We have thus established the following result.

Proposition 5.2. Let $u \in \Gamma^{2}\left(\mathbb{H}^{n}\right)$. For any fixed $g=(x, y, t) \in \mathbb{H}^{n}$ and every $g^{\prime}=\left(x^{\prime}, y^{\prime}, t^{\prime}\right) \in H_{g}$ one has

$$
\phi^{\prime \prime}(\lambda)=<\left(X^{2} u\left(g_{\lambda}\right)\right)^{\star} \zeta, \zeta>
$$

where $\zeta^{T}=\left(x^{\prime}-x, y^{\prime}-y\right) \in \mathbb{R}^{2 n}$. 
Proof. Consider a family of compactly supported, smooth approximations to the identity $\left\{K_{\epsilon}\right\}_{\epsilon>0}$. For every $\epsilon>0$ the function $u_{\epsilon}=K_{\epsilon} \star u$ belongs to $C^{\infty}$. Moreover, thanks to the hypothesis $u \in \Gamma^{2}\left(\mathbb{H}^{n}\right)$ we have $u_{\epsilon} \rightarrow u$, $X_{j} u_{\epsilon} \rightarrow X_{j} u, X_{i} X_{j} u_{\epsilon} \rightarrow X_{i} X_{j} u$, uniformly on compact subsets of $\mathbb{H}^{n}$. Setting $\phi_{\epsilon}(\lambda)=u_{\epsilon}\left(g_{\lambda}\right)$, we obtain as in (5.6)

$$
\phi_{\epsilon}^{\prime \prime}(\lambda)=<\left(X^{2} u_{\epsilon}\left(g_{\lambda}\right)\right)^{\star} \zeta, \zeta>.
$$

Letting $\epsilon \rightarrow 0$ we reach the conclusion.

Proposition 5.2 continues to be valid for any Carnot group. We will need the following useful lemma from [GV1].

Lemma 5.3. Let $G$ be a Carnot group of step 2, then for every $\alpha, \alpha^{\prime}=$ $1, \ldots, m$, one has

$$
\begin{aligned}
X_{\alpha} & =\frac{\partial}{\partial x_{\alpha}}+\frac{1}{2} \sum_{\beta=1}^{k}<\left[\xi_{1}, X_{\alpha}\right], Y_{\beta}>\frac{\partial}{\partial y_{\beta}} \\
& =\frac{\partial}{\partial x_{\alpha}}+\frac{1}{2} \sum_{\beta=1}^{k} \sum_{\alpha^{\prime}=1}^{m} b_{\alpha^{\prime} \alpha}^{\beta} x_{\alpha^{\prime}}(g) \frac{\partial}{\partial y_{\beta}} . \\
X_{\alpha} X_{\alpha^{\prime}}= & \frac{\partial^{2}}{\partial x_{\alpha} \partial x_{\alpha^{\prime}}}+\frac{1}{2} \sum_{\beta=1}^{k}<\left[X_{\alpha}, X_{\alpha^{\prime}}\right], Y_{\beta}>\frac{\partial}{\partial y_{\beta}} \\
& +\frac{1}{2} \sum_{\beta=1}^{k}<\left[\xi_{1}, X_{\alpha^{\prime}}\right], Y_{\beta}>\frac{\partial^{2}}{\partial x_{\alpha} \partial y_{\beta}} \\
& +\frac{1}{2} \sum_{\beta=1}^{k}<\left[\xi_{1}, X_{\alpha}\right], Y_{\beta}>\frac{\partial^{2}}{\partial x_{\alpha^{\prime}} \partial y_{\beta}} \\
& +\frac{1}{4} \sum_{\beta, \beta^{\prime}=1}^{k}<\left[\xi_{1}, X_{\alpha}\right], Y_{\beta}><\left[\xi_{1}, X_{\alpha^{\prime}}\right], Y_{\beta^{\prime}}>\frac{\partial^{2}}{\partial y_{\beta} \partial y_{\beta^{\prime}}}
\end{aligned}
$$

From (5.9) we obtain the commutator formula

$$
\left[X_{\alpha}, X_{\alpha^{\prime}}\right] u=\sum_{\beta=1}^{k} b_{\alpha^{\prime} \alpha}^{\beta} \frac{\partial}{\partial y_{\beta}}
$$


Proof. Equation (5.8) is formula (3.3) in [GV1]. The formula (5.9) follows from (5.8), once we observe that

$$
\frac{\partial}{\partial y_{\beta}}<\left[\xi_{1}, X_{\alpha}\right], Y_{\beta^{\prime}}>=0
$$

and that

$$
\frac{\partial}{\partial x_{\alpha}}<\left[\xi_{1}, X_{\alpha^{\prime}}\right], Y_{\beta^{\prime}}>=\sum_{\gamma=1}^{m} \frac{\partial}{\partial x_{\alpha}} x_{\gamma}<\left[X_{\gamma}, X_{\alpha^{\prime}}\right], Y_{\beta^{\prime}}>=<\left[X_{\alpha}, X_{\alpha^{\prime}}\right], Y_{\beta^{\prime}}>\text {. }
$$

In the case of arbitrary step one has to replace formulas (5.8), (5.9) with more complicated ones. Let $m_{s}=\operatorname{dim} V_{s}, s=1, \ldots, r$, and indicate with $X_{1}, \ldots, X_{m_{1}}$ an orthonormal basis of $V_{1}$. We denote by $\left(x_{1}(g), \ldots, x_{r}(g)\right)$ the exponential coordinates of $g=\exp \left(\xi_{1}(g)+\ldots+\xi_{r}(g)\right)$, where for each $s=$ $1, \ldots, r$ we have presently let $x_{s}(g)=\left(x_{s, 1}(g), \ldots, x_{s, m_{s}}(g)\right)$ for the components of $\xi_{s}(g)$ with respect to a fixed orthonormal basis of $V_{s}$. The following formula holds

$$
X_{\alpha}=\frac{\partial}{\partial x_{1, \alpha}}+\sum_{s=2}^{r} \sum_{\beta=1}^{m_{s}} b_{s, \alpha}^{\beta}\left(x_{1}(g), \ldots, x_{s-1}(g)\right) \frac{\partial}{\partial x_{s, \beta}}, \quad \alpha=1, \ldots, m_{1},
$$

where each $b_{s, \alpha}^{\beta}$ is a homogeneous polynomial of weighted degree $s-1$. By weighted degree we mean that, as previously mentioned, the layer $V_{s}$, $s=1, \ldots, r$, in the stratification of $\mathfrak{g}$ is assigned the formal degree $s$. Correspondingly, each homogeneous monomial $x_{1}^{\alpha_{1}} x_{2}^{\alpha_{2}} \ldots x_{r}^{\alpha_{r}}$, with multi-indices $\alpha_{s}=\left(\alpha_{1, s}, \ldots, \alpha_{m_{s}, s}\right), s=1, \ldots, r$, is said to have weighted degree $p$ if

$$
\sum_{s=1}^{r} s\left(\sum_{\beta=1}^{m_{s}} \alpha_{\beta, s}\right)=p
$$

Formula (5.11) is proved using (2.7), analogously to (5.8), and we leave the details to the interested reader.

Proposition 5.4. Let $\boldsymbol{G}$ be a Carnot group and let $u \in \Gamma^{2}(\boldsymbol{G})$. For every fixed $g \in \boldsymbol{G}$, and for any $g^{\prime} \in H_{g}$ one has

$$
\phi^{\prime}(\lambda)=\sum_{\alpha=1}^{m} X_{\alpha} u\left(g_{\lambda}\right)\left(x_{\alpha}\left(g^{\prime}\right)-x_{\alpha}(g)\right)
$$




$$
\phi^{\prime \prime}(\lambda)=<\left(X^{2} u\left(g_{\lambda}\right)\right)^{\star} \zeta, \zeta>
$$

where $\zeta=\xi_{1}\left(g^{\prime}\right)-\xi_{1}(g) \in V_{1}$.

Proof. Again, it suffices to consider the case $u \in C^{2}(\boldsymbol{G})$. We give the details in the case of a group of step $r=2$. If $r \geq 3$ the proof proceeds along the same lines and we omit it. Suppose then that $g^{\prime} \in H_{g}$. Using the equations (4.7) and (4.10) we obtain for the function $\phi(\lambda)$ defined in (5.1)

$\phi(\lambda)=u\left(x_{\alpha}(g)+\lambda\left(x_{\alpha}\left(g^{\prime}\right)-x_{\alpha}(g)\right), y_{\beta}(g)+\frac{\lambda}{2} \sum_{\alpha, \alpha^{\prime}=1}^{m} b_{\alpha \alpha^{\prime}}^{\beta} x_{\alpha}(g) x_{\alpha^{\prime}}\left(g^{\prime}\right)\right)$,

where we have abused the notation by writing $x_{\alpha}(g)+\lambda\left(x_{\alpha}\left(g^{\prime}\right)-x_{\alpha}(g)\right)$ instead of $x_{1}(g)+\lambda\left(x_{1}\left(g^{\prime}\right)-x_{1}(g)\right), \ldots, x_{m}(g)+\lambda\left(x_{m}\left(g^{\prime}\right)-x_{m}(g)\right)$, and similarly for the variables in the second layer. Differentiating the latter equation with respect to $\lambda$ we find

$\phi^{\prime}(\lambda)=\sum_{\alpha=1}^{m} \frac{\partial u}{\partial x_{\alpha}}\left(g_{\lambda}\right)\left(x_{\alpha}\left(g^{\prime}\right)-x_{\alpha}(g)\right)+\frac{1}{2} \sum_{\beta=1}^{k} \sum_{\alpha, \alpha^{\prime}=1}^{m} b_{\alpha \alpha^{\prime}}^{\beta} x_{\alpha}(g) x_{\alpha^{\prime}}\left(g^{\prime}\right) \frac{\partial u}{\partial y_{\beta}}\left(g_{\lambda}\right)$

Using (5.8) we can rewrite the latter equation as follows

$$
\begin{aligned}
& \phi^{\prime}(\lambda)=\sum_{\alpha=1}^{m} X_{\alpha} u\left(g_{\lambda}\right)\left(x_{\alpha}\left(g^{\prime}\right)-x_{\alpha}(g)\right) \\
& +\frac{1}{2} \sum_{\beta=1}^{k} \sum_{\alpha, \alpha^{\prime}=1}^{m} b_{\alpha \alpha^{\prime}}^{\beta} x_{\alpha}(g) x_{\alpha^{\prime}}\left(g^{\prime}\right) \frac{\partial u}{\partial y_{\beta}}\left(g_{\lambda}\right) \\
& -\frac{1}{2} \sum_{\beta=1}^{k} \sum_{\alpha, \alpha^{\prime}=1}^{m} b_{\alpha^{\prime} \alpha}^{\beta}\left(x_{\alpha^{\prime}}(g)+\lambda\left(x_{\alpha^{\prime}}\left(g^{\prime}\right)-x_{\alpha^{\prime}}(g)\right)\right)\left(x_{\alpha}\left(g^{\prime}\right)-x_{\alpha}(g)\right) \frac{\partial u}{\partial y_{\beta}}\left(g_{\lambda}\right)
\end{aligned}
$$


Using (4.9) we see that

$$
\begin{aligned}
& -\frac{1}{2} \sum_{\beta=1}^{k} \sum_{\alpha, \alpha^{\prime}=1}^{m} b_{\alpha^{\prime} \alpha}^{\beta}\left(x_{\alpha^{\prime}}(g)+\lambda\left(x_{\alpha^{\prime}}\left(g^{\prime}\right)-x_{\alpha^{\prime}}(g)\right)\right)\left(x_{\alpha}\left(g^{\prime}\right)-x_{\alpha}(g)\right) \frac{\partial u}{\partial y_{\beta}}\left(g_{\lambda}\right) \\
& =-\frac{1}{2} \sum_{\beta=1}^{k} \sum_{\alpha, \alpha^{\prime}=1}^{m} b_{\alpha^{\prime} \alpha}^{\beta} x_{\alpha^{\prime}}(g) x_{\alpha}\left(g^{\prime}\right) \frac{\partial u}{\partial y_{\beta}}\left(g_{\lambda}\right) \\
& +\frac{\lambda}{2} \sum_{\beta=1}^{k} \sum_{\alpha, \alpha^{\prime}=1}^{m} b_{\alpha^{\prime} \alpha}^{\beta} x_{\alpha^{\prime}}\left(g^{\prime}\right) x_{\alpha}(g) \frac{\partial u}{\partial y_{\beta}}\left(g_{\lambda}\right) \\
& +\frac{\lambda}{2} \sum_{\beta=1}^{k} \sum_{\alpha, \alpha^{\prime}=1}^{m} b_{\alpha^{\prime} \alpha}^{\beta} x_{\alpha^{\prime}}(g) x_{\alpha}\left(g^{\prime}\right) \frac{\partial u}{\partial y_{\beta}}\left(g_{\lambda}\right) \\
& =-\frac{1}{2} \sum_{\beta=1}^{k} \sum_{\alpha, \alpha^{\prime}=1}^{m} b_{\alpha \alpha^{\prime}}^{\beta} x_{\alpha}(g) x_{\alpha^{\prime}}\left(g^{\prime}\right) \frac{\partial u}{\partial y_{\beta}}\left(g_{\lambda}\right),
\end{aligned}
$$

where we have used the equality $b_{\alpha \alpha^{\prime}}^{\beta}=-b_{\alpha^{\prime} \alpha}^{\beta}$. Substituting (5.15) in (5.14) we conclude that (5.12) holds.

From (5.12) we now easily obtain

$$
\phi^{\prime \prime}(\lambda)=\sum_{\alpha, \alpha^{\prime}=1}^{m} X_{\alpha} X_{\alpha^{\prime}} u\left(g_{\lambda}\right)\left(x_{\alpha}\left(g^{\prime}\right)-x_{\alpha}(g)\right)\left(x_{\alpha^{\prime}}\left(g^{\prime}\right)-x_{\alpha^{\prime}}(g)\right),
$$

which completes the proof.

Proposition (5.4) paves the way to the following definition, which plays a central role in this paper.

Definition 5.5. Let $\boldsymbol{G}$ be a Carnot group. A function $u: \boldsymbol{G} \rightarrow(-\infty, \infty]$ will be called weakly $H$-convex if it is proper, and if for any $g \in \boldsymbol{G}$ one has for every $0 \leq \lambda \leq 1$

$$
u\left(g_{\lambda}\right) \leq u(g)+\lambda\left(u\left(g^{\prime}\right)-u(g)\right),
$$

for every $g^{\prime} \in H_{g}$. When the strict inequality holds we say that $u$ is strictly weakly $H$-convex. The effective domain of $u$ is the set

$$
\operatorname{dom}_{H} u=\{g \in \boldsymbol{G} \mid u(g)<\infty\} .
$$

We denote by $\mathcal{C}_{H}^{w}(\boldsymbol{G})$ the class of all weakly $H$-convex functions on $\boldsymbol{G}$. 
Remark 5.6. It is worth noting at this point that Definition 5.5 involves a one-sided information on $u$ on lower-dimensional manifolds, namely the horizontal planes. This new aspect, which is of course not present in the classical theory of convex functions, is a source of difficulties when one wants to pass from such information to conclusions on a set of full measure.

When $u$ is sufficiently smooth we obtain the following basic consequence of Definition 5.5.

Proposition 5.7. Suppose that $u: G \rightarrow \mathbb{R}$ is weakly $H$-convex. If $u \in$ $\Gamma^{1}(\boldsymbol{G})$ one has for any fixed $g \in \boldsymbol{G}$

$$
<X u(g), \xi_{1}\left(g^{\prime}\right)-\xi_{1}(g)>+u(g) \leq u\left(g^{\prime}\right) \quad \text { for every } \quad g^{\prime} \in H_{g} .
$$

Proof. From Definition 5.5 we have

$$
\frac{u\left(g_{\lambda}\right)-u(g)}{\lambda} \leq u\left(g^{\prime}\right)-u(g)
$$

We now pass to the limit for $\lambda \rightarrow 0$ obtaining the conclusion from Proposition 2.3.

Geometric interpretation of weak $H$-convexity. Proposition 5.7 suggests the following geometric interpretation of the notion of weak $\mathrm{H}$ convexity which avoids reference to the sub-Riemannian gradient of $u$, and only involves the subbundle of the horizontal planes. Let us assume that $u \in C^{1}(\boldsymbol{G})$, and for every $g$ introduce a new function $U_{g}: H_{g} \rightarrow \mathbb{R}$, defined by restricting $u$ to $H_{g}$. Using exponential coordinates we can think of $U_{g}$ as defined on the embedded submanifold of the Lie algebra $\mathfrak{g}$ corresponding to $H_{g}$. With this identification $U_{g}$ becomes a function of the coordinates $\xi_{1}\left(g^{\prime}\right)$, projection of the exponential coordinates $\xi\left(g^{\prime}\right)$ onto the first layer $V_{1}$. Following the arguments above one easily recognizes that

$$
X u(g)=D U_{g}\left(\xi_{1}(g)\right),
$$

where we have denoted by $D U_{g}$ the standard gradient of $U_{g}$ with respect to the variables $\xi_{1}$ (we stress that (5.16) only holds at the base point $g$, it does not hold at other points $g^{\prime} \in H_{g}$ ). Therefore, the conclusion of Proposition 5.7 can be reformulated in the following way

$$
<D U_{g}\left(\xi_{1}\right), \xi_{1}^{\prime}-\xi_{1}>+U_{g}\left(\xi_{1}\right) \leq U_{g}\left(\xi_{1}^{\prime}\right) \quad \text { for every } \quad \xi_{1}^{\prime} \in V_{1}
$$


In other words, a function $u \in C^{1}(\boldsymbol{G})$ is weakly $H$-convex if and only if for every $g \in \boldsymbol{G}$ the graph of the restriction of $u$ to $H_{g}$ lies above its tangent plane at $\xi_{1}(g)$.

The notion of weak $H$-convexity has been conceived to include the function $u(g)=t$ in the Heisenberg group $\mathbb{H}^{n}$. In this perspective, the next useful proposition should come as no surprise.

Proposition 5.8. Let $\psi: \mathbb{R} \rightarrow \mathbb{R}$ be convex, then the function $u: \mathbb{H}^{n} \rightarrow \mathbb{R}$ defined by $u(g)=\psi(t)$ belongs to $\mathcal{C}_{H}^{w}(\boldsymbol{G})$. In particular, $u(g)=|t|^{p}, p \geq 1$ is in $\mathcal{C}_{H}^{w}(\boldsymbol{G})$.

Proof. It is an immediate application of Definition 5.5, once we observe that, thanks to Proposition 4.4 , for any $g \in \mathbb{H}^{n}$ one has $g \delta_{\lambda}\left(g^{-1} g^{\prime}\right)=$ $(1-\lambda) g+\lambda g^{\prime}$ if $g^{\prime} \in H_{g}$. Here, the right-hand side of the latter equation represents the Euclidean convex combination of $g$ and $g^{\prime}$, as points of $\mathbb{R}^{2 n+1}$.

Before proceeding, it is worth noting the following immediate consequence of the definitions and of the examples $u(g)=t$, and $u(g)=|t|$, see Section 4 and Proposition 5.8:

$$
\mathcal{C}_{H}^{s} \quad \subsetneq \quad \mathcal{C}_{H}^{m} \quad \subsetneq \quad \mathcal{C}_{H}^{w}
$$

Remark 5.9. It is important to notice that Propositions 3.4, 3.5 and 3.9 continue to be valid if in their statements we replace "strongly $H$-convex" with "weakly $H$-convex".

Proposition 5.10. A function $u: G \rightarrow(-\infty, \infty]$ is weakly $H$-convex if and only if such is $L_{h} u(g)=u(h g)$, for every $h \in \boldsymbol{G}$.

Proof. We argue similarly to the proof of Proposition 3.3, noting in addition that for any $g^{\prime} \in H_{g}$, one has $h g^{\prime} \in H_{h g}$.

The following two theorems provide the main motivation for Definition 5.5 : the weak $H$-convexity of a function in the class $\Gamma^{2}$ is characterized by the positivity of its horizontal Hessian. To facilitate the presentation we will first analyze with full details the Heisenberg group model in Theorem 5.11. 
Theorem 5.11. A function $u \in \Gamma^{2}\left(\mathbb{H}^{n}\right)$ is weakly $H$-convex if and only if the symmetrized horizontal Hessian $\left(X^{2} u(g)\right)^{\star}$ is semi-definite positive at every $g \in \mathbb{H}^{n}$.

Proof. We first prove the necessity. Suppose that $u \in \Gamma^{2}\left(\mathbb{H}^{n}\right)$ be weakly $H$-convex. If we fix $g \in \mathbb{H}^{n}$, from Proposition 5.7 we obtain

$$
u\left(g^{\prime}\right) \geq u(g)+<X u(g), \xi_{1}\left(g^{\prime}\right)-\xi_{1}(g)>, \quad \text { for every } \quad g^{\prime} \in H_{g} .
$$

From (5.19), (5.3) and Proposition 5.2 we conclude

$$
0 \leq \int_{0}^{1}(1-\lambda)<\left(X^{2} u\left(g_{\lambda}\right)\right)^{\star} \zeta, \zeta>d \lambda
$$

where $\zeta^{T}=\left(x^{\prime}-x, y^{\prime}-y\right) \in \mathbb{R}^{2 n}$.

At this point, given any vector $\zeta=(\xi, \eta)^{T} \in \mathbb{R}^{2 n}$, and any $0<\tau<1$, we choose $g^{\prime}=\left(x^{\prime}, y^{\prime}, t^{\prime}\right) \in H_{g}$ such that $g^{-1} g^{\prime}=\delta_{\tau}(\xi, \eta, 0) \stackrel{\text { def }}{=} \delta_{\tau} \tilde{\zeta}$. In other words, we want to take

$x^{\prime}-x=\tau \xi, \quad y^{\prime}-y=\tau \eta, \quad t^{\prime}-t+2\left(<x, y^{\prime}>-<x^{\prime}, y>\right)=0$.

This can of course be accomplished by choosing

$x^{\prime}=x+\tau \xi, \quad y^{\prime}=y+\tau \eta, \quad t^{\prime}=t+2 \tau(<\xi, y>-<x, \eta>)$.

Inserting this choice in (5.20), we find

$$
0 \leq \int_{0}^{1}(1-\lambda)<\left(X^{2} u\left(g \delta_{\lambda}\left(\delta_{\tau} \tilde{\zeta}\right)\right)\right)^{\star} \zeta, \zeta>d \lambda
$$

Noting that $g \delta_{\lambda}\left(\delta_{\tau} \tilde{\zeta}\right) \rightarrow g$, as $\tau \rightarrow 0$, and using the continuity of the second horizontal derivatives of $u$, we conclude from (5.21)

$$
0 \leq<\left(X^{2} u(g)\right)^{\star} \zeta, \zeta>
$$

which establishes the first part of the theorem.

Suppose now that (5.22) holds for any $g \in \mathbb{H}^{n}$ and for every $\zeta=(\xi, \eta)^{T} \in$ $\mathbb{R}^{2 n}$. We will prove that $u$ is weakly $H$-convex. We fix $g \in \mathbb{H}^{n}$ and consider an arbitrary $g^{\prime} \in H_{g}$. Proposition 5.2 implies

$$
\phi^{\prime \prime}(\lambda)=<\left(X^{2} u\left(g_{\lambda}\right)\right)^{\star} \zeta, \zeta>
$$


where now $\zeta^{T}=\left(x^{\prime}-x, y^{\prime}-y\right)$. According to the hypothesis we have $\phi^{\prime \prime}(\lambda) \geq 0$. Since $\phi \in C^{2}(0,1)$, we infer that $\phi:[0,1] \rightarrow \mathbb{R}$ is convex. This gives in particular

$$
\phi(\lambda) \leq(1-\lambda) \phi(0)+\lambda \phi(1), \quad \text { for every } \quad 0 \leq \lambda \leq 1 .
$$

In terms of $u$ the latter inequality can be written as

$$
u\left(g_{\lambda}\right) \leq(1-\lambda) u(g)+\lambda u\left(g^{\prime}\right)
$$

which proves that $u$ is weakly $H$-convex.

Theorem 5.11 continues to be valid in any Carnot group $\boldsymbol{G}$. The proof is analogous to that of Theorem 5.11 and we leave the details to the interested reader. We only mention that in the proof of the necessity, similarly to the case of $\mathbb{H}^{n}$ one is confronted with the task of choosing, for any given $\zeta \in V_{1}$, and any $\tau \in(0,1)$, a point $g^{\prime} \in \boldsymbol{G}$ such that $g^{-1} g^{\prime}=\delta_{\tau} \exp (\zeta)$, and for which $g^{\prime} \in H_{g}$. This can be accomplished similarly to before. For instance, if $\boldsymbol{G}$ is of step 2 , then if $\zeta=\sum_{\alpha=1}^{m} \zeta_{\alpha} X_{\alpha}$ we choose $g^{\prime} \cong\left(x\left(g^{\prime}\right), y\left(g^{\prime}\right)\right)$ as follows

$$
\begin{gathered}
x_{\alpha}\left(g^{\prime}\right)=x_{\alpha}(g)+\tau \zeta_{\alpha}, \quad \alpha=1, \ldots, m, \\
y_{\beta}\left(g^{\prime}\right)=y_{\beta}(g)+\frac{\tau}{2} \sum_{\alpha, \alpha^{\prime}=1}^{m} b_{\alpha \alpha^{\prime}}^{\beta} x_{\alpha}(g) \zeta_{\alpha^{\prime}} \quad \beta=1, \ldots, k .
\end{gathered}
$$

Theorem 5.12. In a Carnot group $\boldsymbol{G}$ a function $u \in \Gamma^{2}(\boldsymbol{G})$ is weakly $H$ convex if and only if the symmetrized horizontal Hessian $\left(X^{2} u(g)\right)^{\star}$ is semidefinite positive at every $g \in \boldsymbol{G}$.

We have seen in Proposition 5.8 that the function $u(g)=t^{2}$ in $\mathbb{H}^{n}$ is weakly $H$-convex. An interesting alternative proof of this result can be given applying Theorem 5.11. Confining our attention to $\mathbb{H}^{1}$, a simple computation gives

$$
\left(X^{2} u(g)\right)^{\star}=\left(\begin{array}{cc}
8 y^{2} & -8 x y \\
-8 x y & 8 x^{2}
\end{array}\right) .
$$

This implies for every $\zeta=(\xi, \eta)^{T}$

$$
<\left(X^{2} u(g)\right)^{\star} \zeta, \zeta>=8(x \eta-y \xi)^{2} \geq 0,
$$

which, according to Theorem 5.11 , proves that $u \in \mathcal{C}_{H}^{w}\left(\mathbb{H}^{1}\right)$. We also notice that

$$
\operatorname{det}\left(X^{2} u\right)^{\star} \equiv 0
$$


The geometric meaning of this fact is understood by looking at the restriction of the function $u(g)=t^{2}$ to the horizontal plane $H_{g_{o}}$, where $g_{o}=\left(x_{o}, y_{o}, t_{o}\right)$ is arbitrarily fixed in $\mathbb{H}^{1}$. Such restriction is given by

$$
U_{g_{o}}(x, y)=U(x, y)=\left(t_{o}+2 y_{o} x-2 x_{o} y\right)^{2} .
$$

The principal curvatures at $\left(x_{o}, y_{o}\right)$ of the graph of $U_{g_{o}}$ are given by the equation

$$
\kappa=H \pm \sqrt{H^{2}-K},
$$

where $H$ and $K$ respectively denote the mean curvature and the Gaussian curvature of the graph at $\left(x_{o}, y_{o}\right)$. Since

$$
K=\frac{U_{x x} U_{y y}-U_{x y}^{2}}{\left(1+U_{x}^{2}+U_{y}^{2}\right)^{2}}, \quad 2 H=\operatorname{div}\left(\frac{D U}{\sqrt{1+|D U|^{2}}}\right)
$$

we easily recognize that $K\left(x_{o}, y_{o}\right)=0, H\left(x_{o}, y_{o}\right)=4\left(x_{o}^{2}+y_{o}^{2}\right)$, and therefore

$$
\kappa_{1}\left(x_{o}, y_{o}\right)=8\left(x_{o}^{2}+y_{o}^{2}\right), \quad \kappa_{2}\left(x_{o}, y_{o}\right) \equiv 0 .
$$

We stress that when $g_{o}=\left(0,0, t_{o}\right)$ then the plane $H_{g_{o}}$ has a characteristic point at $g_{o}$ (i.e., a point where the vector fields $X_{1}$ and $X_{2}$ become tangent to the plane), and we have $\kappa_{1}=\kappa_{2}=0$. We will come back to the equation $\operatorname{det}\left(X^{2} u\right)^{\star}=F$ in Section 10 .

\section{Weak convexity of the gauge in groups of Heisenberg type.}

A remarkable class of Carnot groups is that of Heisenberg type introduced by Kaplan $[\mathrm{K}]$ in connection with questions of hypoellipticity. These include the Heisenberg group and arise as the nilpotent component $N$ in the Iwasawa decomposition $K A N$ of simple groups of rank one [CDKR]. Due to their intrinsic symmetries, the natural non-isotropic gauge (6.3) plays a predominant role in study of the various linear and nonlinear differential operators, see (6.4), (6.6), Proposition 6.4 and Theorem 10.9. On the other hand, such function also serves as a true distance [Cy] (not just a pseudodistance), equivalent to the Carnot-Carathéodory metric associated with the basis $\left\{X_{1}, \ldots, X_{m}\right\}$. It is thus natural to study its convexity properties in view to applications to problems in optimal control and fully nonlinear equations.

In this section we establish a basic property of groups of Heisenberg type, namely that their non-isotropic gauge is a weakly $H$-convex function. This is the counterpart of the equally basic, yet trivial fact, that the distance from 
the origin in a normed linear space is an ordinary convex function. By contrast, the weak $H$-convexity of the gauge is a highly nontrivial phenomenon which has both geometric and analytic implications. Its proof is crucially based on Theorem 5.12, but one also needs to develop additional arguments to take care of the singularity of the gauge. We begin by introducing the relevant geometric ambients.

Definition 6.1. A Carnot group $\boldsymbol{G}$ of step 2 is called of Heisenberg type if for every $\eta \in V_{2}$, such that $|\eta|=1$, the map $J(\eta): V_{1} \rightarrow V_{1}$ is orthogonal.

In Definition 6.1 for a Carnot group $\boldsymbol{G}$ of step 2, with Lie algebra $\mathfrak{g}=$ $V_{1} \oplus V_{2}$, the mapping $J: V_{2} \rightarrow \operatorname{End}\left(V_{1}\right)$ is that defined in (4.4).

Definition 6.1 implies

$$
\begin{gathered}
|J(\eta) \xi|=|\eta||\xi|, \quad \eta \in V_{2}, \quad \xi \in V_{1}, \\
<J\left(\eta^{\prime}\right) \xi, J\left(\eta^{\prime \prime}\right) \xi>=<\eta^{\prime}, \eta^{\prime \prime}>|\xi|^{2}, \quad \eta^{\prime}, \eta^{\prime \prime} \in V_{2}, \quad \xi \in V_{1} .
\end{gathered}
$$

A basic property of groups of Heisenberg type is that the fundamental solution of a fixed sub-Laplacian is expressed by the appropriate power of the renormalized gauge

$$
N(g)=\left(|x(g)|^{4}+16|y(g)|^{2}\right)^{1 / 4} .
$$

Given the sub-Laplacian $\mathcal{L}=\sum_{j=1}^{m} X_{j}^{2}$ associated with an orthonormal basis of $V_{1}$, Kaplan $[\mathrm{K}]$ proved that there exists a constant $C=C(\boldsymbol{G})>0$ such that the locally integrable function

$$
\Gamma\left(g, g^{\prime}\right)=\frac{C}{N\left(g^{-1} g^{\prime}\right)^{Q-2}},
$$

satisfies the equation $\mathcal{L} \Gamma(g, \cdot)=-\delta_{g}$ in $\mathcal{D}^{\prime}(\boldsymbol{G})$. This result generalized Folland's fundamental solution for the Heisenberg group $\mathbb{H}^{n}[\mathrm{~F} 1]$. In [CDG] it was discovered that, remarkably, the gauge (6.3) enters also in the expression of the fundamental solution of the following one-parameter family of non-linear operators

$$
\mathcal{L}_{p} u=\sum_{j=1}^{m} X_{j}\left(|X u|^{p-2} X_{j} u\right), \quad 1<p<\infty .
$$


It was proved there that for every $1<p<\infty, p \neq Q$, there exists $C_{p}=C(\boldsymbol{G}, p)>0$ such that the fundamental solution of $-\mathcal{L}_{p}$ is given by

$$
\Gamma_{p}\left(g, g^{\prime}\right)=\frac{C_{p}}{N\left(g^{-1} g^{\prime}\right)^{(Q-p) /(p-1)}} .
$$

In the conformally invariant case $p=Q$ one has instead $\Gamma_{Q}\left(g, g^{\prime}\right)=$ $C \log N\left(g, g^{\prime}\right)$. This latter result was also independently found in [HH].

Similarly to its Euclidean counterpart, the one-parameter family of quasilinear operators $\mathcal{L}_{p}$ tends in a suitable sense, in the limit as $p \rightarrow \infty$, to the following nonlinear operator

$$
\mathcal{L}_{\infty} u \stackrel{\text { def }}{=} \sum_{\alpha, \alpha^{\prime}=1}^{m} X_{\alpha} X_{\alpha^{\prime}} u X_{\alpha} u X_{\alpha^{\prime}} u=<\left(X^{2} u\right)^{\star} X u, X u>
$$

For the Heisenberg group $\mathbb{H}^{n}$ a notion of viscosity solution of (6.7) has been recently introduced by $\mathrm{T}$. Bieske in his interesting paper [Bi]. The same author has also established the uniqueness of such solutions. It is remarkable that, once again, the gauge $N(g)$ defined by (6.3) constitutes a singular solution of (6.7), see Proposition 6.4. This result, which we discovered back in 1995, provides one further witness of the relevance of such function, and also has applications in various questions connected with unique continuation [GV2], and with trace inequalities [DGN1]. We recall the following useful formulas, see [CDG], [GV1].

Lemma 6.2. In a Carnot group $\boldsymbol{G}$ of step 2 , consider the function $\psi(g) \stackrel{\text { def }}{=}$ $|x(g)|^{2}$. For any $s=1, \ldots, k$, one has for every $g \in \boldsymbol{G}$

$$
<X \psi(g), X y_{s}(g)>\equiv 0 \text {. }
$$

As a consequence,

$$
<X \psi(g), X\left(|y|^{2}\right)(g)>=0 \text {, }
$$

and

$$
<\xi_{1}(g), X\left(|y|^{2}\right)(g)>=0 .
$$

The next lemma expresses some key symmetry properties of groups of Heisenberg type. 
Lemma 6.3. Let $\boldsymbol{G}$ be a group of Heisenberg type. One has

$$
\begin{gathered}
|X N(g)|^{2}=\frac{|x(g)|^{2}}{N(g)^{2}} . \\
\left|X\left(y_{l}\right)(g)\right|^{2}=\frac{1}{4}|x(g)|^{2}, \quad l=1, \ldots, k . \\
\left|X\left(|y|^{2}\right)(g)\right|^{2}=|x(g)|^{2}|y(g)|^{2} .
\end{gathered}
$$

Proposition 6.4. Let $\boldsymbol{G}$ be a group of Heisenberg type, then one has in the classical sense

$$
\mathcal{L}_{\infty} N=0, \quad \text { in } \quad \boldsymbol{G} \backslash\{e\} .
$$

Proof. We observe the following alternative form of (6.7)

$$
\mathcal{L}_{\infty} u=\frac{1}{2}<X\left(|X u|^{2}\right), X u>.
$$

Using (6.14) we find

$$
\mathcal{L}_{\infty} N=\frac{1}{2}<X\left(|X N|^{2}\right), X N>=\frac{1}{2}<X\left(N^{-2}|x|^{2}\right), X N>
$$

where in the latter equation we have used (6.11) in Lemma 6.3. We now have, again by (6.11),

$$
\begin{aligned}
<X\left(N^{-2}|x|^{2}\right), X N> & =-2 N^{-3}|x|^{2}<X N, X N> \\
& +2 N^{-2}<\xi_{1}, X N> \\
& =-2 N^{-5}|x|^{4}+2 N^{-2}<\xi_{1}, X N>
\end{aligned}
$$

Finally, we obtain

$$
X N=\frac{1}{4} N^{-3} X\left(\psi^{2}+16|y|^{2}\right)=N^{-3}\left\{|x|^{2} \xi_{1}+4 X|y|^{2}\right\},
$$

which gives

$$
<\xi_{1}, X N>=N^{-3}\left\{|x|^{4}+4<\xi_{1}, X|y|^{2}>\right\}=N^{-3}|x|^{4},
$$

where in the last equality we have used (6.10) in Lemma 6.2. Replacing the latter equation in (6.15) we reach the conclusion. 
Consider the function $u(g)=N(g)^{4}=|x(g)|^{4}+16|y(g)|^{2}$. From the results in Section 3, we easily conclude that $g \rightarrow|x(g)|^{4}$ is strongly $H$-convex, and therefore it is weakly $H$-convex, see (5.18). Next, we prove that also the function $g \rightarrow|y(g)|^{2}$ is weakly-convex, and therefore the fourth power of the gauge is weakly $H$-convex.

Proposition 6.5. In a Carnot group of step 2 the function $\chi(g) \stackrel{\text { def }}{=}|y(g)|^{2}$ belongs to $\mathcal{C}_{H}^{w}(\boldsymbol{G})$, therefore so does $u(g)=N(g)^{4}=|x(g)|^{4}+16|y(g)|^{2}$.

Proof. According to Lemma 5.3 we have

$$
\begin{aligned}
X_{\alpha} X_{\alpha^{\prime}} \chi & =\sum_{\beta=1}^{k}<\left[X_{\alpha}, X_{\alpha^{\prime}}\right], Y_{\beta}>y_{\beta} \\
& +\frac{1}{2} \sum_{\beta, \beta^{\prime}=1}^{k}<\left[\xi_{1}, X_{\alpha}\right], Y_{\beta}><\left[\xi_{1}, X_{\alpha^{\prime}}\right], Y_{\beta^{\prime}}>\delta_{\beta \beta^{\prime}} \\
& =\sum_{\beta=1}^{k}<\left[X_{\alpha}, X_{\alpha^{\prime}}\right], Y_{\beta}>y_{\beta} \\
& +\frac{1}{2} \sum_{\beta=1}^{k}<\left[\xi_{1}, X_{\alpha}\right], Y_{\beta}><\left[\xi_{1}, X_{\alpha^{\prime}}\right], Y_{\beta}>
\end{aligned}
$$

Since $\left[X_{\alpha}, X_{\alpha^{\prime}}\right]=-\left[X_{\alpha^{\prime}}, X_{\alpha}\right]$, we obtain

$$
\frac{1}{2}\left(X_{\alpha} X_{\alpha^{\prime}} \chi+X_{\alpha^{\prime}} X_{\alpha} \chi\right)=\frac{1}{2} \sum_{\beta=1}^{k}<\left[\xi_{1}, X_{\alpha}\right], Y_{\beta}><\left[\xi_{1}, X_{\alpha^{\prime}}\right], Y_{\beta}>.
$$

This gives for every $\zeta \in V_{1}$

$$
\begin{aligned}
& <\left(X^{2} \chi(g)\right)^{\star} \zeta, \zeta> \\
& =\frac{1}{2} \sum_{\beta=1}^{k} \sum_{\alpha, \alpha^{\prime}=1}^{m}<J\left(Y_{\beta}\right) \xi_{1}(g), X_{\alpha}>\zeta_{\alpha}<J\left(Y_{\beta}\right) \xi_{1}(g), X_{\alpha^{\prime}}>\zeta_{\alpha^{\prime}} \\
& =\frac{1}{2} \sum_{\beta=1}^{k}<J\left(Y_{\beta}\right) \xi_{1}(g), \zeta>^{2} \geq 0 .
\end{aligned}
$$

In view of Theorem 5.11 we conclude that $\chi \in \mathcal{C}_{H}^{w}$. 
Having established the weak $H$-convexity of $N^{4}$, the question remains open of whether the gauge itself is weakly $H$-convex. This is a delicate problem. The main step toward it consists in establishing the following important positivity result.

Theorem 6.6. Let $N(g)=\left(|x(g)|^{4}+16|y(g)|^{2}\right)^{1 / 4}$ be the gauge in a group of Heisenberg type, then for any given $g \in \boldsymbol{G} \backslash\{e\}$ one has

$$
<\left(X^{2} N(g)\right)^{\star} \zeta, \zeta>\geq 0
$$

for every $\zeta \in V_{1}$.

Proof. Let $\chi(g)=|y(g)|^{2}$, and consider the function $\psi(g)=|x(g)|^{2}$. We thus have $u(g)=N(g)^{4}=\psi(g)^{2}+16 \chi(g)$. We first compute the quadratic form associated with the symmetrized horizontal Hessian of $u$. Since

$$
\left(X^{2} u(g)\right)^{\star}=\left(X^{2} \psi^{2}(g)\right)^{\star}+16\left(X^{2} \chi(g)\right)^{\star},
$$

we need to compute $\left\langle\left(X^{2} \psi^{2}(g)\right)^{\star} \zeta, \zeta>\right.$ and $\left\langle\left(X^{2} \chi(g)\right)^{\star} \zeta, \zeta>\right.$, where $\zeta \in V_{1}$. The latter of these quantities has been obtained in (6.17), so we are left with computing the former. From (5.8), (5.9) in Lemma 5.3 we find

$$
\begin{gathered}
X_{\alpha^{\prime}} \psi^{2}=2 \psi X_{\alpha^{\prime}} \psi=4 x_{\alpha^{\prime}} \psi, \\
X_{\alpha} X_{\alpha^{\prime}} \psi^{2}=4 \delta_{\alpha \alpha^{\prime}} \psi+8 x_{\alpha} x_{\alpha^{\prime}} .
\end{gathered}
$$

From (6.21) and (6.17) we conclude

$$
<\left(X^{2} u\right)^{\star} \zeta, \zeta>=4\left|\xi_{1}\right|^{2}|\zeta|^{2}+8<\xi_{1}, \zeta>^{2}+8 \sum_{\beta=1}^{k}<J\left(Y_{\beta}\right) \xi_{1}, \zeta>^{2}
$$

Since $N=u^{1 / 4}$, an elementary computation gives

$$
X_{\alpha} X_{\alpha^{\prime}} N=\frac{1}{4 N^{3}}\left\{X_{\alpha} X_{\alpha^{\prime}} u-\frac{3}{4} \frac{X_{\alpha} u X_{\alpha^{\prime}} u}{N^{4}}\right\} \text {. }
$$

Keeping in mind (6.20) one easily recognizes that

$$
X_{\alpha} u X_{\alpha^{\prime}} u=16\left(\psi x_{\alpha}+4 X_{\alpha} \chi\right)\left(\psi x_{\alpha^{\prime}}+4 X_{\alpha^{\prime}} \chi\right) .
$$


On the other hand one obtains from (5.8)

$$
X_{\alpha} \chi=\sum_{\beta=1}^{k} y_{\beta}<\left[\xi_{1}, X_{\alpha}\right], Y_{\beta}>
$$

$$
=\sum_{\beta=1}^{k}<\xi_{2}, Y_{\beta}><\left[\xi_{1}, X_{\alpha}\right], Y_{\beta}>=<\left[\xi_{1}, X_{\alpha}\right], \xi_{2}>=<J\left(\xi_{2}\right) \xi_{1}, X_{\alpha}>.
$$

Substitution in the previous formula gives

$$
\begin{aligned}
X_{\alpha} u X_{\alpha^{\prime}} u & =16\left(\psi x_{\alpha}+4<J\left(\xi_{2}\right) \xi_{1}, X_{\alpha}>\right)\left(\psi x_{\alpha^{\prime}}+4<J\left(\xi_{2}\right) \xi_{1}, X_{\alpha^{\prime}}>\right) \\
& =16\left\{\left|\xi_{1}\right|^{4} x_{\alpha} x_{\alpha^{\prime}}+4\left|\xi_{1}\right|^{2} x_{\alpha}<J\left(\xi_{2}\right) \xi_{1}, X_{\alpha^{\prime}}>\right. \\
& +4\left|\xi_{1}\right|^{2} x_{\alpha^{\prime}}<J\left(\xi_{2}\right) \xi_{1}, X_{\alpha}> \\
& \left.+16<J\left(\xi_{2}\right) \xi_{1}, X_{\alpha}><J\left(\xi_{2}\right) \xi_{1}, X_{\alpha^{\prime}}>\right\} .
\end{aligned}
$$

From this expression, from (6.23) and from (6.22) we find

$$
\begin{aligned}
& <\left(X^{2} N\right)^{\star} \zeta, \zeta>=\frac{1}{4 N^{3}}\left\{<\left(X^{2} u\right)^{\star} \zeta, \zeta>-\frac{3}{4} \frac{\sum_{\alpha, \alpha^{\prime}=1}^{m} X_{\alpha} u X_{\alpha^{\prime}} u \zeta_{\alpha} \zeta_{\alpha^{\prime}}}{N^{4}}\right\} . \\
& =\frac{1}{N^{3}}\left\{\left|\xi_{1}\right|^{2}|\zeta|^{2}+2<\xi_{1}, \zeta>^{2}+2 \sum_{\beta=1}^{k}<J\left(Y_{\beta}\right) \xi_{1}, \zeta>^{2}\right. \\
& -\frac{3}{N^{4}}\left[\left|\xi_{1}\right|^{4}<\xi_{1}, \zeta>^{2}+8\left|\xi_{1}\right|^{2}<\xi_{1}, \zeta><J\left(\xi_{2}\right) \xi_{1}, \zeta>\right. \\
& \left.\left.+16<J\left(\xi_{2}\right) \xi_{1}, \zeta>^{2}\right]\right\} \\
& =\frac{1}{N^{3}}\left\{\left|\xi_{1}\right|^{2}|\zeta|^{2}+2<\xi_{1}, \zeta>^{2}+2 \sum_{\beta=1}^{k}<J\left(Y_{\beta}\right) \xi_{1}, \zeta>^{2}\right. \\
& \left.-\frac{3}{N^{4}}\left[\left|\xi_{1}\right|^{2}<\xi_{1}, \zeta>+4<J\left(\xi_{2}\right) \xi_{1}, \zeta>\right]^{2}\right\} .
\end{aligned}
$$

From (6.26) we see that proving the theorem is equivalent to showing 
that

$$
\begin{aligned}
& \frac{3}{N^{4}}\left[\left|\xi_{1}\right|^{2}<\xi_{1}, \zeta>+4<J\left(\xi_{2}\right) \xi_{1}, \zeta>\right]^{2} \\
& \leq\left|\xi_{1}\right|^{2}|\zeta|^{2}+2<\xi_{1}, \zeta>^{2}+2 \sum_{\beta=1}^{k}<J\left(Y_{\beta}\right) \xi_{1}, \zeta>^{2}
\end{aligned}
$$

Recalling that $\xi_{2}=\sum_{\beta=1}^{k} y_{\beta} Y_{\beta}$, we now have from Cauchy-Schwarz inequality

$$
\begin{aligned}
& {\left[\left|\xi_{1}\right|^{2}<\xi_{1}, \zeta>+4<J\left(\xi_{2}\right) \xi_{1}, \zeta>\right]^{2}} \\
& =\left[\left|\xi_{1}\right|^{2}<\xi_{1}, \zeta>+4 \sum_{\beta=1}^{k} y_{\beta}<J\left(Y_{\beta}\right) \xi_{1}, \zeta>\right]^{2} \\
& \leq\left(\left|\xi_{1}\right|^{4}+16\left|\xi_{2}\right|^{2}\right)\left(<\xi_{1}, \zeta>^{2}+\sum_{\beta=1}^{k}<J\left(Y_{\beta}\right) \xi_{1}, \zeta>^{2}\right) \\
& =N^{4}\left(<\xi_{1}, \zeta>^{2}+\sum_{\beta=1}^{k}<J\left(Y_{\beta}\right) \xi_{1}, \zeta>^{2}\right)
\end{aligned}
$$

Using (6.28) we see that in order to establish (6.27) it suffices to prove

$$
\begin{aligned}
& 3\left(<\xi_{1}, \zeta>^{2}+\sum_{\beta=1}^{k}<J\left(Y_{\beta}\right) \xi_{1}, \zeta>^{2}\right) \\
& \leq\left|\xi_{1}\right|^{2}|\zeta|^{2}+2<\xi_{1}, \zeta>^{2}+2 \sum_{\beta=1}^{k}<J\left(Y_{\beta}\right) \xi_{1}, \zeta>^{2} .
\end{aligned}
$$

The latter inequality is equivalent to

$$
<\xi_{1}, \zeta>^{2}+\sum_{\beta=1}^{k}<J\left(Y_{\beta}\right) \xi_{1}, \zeta>^{2} \leq\left|\xi_{1}\right|^{2}|\zeta|^{2} .
$$

Now (6.29) is trivially true when $\xi_{1}=0$. If $\xi_{1} \neq 0$, we observe that the set of vectors

$$
\frac{\xi_{1}}{\left|\xi_{1}\right|}, \frac{J\left(Y_{1}\right) \xi_{1}}{\left|\xi_{1}\right|}, \ldots, \frac{J\left(Y_{k}\right) \xi_{1}}{\left|\xi_{1}\right|}
$$


constitutes an orthonormal system in $V_{1}$. This is the only place where we use the structure of Heisenberg type. Orthogonality follows from $<J\left(Y_{\beta}\right) \xi_{1}, \xi_{1}>=0$, and from (6.2) which gives $<J\left(Y_{\beta}\right) \xi_{1}, J\left(Y_{\beta^{\prime}}\right) \xi_{1}>=$ $\delta_{\beta \beta^{\prime}}\left|\xi_{1}\right|^{2}$. From (6.1) we have $\left|J\left(Y_{\beta}\right) \xi_{1}\right|=\left|\xi_{1}\right|$, for every $\beta=1, \ldots, k$. Having observed this, (6.29) is now an immediate consequence of Bessel's inequality. This completes the proof.

Remark 6.7. For the first Heisenberg group $\mathbb{H}^{1}$ the proof of Theorem 6.6 can be simplified to some extent due to the fact that in $\mathbb{R}^{1}$ one has $\langle x, y\rangle=$ $x y$. This causes some special cancellations in the expression of the quadratic form $\left\langle\left(X^{2} N\right)^{\star} \zeta, \zeta>\right.$ which, however, fail to occur in the higher-dimensional case.

Theorem 6.8. Let $N(g)=\left(|x(g)|^{4}+16|y(g)|^{2}\right)^{1 / 4}$ be the gauge in a group of Heisenberg type, then $N \in \mathcal{C}_{H}^{w}(\boldsymbol{G})$.

Proof. We need to prove that given any $g \in \boldsymbol{G}$, for any $g^{\prime} \in H_{g}$ one has for $0 \leq \lambda \leq 1$

$$
N\left(g_{\lambda}\right) \leq(1-\lambda) N(g)+\lambda N\left(g^{\prime}\right)
$$

where as before $g_{\lambda}=g \delta_{\lambda}\left(g^{-1} g^{\prime}\right)$. When $g=e$ we have $g_{\lambda}=\delta_{\lambda} g^{\prime}$, and (6.30) immediately follows from the homogeneity of $N$. Suppose then that $g \neq e$. We distinguish two cases: (a) $g \notin H_{e}$; (b) $g \in H_{e}$. In case (a) we see from (i) in Proposition 4.2 that $e \notin H_{g}$. Therefore, for any $g^{\prime} \in H_{g}$ we also have $g_{\lambda} \neq e$ for $0 \leq \lambda \leq 1$. As a consequence of this observation, if we consider the function $\phi:[0,1] \rightarrow \mathbb{R}$ defined by $\phi(\lambda)=N\left(g_{\lambda}\right)$, we see that $\phi \in C^{2}(0,1)$. We can thus look at $\phi^{\prime \prime}(\lambda)$. From Proposition 5.4, we have

$$
\phi^{\prime \prime}(\lambda)=<\left(X^{2} N\left(g_{\lambda}\right)\right)^{\star} \zeta, \zeta>,
$$

where $\zeta=\xi_{1}\left(g^{-1} g^{\prime}\right)=\xi_{1}\left(g^{\prime}\right)-\xi_{1}(g)$. Thanks to Theorem 6.6, we now know that $\phi^{\prime \prime}(\lambda) \geq 0$, thus concluding that $\phi$ is a convex function on the interval $[0,1]$. This gives in particular $\phi(\lambda) \leq(1-\lambda) \phi(0)+\lambda \phi(1)$, which is $(6.30)$.

We are left with considering case (b). Suppose that $g \in H_{e}$, so that $y(g)=0 \in \mathbb{R}^{k}$. If $g=e$, then $g_{\lambda}=\delta_{\lambda} g^{\prime}$ and again we obtain (6.30) from the homogeneity of $N$. So we can suppose $g \neq e$. Consider $g^{\prime} \in H_{g}$. We distinguish two cases: (i) $g^{\prime}=e$; (ii) $g^{\prime} \neq e$. In case (i) we have $g_{\lambda}=g \delta_{\lambda} g^{-1}$, so that $x\left(g_{\lambda}\right)=(1-\lambda) x(g), y\left(g_{\lambda}\right)=0$, which gives

$$
N\left(g_{\lambda}\right)=(1-\lambda)|x(g)|=(1-\lambda) N(g)+\lambda N(e),
$$


and (6.30) holds. We are thus left with case (ii). If $\xi\left(g^{\prime}\right)$ is not on the straight line segment joining $\xi(g)$ to the origin in $\mathfrak{g}$, then we argue as in case (a) using Theorem 6.6. The final case is when $\xi\left(g^{\prime}\right)$ belongs to the straight line segment joining $\xi(g)$ to the origin in $\mathfrak{g}$, i.e.,

$$
\xi\left(g^{\prime}\right)=a \xi(g)=a \xi_{1}(g),
$$

for some $a \in \mathbb{R}$. The case $a \geq 0$ is easily handled by the previous considerations, so let us assume $a<0$. We then have

$$
x\left(g_{\lambda}\right)=(1-\lambda) x(g)+\lambda(a x(g)), \quad y\left(g_{\lambda}\right)=0,
$$

which gives

$$
\begin{aligned}
N\left(g_{\lambda}\right) & =|(1-\lambda) x(g)+\lambda(a x(g))| \\
& \leq(1-\lambda)|x(g)|+\lambda|a x(g)|=(1-\lambda) N(g)+\lambda N\left(g^{\prime}\right) .
\end{aligned}
$$

This completes the proof.

\section{Convexity of sets.}

In this section we introduce a notion of convexity for sets which turns out to be equivalent, at level of the epigraph, to that of weakly $H$-convex function. Such characterization is very desirable since, quoting from p.25 of [Ro], "...it emphasizes the geometry which is fundamental to the theory of convex functions".

Definition 7.1. A subset $A$ of a Carnot group $\boldsymbol{G}$ is called weakly $H$-convex if for any $g \in A$, and every $g^{\prime} \in A \cap H_{g}$ one has $g_{\lambda}=g_{\lambda}\left(g, g^{\prime}\right) \in A$ for every $\lambda \in[0,1]$. Here, $g_{\lambda}$ is as in (4.1).

Remark 7.2. We remark explicitly that, in view of Proposition 4.4, the geometric interpretation of Definition 7.1 is that for any $g \in A$ the intersection of $A$ with the horizontal plane $H_{g}$ is starlike in the Euclidean sense with respect to $g$ at the level of the Lie algebra.

The next proposition is a simple, yet important, consequence of Definition 7.1 .

Proposition 7.3. A set $A \subset \boldsymbol{G}$ is weakly $H$-convex if and only if such is $g_{o} A$, for every $g_{o} \in \boldsymbol{G}$. 
A basic collection of weakly $H$-convex sets is provided by the next result.

Proposition 7.4. Let $u \in \mathcal{C}_{H}^{w}(\boldsymbol{G})$, then for $a \in \mathbb{R}$ the level sets

$$
\Omega_{a}=\{g \in \boldsymbol{G} \mid u(g) \leq a\},
$$

are weakly $H$-convex. In particular, the gauge balls $B(g, R)$ in a group of Heisenberg type are weakly $H$-convex.

Proof. By the assumption $u \in \mathcal{C}_{H}^{w}(\boldsymbol{G})$ we know for any $g \in \boldsymbol{G}$, and every $g^{\prime} \in H_{g}$

$$
u\left(g_{\lambda}\right) \leq(1-\lambda) u(g)+\lambda u\left(g^{\prime}\right) .
$$

If therefore $g \in \Omega_{a}$, and $g^{\prime} \in \Omega_{a} \cap H_{g}$, we conclude

$$
u\left(g_{\lambda}\right) \leq(1-\lambda) a+\lambda a=a .
$$

The second part now follows from the first and from Theorem 6.8.

We remark explicitly that Proposition 7.4 appears so deceitfully easy because the hard work has already been done in establishing Theorem 6.8.

Remark 7.5. In connection with Remark 7.2 we emphasize that if in Definition 7.1 we substitute the condition " $g$ ' $\in A \cap H_{g}$ " with the stronger one " $g \prime \in A$ ", then the ensuing notion of convexity means unrestricted starlikeness at every point of the set $A$ with respect to the non-isotropic group dilations. Such notion, which is equivalent to that of strongly $H$-convex function in Definition 3.2, is too restrictive, and in fact it fails even for the gauge ball $B(e, 1)$ in the Heisenberg group $\mathbb{H}^{n}$. In other words, the ball $B(e, 1)$ is not strongly $H$-convex.

We next recall the definition of epigraph of a function $u: A \rightarrow \overline{\mathbb{R}}$, where $A \subset G$. This is the set defined by

$$
\text { epi } u=\{(g, s) \in \boldsymbol{G} \times \mathbb{R} \mid u(g) \leq s\} .
$$

In order to characterize the weak $H$-convexity of $u$ in terms of a similar property of its epigraph, we need to endow $\mathcal{G}=\boldsymbol{G} \times \mathbb{R}$ with a corresponding Carnot group structure. This is easily accomplished if we define for $\tilde{g}=$ $(g, s), \tilde{g}^{\prime}=\left(g^{\prime}, s^{\prime}\right) \in \mathcal{G}$

$$
\tilde{g} \tilde{g}^{\prime} \stackrel{\text { def }}{=}\left(g g^{\prime}, s+s^{\prime}\right) \text {. }
$$


The identity in $\mathcal{G}$ is given by $\tilde{e}=(e, 0)$, and for every $\tilde{g}=(g, s)$ we have $\tilde{g}^{-1}=\left(g^{-1},-s\right)$. Denoting by $L_{\tilde{g}} \tilde{g}^{\prime}=\tilde{g} \tilde{g}^{\prime}$ the left-translation on $\mathcal{G}$, one recognizes that in exponential coordinates its differential is represented by the $(N+1) \times(N+1)$ matrix formed by a $N \times N$ block which is just the differential of $L_{g}$ on $\boldsymbol{G}$, and by a remaining row and column in which all the entries are 0 , but the last one which is 1 . The Lie algebra of $\mathcal{G}$ admits a nilpotent stratification of step $r, \tilde{\mathfrak{g}}=\tilde{V}_{1} \oplus \ldots \oplus \tilde{V}_{r}$, where $\tilde{V}_{1}=V_{1} \times \mathbb{R}_{s}$, whereas $\tilde{V}_{j}=V_{j} \times\{0\}_{s}, j=2, \ldots, r$. If $X_{1}, \ldots, X_{m}$ denotes an orthonormal basis of $V_{1}$, then by slightly abusing the notation (in the sense that, although we continue to use the notation $X_{\alpha}$, now this vector field acts on the variable $\tilde{g}=(g, s)$, not just $g)$, we obtain on the group $\mathcal{G}$ the corresponding system of vector fields $\tilde{X}_{\alpha}=X_{\alpha}, \alpha=1, \ldots, m, \tilde{X}_{m+1}=\partial / \partial s$. The natural nonisotropic dilations on $\mathcal{G}$ are defined by

$$
\tilde{\delta}_{\lambda} \tilde{g}=\tilde{\delta}_{\lambda}(g, s) \stackrel{\text { def }}{=}\left(\delta_{\lambda} g, \lambda s\right) .
$$

If we denote by $\tilde{\xi}: \mathcal{G} \rightarrow \tilde{g}=\tilde{\xi}_{1}(\tilde{g})+\ldots+\tilde{\xi}_{r}(\tilde{g})$ the inverse of the exponential mapping defined by $\exp \tilde{\xi}(\tilde{g})=\tilde{g}$, one easily checks that for the projection onto the first layer $\tilde{V}_{1}$ one now has $\tilde{x}_{\alpha}(\tilde{g})=x_{\alpha}(g), \alpha=1, \ldots, m, \tilde{x}_{m+1}(\tilde{g})=$ $s$. From these formulas, it is easy to recognize that the equations of the horizontal plane $H_{\tilde{g}_{o}}$ in $\mathcal{G}$ are unchanged, in the sense that they are the same equations obtained for $H_{g_{o}}$ in $\boldsymbol{G}$, except that now these equations represent an immersed $(m+1)$-dimensional manifold in $\mathcal{G}$. Thus, for instance, if $\boldsymbol{G}=\mathbb{H}^{1}$, the first Heisenberg group, and we have $g_{o}=\left(x_{o}, y_{o}, t_{o}\right) \in \mathbb{H}^{1}$, $s_{o} \in \mathbb{R}, \tilde{g}_{o}=\left(g_{o}, s_{o}\right) \in \mathcal{G}$, then the horizontal plane in the product group $\mathcal{G}$ passing through $\tilde{g}_{o}$ has equation

$$
t=t_{o}+2\left(y_{o} x-x_{o} y\right) .
$$

After these preliminaries we can state the following characterization of weak $H$-convexity.

Proposition 7.6. Given a weakly $H$-convex subset $A \subset \boldsymbol{G}$ of a Carnot group, a function $u: A \rightarrow \mathbb{R}$ is weakly $H$-convex if and only if epi $u$ is a weakly $H$-convex subset of $\mathcal{G}$.

Proof. For every $\lambda \in[0,1]$ consider the point

$$
\tilde{g}_{\lambda}=\tilde{g} \tilde{\delta}_{\lambda}\left(\tilde{g}^{-1} \tilde{g}^{\prime}\right),
$$

where $\tilde{g}=(g, s), \tilde{g}^{\prime}=\left(g^{\prime}, s^{\prime}\right)$. Suppose that $u$ is weakly $H$-convex, and fix $\tilde{g} \in$ epi $u$, so that $u(g) \leq s$. Consider now $\tilde{g}^{\prime} \in$ epi $u \cap H_{\tilde{g}}$, thus in particular 
$u\left(g^{\prime}\right) \leq s^{\prime}$. We want to prove that for every $\lambda \in[0,1]$ one has $\tilde{g}_{\lambda} \in$ epi $u$. From Proposition 4.4 we obtain

$$
\tilde{\xi}\left(\tilde{g}_{\lambda}\right)=\left((1-\lambda) \xi(g)+\lambda \xi\left(g^{\prime}\right),(1-\lambda) s+\lambda s^{\prime}\right) .
$$

This shows that the condition $\tilde{g}_{\lambda} \in$ epi $u$ is equivalent to

$$
u\left(g_{\lambda}\right) \leq(1-\lambda) s+\lambda s^{\prime}
$$

with $g_{\lambda}$ given by (4.1). On the other hand, the weak $H$-convexity of $u$ implies

$$
u\left(g_{\lambda}\right) \leq(1-\lambda) u(g)+\lambda u\left(g^{\prime}\right) \leq(1-\lambda) s+\lambda s^{\prime} .
$$

This proves the necessity. The sufficiency is established by simply reversing the above implications, and choosing $s=u(g), s^{\prime}=u\left(g^{\prime}\right)$ in the end.

\section{Further properties of second derivatives of weakly convex functions.}

In this section we generalize several basic properties of ordinary convex functions to weakly $H$-convex ones on a Carnot group. A classical theorem states that a distribution $T \in \mathcal{D}^{\prime}\left(\mathbb{R}^{N}\right)$ can be identified with a convex function $f$ if and only if its Hessian matrix $\left\{D_{i j} T\right\}$ is a nonnegative $N \times N$ matrix-valued Radon measure $\mu$, see for instance $[R]$, [Du], or [EG]. Our first result establishes a similar property for weakly $H$-convex functions in a Carnot group $G$.

Theorem 8.1. Let $\boldsymbol{G}$ be a Carnot group $\boldsymbol{G}$ and consider $u \in \mathcal{C}_{H}^{w}(\boldsymbol{G}) \cap$ $L_{l o c}^{1}(\boldsymbol{G})$. For $\alpha, \alpha^{\prime}=1, \ldots, m$, there exist signed Radon measures $\nu_{H}^{\alpha \alpha^{\prime}}=\nu_{H}^{\alpha^{\prime} \alpha}$ such that for every $\phi \in C_{o}^{\infty}(\boldsymbol{G})$ one has

$$
\frac{1}{2} \int_{\boldsymbol{G}} u(g)\left\{X_{\alpha} X_{\alpha^{\prime}} \phi(g)+X_{\alpha^{\prime}} X_{\alpha} \phi(g)\right\} d g=\int_{\boldsymbol{G}} \phi(g) d \nu_{H}^{\alpha \alpha^{\prime}}(g) .
$$

In addition, the measures $\nu_{H}^{\alpha \alpha}$ are nonnegative.

Proof. Let $K \in C_{o}^{\infty}(\boldsymbol{G}), K \geq 0$, and consider the corresponding approximation to the identity $\left\{K_{\epsilon}\right\}_{\epsilon>0}$. By Remark 5.9 for every $\epsilon>0$ the function $u_{\epsilon}=K_{\epsilon} \star u \in C^{\infty}(\boldsymbol{G})$ is weakly $H$-convex. Thanks to Theorem 5.12 we obtain for every $\zeta \in V_{1}$, with $|\zeta|=1$

$$
\int_{\boldsymbol{G}} u_{\epsilon}(g)<\left(X^{2} \phi(g)\right)^{\star} \zeta, \zeta>d g=\int_{\boldsymbol{G}} \phi(g)<\left(X^{2} u_{\epsilon}(g)\right)^{\star} \zeta, \zeta>d g \geq 0 .
$$


Passing to the limit as $\epsilon \rightarrow 0$ we find

$$
T_{\zeta}(\phi) \stackrel{\text { def }}{=} \int_{\boldsymbol{G}} u(g)<\left(X^{2} \phi(g)\right)^{\star} \zeta, \zeta>d g \geq 0
$$

which shows that $T_{\zeta}: C_{o}^{\infty}(\boldsymbol{G}) \rightarrow \mathbb{R}$ defines a nonnegative linear functional. By the Riesz representation theorem there exists a nonnegative Radon measure $\nu_{H}^{\zeta}$ such that

$$
T_{\zeta}(\phi)=\int_{\boldsymbol{G}} \phi(g) d \nu_{H}^{\zeta}(g), \quad \phi \in C_{o}^{\infty}(\boldsymbol{G})
$$

Recalling that $\left\{X_{1}, \ldots, X_{m}\right\}$ is an orthonormal basis of $V_{1}$, we now define

$$
\nu_{H}^{\alpha \alpha}=\nu_{H}^{X_{\alpha}},
$$

and observe that if $\alpha \neq \alpha^{\prime}$ the vector

$$
\zeta_{\alpha \alpha^{\prime}} \stackrel{\text { def }}{=} \frac{X_{\alpha}+X_{\alpha^{\prime}}}{\sqrt{2}} \in V_{1}
$$

is such that $\left|\zeta_{\alpha \alpha^{\prime}}\right|=1$. With this choice we have

$$
\begin{aligned}
& <\left(X^{2} \phi(g)\right)^{\star} \zeta_{\alpha \alpha^{\prime}}, \zeta_{\alpha \alpha^{\prime}}> \\
& =\frac{1}{2}\left\{X_{\alpha} X_{\alpha} \phi(g)+X_{\alpha} X_{\alpha^{\prime}} \phi(g)+X_{\alpha^{\prime}} X_{\alpha} \phi(g)+X_{\alpha^{\prime}} X_{\alpha^{\prime}} \phi(g)\right\},
\end{aligned}
$$

which gives

$$
\begin{aligned}
& \int_{\boldsymbol{G}} \phi(g) d \nu_{H}^{\zeta_{\alpha \alpha^{\prime}}}(g)=\frac{1}{2} \int_{\boldsymbol{G}} \phi(g) d \nu_{H}^{\alpha \alpha}(g) \\
& +\frac{1}{2} \int_{\boldsymbol{G}} u(g)\left\{X_{\alpha} X_{\alpha^{\prime}} \phi(g)+X_{\alpha^{\prime}} X_{\alpha} \phi(g)\right\} d g+\frac{1}{2} \int_{\boldsymbol{G}} \phi(g) d \nu_{H}^{\alpha^{\prime} \alpha^{\prime}}(g) .
\end{aligned}
$$

If we define the signed Radon measure

$$
\nu_{H}^{\alpha \alpha^{\prime}} \stackrel{\text { def }}{=} \nu_{H}^{\frac{X_{\alpha}+X_{\alpha^{\prime}}}{\sqrt{2}}}-\frac{1}{2} \nu_{H}^{\alpha \alpha}-\frac{1}{2} \nu_{H}^{\alpha^{\prime} \alpha^{\prime}}
$$

then from (8.1) we reach the conclusion

$$
\frac{1}{2} \int_{G} u(g)\left\{X_{\alpha} X_{\alpha^{\prime}} \phi(g)+X_{\alpha^{\prime}} X_{\alpha} \phi(g)\right\} d g=\int_{G} \phi(g) d \nu_{H}^{\alpha \alpha^{\prime}}(g) .
$$

This completes the proof. 
Remark 8.2. A classical consequence of the Euclidean counterpart of Theorem 8.1 is that the first derivatives of a convex function $f: \mathbb{R}^{N} \rightarrow \mathbb{R}$ are locally in $B V$, the space of functions with bounded variation. This result was first proved by Bakelman [Ba2]. It would be desirable to have a similar result for a weakly $H$-convex function $u$ on a Carnot group, but this does not seem to follow directly from Theorem 8.1. The main obstruction being that the representability of $X_{\alpha} X_{\alpha^{\prime}} u+X_{\alpha^{\prime}} X_{\alpha} u$ by a Radon measure does not directly guarantee a similar property for $X_{\alpha} X_{\alpha^{\prime}} u$ and $X_{\alpha^{\prime}} X_{\alpha} u$. This is of course a reflection of the lack of commutation $X_{\alpha} X_{\alpha^{\prime}} u \neq X_{\alpha^{\prime}} X_{\alpha} u$.

In some cases it is possible to prove that the second horizontal derivatives $X_{\alpha} X_{\alpha^{\prime}} u$ are Radon measures. This follows from the following elementary proposition.

Proposition 8.3. Let $u \in L_{l o c}^{1}(\boldsymbol{G})$ be a weakly $H$-convex function in a Carnot group $\boldsymbol{G}$. If for every $\alpha, \alpha^{\prime}=1, \ldots, m$, the commutators $\left[X_{\alpha}, X_{\alpha^{\prime}}\right] u$ are Radon measures, then such are also $X_{\alpha} X_{\alpha^{\prime}} u$.

Proof. One has

$$
X_{\alpha} X_{\alpha^{\prime}} u=\frac{1}{2}\left\{X_{\alpha} X_{\alpha^{\prime}} u+X_{\alpha^{\prime}} X_{\alpha} u\right\}+\frac{1}{2}\left[X_{\alpha}, X_{\alpha^{\prime}}\right] u .
$$

Since from Theorem 8.1 we know that $\frac{1}{2}\left\{X_{\alpha} X_{\alpha^{\prime}} u+X_{\alpha^{\prime}} X_{\alpha} u\right\}$ is a Radon measure, the conclusion follows.

Corollary 8.1. Under the assumptions of Proposition 8.3, suppose in addition that $X_{\alpha} u \in L_{l o c}^{1}(\boldsymbol{G})$, for $\alpha=1, \ldots, m$. One then has $u \in B V_{H, l o c}^{2}(\boldsymbol{G})$. Here, $B V_{H, l o c}^{2}(\boldsymbol{G})$ denotes the space of functions $u \in \mathcal{L}_{\text {loc }}^{1,1}(\Omega)$ whose derivatives $X_{\alpha} u, \alpha=1, \ldots, m$, are locally of horizontal bounded variation.

Proof. We recall the notion of horizontal bounded variation introduced in [CDG] and subsequently developed in [GN1]. Let $\Omega \subset \boldsymbol{G}$ be an open set, and $u \in L_{l o c}^{1}(\Omega)$. Denote by $\zeta=\sum_{\alpha=1}^{m} \zeta_{\alpha} X_{\alpha}$ an element of $C_{o}^{1}(\Omega ; H \Omega)$. Let

$$
\mathcal{F}_{H}(\Omega)=\left\{\zeta \in C_{o}^{1}(\Omega ; H \Omega) \mid\|\zeta\|_{\infty} \leq 1\right\} .
$$

The $H$-variation of $u$ in $\Omega$ is defined as follows

$$
\operatorname{Var}_{H}(u ; \Omega)=\sup _{\zeta \in \mathcal{F}_{H}(\Omega)} \int_{\Omega} u \sum_{\alpha=1}^{m} X_{\alpha} \zeta_{\alpha} d g .
$$


A function $u \in L^{1}(\Omega)$ is called of bounded $H$-variation if $\operatorname{Var}_{H}(u ; \Omega)<$ $\infty$. In such case, we write $u \in B V_{H}(\Omega)$, and the collection of all such functions becomes a Banach space when endowed with the norm

$$
\|u\|_{B V_{H}(\Omega)}=\|u\|_{L^{1}(\Omega)}+\operatorname{Var}_{H}(u ; \Omega) .
$$

The notation $B V_{H, l o c}(\Omega)$ indicates the collection of functions $u \in L_{l o c}^{1}(\Omega)$, such that $u \in B V_{H}(\omega)$, for every $\omega \subset \subset \Omega$.

Returning to the proof of the corollary, let then $\omega \subset \subset \boldsymbol{G}$, and consider $\zeta \in \mathcal{F}_{H}(\omega)$. For any $\alpha=1, \ldots, m$ we have

$$
\int_{\omega} X_{\alpha} u \sum_{\alpha^{\prime}=1}^{m} X_{\alpha^{\prime}} \zeta_{\alpha^{\prime}} d g=-\sum_{\alpha^{\prime}=1}^{m} \int_{\omega} u X_{\alpha} X_{\alpha^{\prime}} \zeta_{\alpha^{\prime}} d g
$$

Using Theorem 8.1 we obtain

$\int_{\omega} X_{\alpha} u \sum_{\alpha^{\prime}=1}^{m} X_{\alpha^{\prime}} \zeta_{\alpha^{\prime}} d g=-2 \sum_{\alpha^{\prime}=1}^{m} \int_{G} \zeta_{\alpha^{\prime}}(g) d \nu_{H}^{\alpha \alpha^{\prime}}(g)+\sum_{\alpha^{\prime}=1}^{m}\left(X_{\alpha} X_{\alpha^{\prime}} u, \zeta_{\alpha^{\prime}}\right)$,

where we have denoted by $(\cdot, \cdot)$ the duality between $\mathcal{D}^{\prime}(\boldsymbol{G})$ and $\mathcal{D}(\boldsymbol{G})$. By Proposition 8.3 we know that also $X_{\alpha} X_{\alpha^{\prime}} u$ are Radon measures, therefore we conclude

$$
\int_{\omega} X_{\alpha} u \sum_{\alpha^{\prime}=1}^{m} X_{\alpha^{\prime}} \zeta_{\alpha^{\prime}} d g \leq 2 \sum_{\alpha^{\prime}=1}^{m} \nu_{H}^{\alpha \alpha^{\prime}}(\omega)+\sum_{\alpha^{\prime}=1}^{m} X_{\alpha} X_{\alpha^{\prime}} u(\omega)<\infty .
$$

Taking the supremum on all $\zeta \in \mathcal{F}_{H}(\omega)$ we reach the conclusion.

An interesting consequence of the above results and of Theorem 6.8 is provided by the following proposition which finds applications in optimal control.

Proposition 8.4. Let $\boldsymbol{G}$ be a Carnot group of step 2 with non-isotropic gauge $N$, then the commutators $\left[X_{\alpha}, X_{\alpha^{\prime}}\right] N$ belong to $L_{l o c}^{1}(\boldsymbol{G})$. When $\boldsymbol{G}$ is of Heisenberg type $\boldsymbol{G}$, we obtain

$$
N^{p} \in B V_{H, l o c}^{2}(\boldsymbol{G}), \quad \text { for every } p \geq 1 \text {. }
$$

Proof. For convenience we consider the gauge renormalized by the (immaterial) factor 16, i.e., $N(g)=\left(|x(g)|^{4}+16|y(g)|^{2}\right)^{1 / 4}$. Formula (5.10) gives 
for $g \in \boldsymbol{G} \backslash\{e\}$

$$
\begin{aligned}
{\left[X_{\alpha}, X_{\alpha^{\prime}}\right] N(g) } & =\sum_{\beta=1}^{k} b_{\alpha \alpha^{\prime}}^{\beta} \frac{\partial N}{\partial y_{\beta}} \\
& =\frac{8}{N(g)^{3}} \sum_{\beta=1}^{k} b_{\alpha \alpha^{\prime}}^{\beta} y_{\beta}(g) .
\end{aligned}
$$

The trivial observation that $\left|4 y_{\beta}(g)\right| \leq N(g)^{2}$ allows to conclude from $(8.2)$

$$
\left|\left[X_{\alpha}, X_{\alpha^{\prime}}\right] N(g)\right| \leq \frac{C_{\alpha \alpha^{\prime}}}{N(g)}
$$

where we have let $C_{\alpha \alpha^{\prime}}=2 \sum_{\beta=1}^{k}\left|b_{\alpha \alpha^{\prime}}^{\beta}\right|$. Since $N^{-1} \in L_{l o c}^{q}(\boldsymbol{G})$ for every $1 \leq q<Q$, where $Q$ represents the homogeneous dimension of $\boldsymbol{G}$, the first part of the proposition follows.

Suppose now that $\boldsymbol{G}$ be of Heisenberg type, then by Theorem 6.8 we know that $N$ is weakly $H$-convex. Furthermore, by (6.11) in Lemma 6.3 we have

$$
|X N(g)|^{2}=\frac{|x(g)|^{2}}{N(g)^{2}} \leq 1
$$

and therefore $N \in \mathcal{L}_{l o c}^{1, \infty}(\boldsymbol{G})$. All the hypothesis of Proposition 8.3, and therefore of Corollary 8.1, are thus fulfilled. The conclusion follows.

Remark 8.5. In connection with Remark 8.2, we mention that we have recently received an interesting preprint from $L$. Ambrosio and $V$. Magnani $[A M]$ in which the authors prove, among other things, that if $u \in B V_{H}^{2}(\boldsymbol{G})$, then for $d g$-a.e. $g_{o} \in \boldsymbol{G}$ there exists a polynomial $P\left(g_{o} ; \cdot\right)$ of weighted degree $\leq 2$, such that

$$
\lim _{r \rightarrow 0} \frac{1}{r^{2}} \frac{1}{\left|B\left(g_{o}, r\right)\right|} \int_{B\left(g_{o}, r\right)}\left|u(g)-P\left(g_{o}, g\right)\right| d g=0 .
$$

In the classical setting, as a consequence of Bakelman's result cited in Remark 8.2, the class $B V_{l o c}^{2}$ contains that of convex functions, therefore (8.3) can be viewed as an integral version for Carnot groups of the Theorem of Busemann-Feller $(n=2)$ and Alexandrov $(n \geq 3)$ on the existence a.e. of second derivatives of a convex function. However, (8.3) leaves open the question of the actual sub-Riemannian pointwise version of the BusemannFeller-Alexandrov theorem for convex functions. For functions which are in $B V_{H}^{2}(\boldsymbol{G}) \cap C_{H}^{w}(\boldsymbol{G}) \cap C(\boldsymbol{G})$ such result can be obtained as a consequence of 
our Theorem 9.2 in the next section, and of (8.3). We plan to come back to these questions in a forthcoming article.

\section{Basic properties of first derivatives of weakly convex} functions: $L^{\infty}-L^{1}$ estimates.

An essential property of convex functions is that they are locally Lipschitz continuous. This section is devoted to proving some basic quantitative versions for continuous weakly $H$-convex functions in a Carnot group $\boldsymbol{G}$. The main result is Theorem 9.2. A remarkable aspect of the latter is that from an information on lower dimensional manifolds (weak $H$-convexity), it draws a conclusion on a set of full measure, see (9.7), (9.8). We mention that if, instead of assuming continuity, we only suppose that the function $u$ belong to $L_{l o c}^{\infty}(\boldsymbol{G})$, then we can still conclude membership in $\Gamma_{l o c}^{0,1}(\boldsymbol{G})$, but of course after redefinition on a set of measure zero.

Theorem 9.1. Let $u \in \mathcal{C}_{H}^{w}(\boldsymbol{G}) \cap C(\boldsymbol{G})$ in a Carnot group $\boldsymbol{G}$, then $u \in$ $\Gamma_{\text {loc }}^{0,1}(\boldsymbol{G})$. Furthermore, there exists $C=C(\boldsymbol{G})>0$ such that the following estimate holds

$$
\|X u\|_{L^{\infty}\left(B\left(g_{o}, R\right)\right)} \leq \frac{C}{R}\|u\|_{L^{\infty}\left(B\left(g_{o}, 3 R\right)\right)}
$$

for any $g_{o} \in \boldsymbol{G}$ and every $R>0$. In particular, (9.1) shows that there exist no bounded functions in $\mathcal{C}_{H}^{w}(\boldsymbol{G}) \cap C(\boldsymbol{G})$, other than the constant functions.

Proof. Given a kernel $K \in C_{o}^{\infty}(\boldsymbol{G}), K \geq 0$, supp $K \subset \bar{B}(e, 1)$, $\int_{G} K(g) d g=1$, consider the corresponding approximation to the identity $\left\{K_{\epsilon}\right\}_{\epsilon>0}$ associated with it. Let $u_{\epsilon}=K_{\epsilon} \star u$, then $u_{\epsilon} \in C^{\infty}(\boldsymbol{G})$, and by the hypothesis $u \in C(\boldsymbol{G})$ we have $u_{\epsilon} \rightarrow u$ uniformly on compact subsets of $\boldsymbol{G}$.

We now fix a gauge ball $B\left(g_{o}, R\right) \subset B\left(g_{o}, 3 R\right) \subset \boldsymbol{G}$. By Remark 5.9 the functions $u_{\epsilon}$ are weakly $H$-convex. Proposition 5.7 implies for any fixed $g \in B\left(g_{o}, R\right)$ and every $g^{\prime} \in H_{g}$

$$
<X u_{\epsilon}(g), x\left(g^{\prime}\right)-x(g)>\leq u_{\epsilon}\left(g^{\prime}\right)-u_{\epsilon}(g) \text {. }
$$

For $g^{\prime} \in H_{g} \backslash\{g\}$ we rewrite the latter inequality as follows

$$
(9.2)<X u_{\epsilon}(g), \frac{x\left(g^{\prime}\right)-x(g)}{\left|x\left(g^{\prime}\right)-x(g)\right|}>\leq \frac{u_{\epsilon}\left(g^{\prime}\right)-u_{\epsilon}(g)}{\left|x\left(g^{\prime}\right)-x(g)\right|}=\frac{u_{\epsilon}\left(g^{\prime}\right)-u_{\epsilon}(g)}{d\left(g^{\prime}, g\right)},
$$


where we have used the fact that when $g^{\prime} \in H_{g}$, then

$$
d\left(g^{\prime}, g\right)=\left|x\left(g^{\prime}\right)-x(g)\right| .
$$

This can be easily seen as follows. The condition $g^{\prime} \in H_{g}$ is equivalent to saying that $g^{\prime}=g h$, with $h \in H_{e}$. Therefore, we obtain from (2.12), (2.11) and $(2.10)$

$$
d\left(g^{\prime}, g\right)=N\left(g^{-1} g^{\prime}\right)=N(h)=|x(h)|,
$$

the latter equality being justified by the fact $\xi_{2}(h)=0, \ldots, \xi_{r}(h)=0$. On the other hand, (2.7) gives $x(h)=x\left(g^{-1} g^{\prime}\right)=x\left(g^{\prime}\right)-x(g)$, and (9.3) follows. If we now take $g^{\prime} \in \partial B(g, R) \cap H_{g}$ in (9.2), for $0<\epsilon<R / 2$ we obtain

$$
\begin{aligned}
<X u_{\epsilon}(g), \frac{x\left(g^{\prime}\right)-x(g)}{\left|x\left(g^{\prime}\right)-x(g)\right|}> & \leq \frac{u_{\epsilon}\left(g^{\prime}\right)-u_{\epsilon}(g)}{R} \\
& \leq \frac{2}{R}\left\|u_{\epsilon}\right\|_{L^{\infty}\left(B\left(g_{o}, 2 R\right)\right)} \leq \frac{2}{R}\|u\|_{L^{\infty}\left(B\left(g_{o}, 3 R\right)\right)} .
\end{aligned}
$$

Passing to the supremum on all $g^{\prime} \in \partial B(g, R) \cap H_{g}$ we find

$$
\left|X u_{\epsilon}(g)\right| \leq \frac{2}{R}\|u\|_{L^{\infty}\left(B\left(g_{o}, 3 R\right)\right)} .
$$

By the arbitrariness of $g \in B\left(g_{o}, R\right)$ we conclude

$$
\left\|X u_{\epsilon}\right\|_{L^{\infty}\left(B\left(g_{o}, R\right)\right)} \leq \frac{2}{R}\|u\|_{L^{\infty}\left(B\left(g_{o}, 3 R\right)\right)} .
$$

From Theorem 2.5, and from (9.5) we obtain

$$
\left|u_{\epsilon}(g)-u_{\epsilon}\left(g^{\prime}\right)\right| \leq \frac{C}{R}\|u\|_{L^{\infty}\left(B\left(g_{o}, 3 R\right)\right)} d\left(g, g^{\prime}\right), \quad g, g^{\prime} \in \bar{B}\left(g_{o}, R\right),
$$

where $C>0$ is an absolute constant. Passing to the limit as $\epsilon \rightarrow 0$ we find

$$
\left|u(g)-u\left(g^{\prime}\right)\right| \leq \frac{C}{R}\|u\|_{L^{\infty}\left(B\left(g_{o}, 3 R\right)\right)} d\left(g, g^{\prime}\right), \quad g, g^{\prime} \in B\left(g_{o}, R\right) .
$$

This shows that the function $u$ belongs to $\Gamma_{l o c}^{0,1}(\boldsymbol{G})$. At this point using Theorem 2.4 we conclude from (9.6) that the weak derivatives $X_{1} u, \ldots, X_{m} u$ exist $d g$-a.e. in $\boldsymbol{G}$, and they belong to $L_{l o c}^{\infty}\left(\mathbb{H}^{n}\right)$. Moreover, we obtain the bound

$$
\|X u\|_{L^{\infty}\left(B\left(g_{o}, R\right)\right)} \leq \frac{C}{R}\|u\|_{L^{\infty}\left(B\left(g_{o}, 3 R\right)\right)},
$$


which proves (9.1), thus completing the proof.

We next establish an important improvement of Theorem 9.1 in which we replace the $L^{\infty}$ norm in the right hand side of (9.1) with the $L^{1}$ average of the relevant function.

Theorem 9.2. Let $u \in \mathcal{C}_{H}^{w}(\boldsymbol{G}) \cap C(\boldsymbol{G})$, then there exists $C=C(\boldsymbol{G})>0$ such that for every ball $B(g, r)$ one has

$$
\sup _{B(g, r)}|u| \leq C \frac{1}{|B(g, 5 r)|} \int_{B(g, 5 r)}|u| d g^{\prime},
$$

and

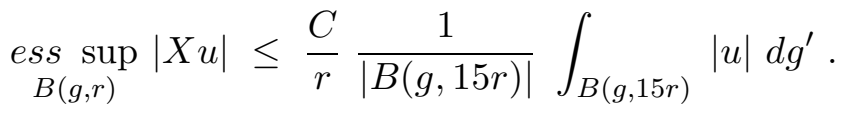

In particular, (9.7) implies that there exist no $L^{1}$ functions in $\mathcal{C}_{H}^{w}(\boldsymbol{G}) \cap C(\boldsymbol{G})$ other than the trivial one.

Proof. We begin by remarking that, thanks to the estimate (9.1), in order to prove the theorem we only need to establish (9.7). Similarly to the proof of Theorem 9.1 it is not restrictive to assume that $u \in C^{\infty}(\boldsymbol{G})$, since we can always work with the smooth weakly $H$-convex functions $u_{\epsilon}=K_{\epsilon} \star u$, and then pass to the limit. We observe that $u$ is in $\mathcal{C}_{H}^{w}(\boldsymbol{G})$ if and only if for any $r>0$ the rescaled function $u_{r}(g)=u\left(\delta_{r} g\right)$ belongs to $\mathcal{C}_{H}^{w}(\boldsymbol{G})$. This follows from the following equation

$$
\delta_{r}\left(g \delta_{\lambda}\left(g^{-1} g^{\prime}\right)\right)=\left(\delta_{r} g\right)\left(\delta_{r}\left(\delta_{\lambda}\left(g^{-1} g^{\prime}\right)\right)=\left(\delta_{r} g\right) \delta_{\lambda}\left(\left(\delta_{r} g\right)^{-1} \delta_{r} g^{\prime}\right),\right.
$$

and from the observation that $g^{\prime} \in H_{g}$ if and only if $\delta_{r} g^{\prime} \in H_{\delta_{r} g}$. It therefore suffices to prove (9.7) when $r=1$. At this point to simplify the technical details we present a detailed account of the proof in the case of the first Heisenberg group $\mathbb{H}^{1}$. At the end, we briefly indicate the minor adjustments necessary to treat the general case. Let then $g_{o} \in B(g, 1)$ be such that $u\left(g_{o}\right)=\sup _{B(g, 1)} u$. By left-translation we can suppose that $g_{o}=e$. Consider the straight line segment $\gamma(s)=(s, 0,0),-1 \leq s \leq 1$, and observe that such segment lies on $H_{e}$. We define the function $\phi:[-1,1] \rightarrow \mathbb{R}$ by the equation $\phi(s)=u(\gamma(s))$. The smoothness of $u$ implies that $\phi \in C^{\infty}$. A simple computation gives $\phi^{\prime \prime}(s)=X_{1} X_{1} u(s)$. Applying Theorem 5.11 we 
now conclude that $\phi^{\prime \prime}(s) \geq 0$ for every $s \in[-1,1]$, therefore $\phi$ is a convex function on the interval $[-1,1]$. Jensen's inequality then implies

$$
\phi(0) \leq \frac{1}{2} \int_{-1}^{1} \phi(s) d s
$$

which we rewrite

$$
u(e) \leq \frac{1}{2} \int_{-1}^{1} u(\gamma(s)) d s
$$

For any $s \in[-1,1]$, we apply Proposition 5.7 obtaining for every $g^{\prime} \in$ $H_{\gamma(s)}$

$$
u(\gamma(s)) \leq u\left(g^{\prime}\right)-<X u(\gamma(s)), x\left(g^{\prime}\right)-x(\gamma(s))>.
$$

We integrate the latter inequality with respect to $g^{\prime}$ and to 2-dimensional Lebesgue measure $d \sigma\left(g^{\prime}\right)$ on the set $B(\gamma(s), 1) \cap H_{\gamma(s)}$, obtaining

$$
\begin{aligned}
& u(\gamma(s)) \int_{B(\gamma(s), 1) \cap H_{\gamma(s)}} d \sigma\left(g^{\prime}\right) \leq \int_{B(\gamma(s), 1) \cap H_{\gamma(s)}} u\left(g^{\prime}\right) d \sigma\left(g^{\prime}\right) \\
& -\int_{B(\gamma(s), 1) \cap H_{\gamma(s)}}<X u(\gamma(s)), x\left(g^{\prime}\right)-x(\gamma(s))>d \sigma\left(g^{\prime}\right) .
\end{aligned}
$$

A simple computation gives

$$
\int_{B(\gamma(s), 1) \cap H_{\gamma(s)}} d \sigma\left(g^{\prime}\right)=\pi \sqrt{1+4 s^{2}},
$$

whereas

$$
\begin{aligned}
& \int_{B(\gamma(s), 1) \cap H_{\gamma(s)}}<X u(\gamma(s)), x\left(g^{\prime}\right)-x(\gamma(s))>d \sigma\left(g^{\prime}\right) \\
& =\sqrt{1+4 s^{2}} \int_{\left(x^{\prime}-s\right)^{2}+\left(y^{\prime}\right)^{2}<1}\left\{X_{1} u(\gamma(s))\left(x^{\prime}-s\right)+X_{2} u(\gamma(s)) y^{\prime}\right\} d x^{\prime} d y^{\prime} \\
& =\sqrt{1+4 s^{2}} \int_{x^{2}+y^{2}<1}\left\{X_{1} u(\gamma(s)) x+X_{2} u(\gamma(s)) y\right\} d x d y=0
\end{aligned}
$$

the vanishing of the latter integral being justified by the fact that the integrand is an odd function of the variable of integration $(x, y)$. Inserting these two equations in (9.10) we conclude

$$
u(\gamma(s)) \leq \frac{1}{\pi} \int_{B(\gamma(s), 1) \cap H_{\gamma(s)}}\left|u\left(g^{\prime}\right)\right| d \sigma\left(g^{\prime}\right) .
$$


At this point we integrate in $s \in(-1,1)$ the inequality (9.11) and obtain

$$
\int_{-1}^{1} u(\gamma(s)) d s \leq \frac{1}{\pi} \int_{-1}^{1} \int_{B(\gamma(s), 1) \cap H_{\gamma(s)}}\left|u\left(g^{\prime}\right)\right| d \sigma\left(g^{\prime}\right) .
$$

From (9.12), and from (9.9), we conclude

$$
u(e) \leq \frac{1}{2 \pi} \int_{-1}^{1} \int_{B(\gamma(s), 1) \cap H_{\gamma(s)}}\left|u\left(g^{\prime}\right)\right| d \sigma\left(g^{\prime}\right) d s .
$$

We now observe that the integral in the right-hand side of the latter inequality is finite. To see this we notice that as $s$ varies in $[-1,1]$ the regions $B(\gamma(s), 1) \cap H_{\gamma(s)}$ overlap only along the segment of the $x$-axis (which has 2 -dimensional measure zero) joining the points $(-1,0,0),(1,0,0)$. Furthermore, one easily sees that

$$
B(\gamma(s), 1) \cap H_{\gamma(s)} \subset W_{s}=\{(x, y, t)|| x|<2,| y \mid<1, t=-2 s y\}
$$

and

$$
\bigcup_{s \in[-1,1]} W_{s} \subset B(e, 4)
$$

From (9.14), (9.15), and from (9.13), we conclude

$$
\sup _{B(g, 1)} u=u(e) \leq C_{1} \int_{B(e, 4)}\left|u\left(g^{\prime}\right)\right| d g^{\prime} \leq C_{1} \int_{B(g, 5)}\left|u\left(g^{\prime}\right)\right| d g^{\prime} .
$$

This proves that $u$ is bounded from above in $L^{1}$ norm.

To establish an analogous bound from below, we argue as follows. Let $g_{1} \in B(g, 1)$ be such that $u\left(g_{1}\right)=\inf _{B(g, 1)} u$. As before, by left-translation we can assume that $g_{1}=e$. Let $g^{\prime} \in B(e, 1) \cap H_{e}$ be fixed. By Proposition 4.2 we have $e \in H_{g^{\prime}}$, and therefore Proposition 5.7 allows to conclude

$$
u(e) \geq u\left(g^{\prime}\right)-<X u\left(g^{\prime}\right), x\left(g^{\prime}\right)>.
$$

We choose $\zeta \in C_{o}^{\infty}(B(e, 1)), 0 \leq \zeta \leq 1, \zeta \equiv 1$ on $B(e, 1 / 2)$, multiply (9.17) by $\zeta\left(g^{\prime}\right)$, and integrate with respect to $d \sigma\left(g^{\prime}\right)$ over $B(e, 1) \cap H_{e}$ to obtain

$$
\begin{aligned}
& u(e) \int_{B(e, 1) \cap H_{e}} \zeta\left(g^{\prime}\right) d \sigma\left(g^{\prime}\right) \\
& \geq \int_{B(e, 1) \cap H_{e}} \zeta\left(g^{\prime}\right) u\left(g^{\prime}\right) d \sigma\left(g^{\prime}\right)-\int_{B(e, 1) \cap H_{e}}<X u\left(g^{\prime}\right), x\left(g^{\prime}\right)>\zeta\left(g^{\prime}\right) d \sigma\left(g^{\prime}\right) .
\end{aligned}
$$


At this point, an important cancellation occurs in the integral involving $X u$

$$
\begin{aligned}
& \int_{B(e, 1) \cap H_{e}}<X u\left(g^{\prime}\right), x\left(g^{\prime}\right)>\zeta\left(g^{\prime}\right) d \sigma\left(g^{\prime}\right) \\
& =\int_{B(e, 1) \cap H_{e}}\left\{X_{1} u\left(g^{\prime}\right) x^{\prime}+X_{2} u\left(g^{\prime}\right) y^{\prime}\right\} \zeta\left(g^{\prime}\right) d \sigma\left(g^{\prime}\right) \\
& =\int_{B(e, 1) \cap H_{e}}\left\{\left[u_{x}\left(g^{\prime}\right)+2 y^{\prime} u_{t}\left(g^{\prime}\right)\right] x^{\prime}+\left[u_{y}\left(g^{\prime}\right)-2 x^{\prime} u_{t}\left(g^{\prime}\right)\right] y^{\prime}\right\} \zeta\left(g^{\prime}\right) d \sigma\left(g^{\prime}\right) \\
& =\int_{B(e, 1) \cap H_{e}}\left\{x^{\prime} u_{x}\left(g^{\prime}\right)+y^{\prime} u_{y}\left(g^{\prime}\right)\right\} \zeta\left(g^{\prime}\right) d \sigma\left(g^{\prime}\right) \\
& =-\int_{B(e, 1) \cap H_{e}}\left\{\left(x^{\prime} \zeta\right)_{x^{\prime}}+\left(y^{\prime} \zeta\right)_{y^{\prime}}\right\} u\left(g^{\prime}\right) d \sigma\left(g^{\prime}\right),
\end{aligned}
$$

where we notice that when integrating by parts the boundary integral on $\partial B(e, 1) \cap H_{e}$ vanishes due to our choice of $\zeta$. From the latter equation, the inequality

$$
\sigma\left(B(e, 1 / 2) \cap H_{e}\right) \leq \int_{B(e, 1) \cap H_{e}} \zeta\left(g^{\prime}\right) d \sigma\left(g^{\prime}\right) \leq \sigma\left(B(e, 1) \cap H_{e}\right),
$$

and from (9.18), we conclude the existence of a constant $C_{2}>0$ such that

$$
u(e) \geq-C_{2} \int_{B(e, 1) \cap H_{e}}\left|u\left(g^{\prime}\right)\right| d \sigma\left(g^{\prime}\right) .
$$

We now assert that an estimate similar to (9.19) holds at any point $\gamma(s)$, $s \in[-1,1]$, with an absolute constant $C_{2}>0$ independent of $s$, that is

$$
u(\gamma(s)) \geq-C_{2} \int_{B(\gamma(s), 1) \cap H_{\gamma(s)}}\left|u\left(g^{\prime}\right)\right| d \sigma\left(g^{\prime}\right) .
$$

The details of the verification of (9.20) are similar to those in the proof of (9.19) and are left to the reader. If we now let, as in the proof of (9.16), $\phi(s)=u(\gamma(s))$, then we can rewrite (9.20) as follows

$$
\phi(s) \geq-C_{2} \int_{B(\gamma(s), 1) \cap H_{\gamma(s)}}\left|u\left(g^{\prime}\right)\right| d \sigma\left(g^{\prime}\right), \quad-1 \leq s \leq 1 .
$$

Integrating (9.21) on the interval [0,1], and using (9.14), (9.15), we obtain

$$
\int_{0}^{1} \phi(s) d s \geq-C_{2} \int_{B(e, 4)}\left|u\left(g^{\prime}\right)\right| d g^{\prime} \geq-C_{2} \int_{B(g, 5)}\left|u\left(g^{\prime}\right)\right| d g^{\prime}
$$


On the other hand, the convexity of $\phi$ on $[0,1]$ gives

$$
\phi(s) \leq(1-s) \phi(0)+s \phi(1), \quad 0 \leq s \leq 1 .
$$

Integrating the latter inequality on $[0,1]$ one finds

$$
\int_{0}^{1} \phi(s) d s \leq \frac{1}{2} \phi(0)+\frac{1}{2} \phi(1)=\frac{1}{2} u(e)+\frac{1}{2} u\left(g_{o}\right),
$$

where we have let $g_{o}=(1,0,0) \in \bar{B}(e, 1)$. Invoking (9.16), in which we take $g=e$, we obtain

$$
u\left(g_{o}\right) \leq \sup _{\bar{B}(e, 1)} u \leq C_{1} \int_{B(e, 4)}\left|u\left(g^{\prime}\right)\right| d g^{\prime} \leq C_{1} \int_{B(g, 5)}\left|u\left(g^{\prime}\right)\right| d g^{\prime} .
$$

Inserting this estimate in the previous inequality we conclude

$$
\int_{0}^{1} \phi(s) d s \leq \frac{1}{2} u(e)+\frac{C_{1}}{2} \int_{B(g, 5)}\left|u\left(g^{\prime}\right)\right| d g^{\prime} .
$$

Finally, the inequalities (9.22), (9.23) imply, with $C=\max \left\{C_{1}, C_{2}\right\}$,

$$
\inf _{B(g, 1)} u=u(e) \geq-3 C \int_{B(g, 5)}\left|u\left(g^{\prime}\right)\right| d g^{\prime} .
$$

This establishes the sought for estimate from below, which, together with (9.16), proves (9.7) when $\boldsymbol{G}=\mathbb{H}^{1}$. In the case of a general Carnot group $\boldsymbol{G}$ we define $\gamma:[-1,1] \rightarrow \boldsymbol{G}$ as follows

$$
\gamma(s)=\exp \left(s X_{1}\right)
$$

where as always $X_{1}$ denotes the first element of a fixed orthonormal basis of the first layer $V_{1}$ of $\mathfrak{g}$. We note explicitly that the trace of the curve $\gamma$ lies in the horizontal plane $H_{e}$. With this choice of $\gamma(s)$, the function $\phi(s)$ is defined as before, i.e., $\phi(s)=u(\gamma(s))$. As before we have $\phi^{\prime \prime}(s)=X_{1} X_{1} u(\gamma(s)) \geq 0$, thus concluding that $\phi$ is convex on $[-1,1]$. From this point on the proof proceeds almost verbatim as for the case of $\mathbb{H}^{1}$, and we thus leave the details to the reader.

\section{The horizontal Monge-Ampère equation.}

One of the most important aspects of the theory of convex functions is its connection with geometry through the Monge-Ampère equation. Beautiful 
accounts of this very rich and well-developed subject, including an exhaustive list of references, are given in the books $[\mathrm{Po}],[\mathrm{Ba} 3],[\mathrm{GT}],[\mathrm{CC}],[\mathrm{Gu}]$. The Monge-Ampére equation

$$
\operatorname{det} D^{2} u=f \text {, }
$$

is fully nonlinear, and becomes elliptic on $C^{2}$ strictly convex functions $u$. It is deeply connected to the geometry of the graph of $u$ in $\mathbb{R}^{n+1}$. For instance, the Gauss curvature of the graph is given by the equation

$$
K=\prod_{j=1}^{n} \kappa_{j}=\frac{\operatorname{det} D^{2} u}{\left(1+|D u|^{2}\right)^{(n+2) / 2}},
$$

where $\kappa_{1}, \ldots, \kappa_{n}$ denote the principal curvatures.

In this perspective, and in consideration of Theorem 5.12, it seems appropriate to introduce a fully nonlinear operator which becomes "elliptic" on functions which are strictly weakly $H$-convex. We expect that, similarly to its classical ancestor, such operator will play an important role in a number of problems ranging from geometry to optimal control and calculus of variations. Our intention here is simply to point at some of its basic features, deferring a systematic analysis to the forthcoming articles [DGN3], [GTo].

Definition 10.1. Let $\boldsymbol{G}$ be a Carnot group. The horizontal Monge-Ampère operator is defined on functions $u \in \Gamma^{2}(\boldsymbol{G})$ by the equation

$$
\mathcal{F}[u]=\operatorname{det}\left(X^{2} u\right)^{\star} .
$$

More in general we can consider nonlinear equations given by

$$
\mathcal{F}\left[g, u, X u,\left(X^{2} u\right)^{\star}\right]=F,
$$

where the variable $(g, u, \zeta, M)$ ranges in the set $\boldsymbol{G} \times \mathbb{R} \times \mathbb{R}^{m} \times \mathbb{R}^{m^{2}}$, with $M=\left(M_{i j}\right)$ belonging to the space of $m \times m$ symmetric matrices. The equation (10.2) is called $H$-fully nonlinear if it is not linear in the variable $M$.

Definition 10.2. The equation (10.2) is called $H$-elliptic if the matrix $\left[\frac{\partial \mathcal{F}}{\partial M_{i j}}\right]_{i, j=1, \ldots, m}$ is positive definite, i.e., if for every $\zeta \in \mathbb{R}^{m} \backslash\{0\}$ one has

$$
\sum_{i, j=1}^{m} \frac{\partial \mathcal{F}}{\partial M_{i j}} \zeta_{i} \zeta_{j}>0
$$


According to Definition 10.2, in view of Theorem 5.12 we see that the horizontal Monge-Ampère equation

$$
\operatorname{det}\left(X^{2} u\right)^{\star}=F
$$

is $H$-elliptic only for functions $u \in \Gamma^{2}(\boldsymbol{G})$ which are strictly weakly $H$ convex. In such case, the function $F$ in the right-hand side must be strictly positive.

We now return to the discussion in the closing of Section 5, with the purpose of introducing a basic definition.

Definition 10.3. Given a Carnot group $\boldsymbol{G}$ consider a function $u \in \Gamma^{2}(\boldsymbol{G})$. We define the horizontal Gaussian curvature of the graph of $u$ at the point $\left(g_{o}, u\left(g_{o}\right)\right) \in \boldsymbol{G} \times \mathbb{R}$ by the formula

$$
K_{H}\left(g_{o}\right)=\frac{\operatorname{det}\left(X^{2} u\left(g_{o}\right)\right)^{\star}}{\left(1+\left|X u\left(g_{o}\right)\right|^{2}\right)^{(m+2) / 2}} .
$$

Definition 10.3 has the following important geometric interpretation. Consider the horizontal plane $H_{g_{o}}$ passing through the point $g_{o}$, and as in (5.16) let $U_{g_{o}}$ denote the restriction of $u$ to $H_{g_{o}}$. Using the inverse of the exponential mapping we can think of $U_{g_{o}}$ as a function of the coordinates $x=x(g)=\left(x_{1}(g), \ldots, x_{m}(g)\right)$, where $\xi_{1}(g)=\sum_{\alpha=1}^{m} x_{\alpha}(g) X_{\alpha} \in V_{1}$. Using considerations similar to those that lead to (5.16), we recognize that

$$
K_{H}\left(g_{o}\right)=\frac{\operatorname{det}\left[D^{2} U_{g_{o}}\left(x\left(g_{o}\right)\right)\right]}{\left(1+\left|D U_{g_{o}}\left(x\left(g_{o}\right)\right)\right|^{2}\right)^{(m+2) / 2}} .
$$

In view of (10.1) we conclude from (10.4) that the horizontal Gaussian curvature $K_{H}\left(g_{o}\right)$ is just the classical Gaussian curvature at $\left(x\left(g_{o}\right), U_{g_{o}}\left(x\left(g_{o}\right)\right)\right.$ of the graph of the restriction of $u$ to the horizontal plane $H_{g_{o}}$. This point of view was introduced in the paper [DGN2] in connection with the theory of minimal surfaces and the Bernstein problem in Carnot groups, see also [GP], and in relation to the equation (10.3) it will be further developed in [DGN3].

We can also introduce the horizontal Gaussian curvature of an hypersurface in $\boldsymbol{G}$ defined as a level set. Consider a function $\phi \in C^{2}(\boldsymbol{G})$ and suppose that the level

$$
\Omega=\{g \in \boldsymbol{G} \mid \phi(g)<0\}
$$

is non-critical. By this we mean that there exist a neighborhood $U$ of $\mathcal{S}=$ $\partial \Omega$, and $\delta>0$, such that the Riemaniann gradient of $\phi$ satisfy

$$
|D \phi(g)| \geq \delta, \quad \text { for every } \quad g \in U .
$$


In this case $S$ constitutes a $C^{2}$ hypersurface in $\boldsymbol{G}$. We assume that the point $g_{o} \in S$ be non-characteristic, i.e., that we have $\left|X \phi\left(g_{o}\right)\right| \neq 0$. By continuity, this inequality continues to be valid in a full neighborhood of $g_{o}$. We consider the restriction $\Phi_{g_{o}}$ of the defining function $\phi$ to the horizontal plane $H_{g_{o}}$, so that as above we can think of $\Phi_{g_{o}}$ as a function of the variable $x=x(g) \in \mathbb{R}^{m}$. Let

$$
S_{g_{o}}=\left\{x \in \mathbb{R}^{m} \mid \Phi_{g_{o}}(x)<0\right\},
$$

and notice that, thanks to the non-characteristic assumption, and to (5.16), the set $S_{g_{o}}$ is a $C^{2}$ hypersurface in $\mathbb{R}^{m}$ nearby $x\left(g_{o}\right) \cong \xi_{1}\left(g_{o}\right)=$ $\sum_{\alpha=1}^{m} x_{\alpha}\left(g_{o}\right) X_{\alpha}$. Set $x_{o}=x\left(g_{o}\right) \in \mathbb{R}^{m}$, and denote by $\kappa_{i}\left(x_{o}\right), i=1, \ldots, m-1$ the principal curvatures of $S_{g_{o}}$ at $x_{o}$. We define the horizontal Gaussian curvature of $S$ at $g_{o}$ as the standard Gaussian curvature of $S_{g_{o}}$ at $x_{o}$, i.e.,

$$
K_{H}\left(S ; g_{o}\right) \stackrel{\text { def }}{=} \prod_{i=1}^{m-1} \kappa_{i}\left(x_{o}\right)
$$

When the point $g_{o}$ is a characteristic point of the hypersurface $S$, then equation (5.16) gives

$$
D \Phi_{g_{o}}\left(x_{o}\right)=X \phi\left(g_{o}\right)=0
$$

and we cannot apply the above considerations. In this case the situation is more complex, as one needs to identify geometric conditions on $S$ near $g_{o}$ which allow to define the horizontal principal curvatures by appropriate limiting relations. For these aspects we refer the reader to the forthcoming article [DGN3].

We close this section by introducing some geometric notions of convexity which will prove important in the study of the equation (10.3), and of its connections to the problem stated in the introduction. In the process, we will also propose an appropriate notion of viscosity solution. We begin by developing a few preliminary properties. We recall Definition 3.6 of an $\mathrm{H}$ affine function $A: G \rightarrow \mathbb{R}$

$$
A\left(g^{\prime}\right)=<p, \xi_{1}\left(g^{\prime}\right)>+\beta \quad g^{\prime} \in \boldsymbol{G} .
$$

Given an open set $\Omega \subset \boldsymbol{G}$, and a function $u: \Omega \rightarrow \mathbb{R}$, we say that an $H$-affine function $A$ supports $u$ at $g_{o} \in \Omega$ if

$$
A\left(g_{o}\right)=u\left(g_{o}\right), \quad \text { and } \quad A\left(g^{\prime}\right) \leq u\left(g^{\prime}\right) \quad \text { for every } \quad g^{\prime} \in \Omega \cap H_{g_{o}} .
$$


Clearly, the first condition in (10.5) implies the existence of $p\left(g_{o}\right) \in V_{1}$ such that

$$
A\left(g^{\prime}\right)=u\left(g_{o}\right)+<p\left(g_{o}\right), \xi_{1}\left(g^{\prime}\right)-\xi_{1}\left(g_{o}\right)>,
$$

for all $g^{\prime} \in \boldsymbol{G}$. The vector $p\left(g_{o}\right)$ is called a horizontal subgradient of $u$ at $g_{o}$.

Definition 10.4. We define the horizontal normal mapping of $u$ as the setvalued function $\partial_{H} u: \Omega \rightarrow \mathcal{P}\left(V_{1}\right)$ given by

$$
\partial_{H} u\left(g_{o}\right)=\left\{p\left(g_{o}\right) \mid p\left(g_{o}\right) \text { is a horizontal subgradient for } u \text { at } g_{o}\right\} \text {. }
$$

For any $E \subset \Omega$ we set

$$
\partial_{H} u(E)=\bigcup_{g_{o} \in E} \partial_{H} u\left(g_{o}\right)
$$

The horizontal lower contact set of $u$ in $\Omega$ is defined as follows

$$
\Gamma_{H}^{-}(u ; \Omega)=\left\{g_{o} \in \Omega \mid \partial_{H} u\left(g_{o}\right) \neq \emptyset\right\} .
$$

It is clear from (10.5) and (10.6) that $\partial_{H} u\left(g_{o}\right) \neq \emptyset$ if and only if $u$ admits a support at $g_{o}$.

Proposition 10.5. Let $\Omega \subset G$ be an open weakly $H$-convex set, if

$$
\Gamma_{H}^{-}(u ; \Omega)=\Omega
$$

then $u \in \mathcal{C}_{H}^{w}(\Omega)$.

Proof. Fix arbitrarily $g_{o} \in \Omega$, we need to show that with $g_{\lambda}=g_{o} \delta_{\lambda}\left(g_{o}^{-1} g\right)$

$$
u\left(g_{\lambda}\right) \leq(1-\lambda) u\left(g_{o}\right)+\lambda u(g)
$$

for any $g \in \Omega \cap H_{g_{o}}$, and every $\lambda \in[0,1]$. From Proposition 4.2 we know $g_{\lambda} \in \Omega \cap H_{g_{o}}$. Let $A$ be a support for $u$ at $g_{\lambda}$. From (10.6) we have

$$
A\left(g^{\prime}\right)=u\left(g_{\lambda}\right)+<p\left(g_{\lambda}\right), \xi_{1}\left(g^{\prime}\right)-\xi_{1}\left(g_{\lambda}\right)>\quad g^{\prime} \in \Omega,
$$

for some $p\left(g_{\lambda}\right) \in V_{1}$, whereas (10.5) gives

$$
u\left(g_{\lambda}\right)+<p\left(g_{\lambda}\right), \xi_{1}\left(g^{\prime}\right)-\xi_{1}\left(g_{\lambda}\right)>\leq u\left(g^{\prime}\right) \quad \text { for every } \quad g^{\prime} \in \Omega \cap H_{g_{\lambda}} .
$$


It is now easy to verify that

$$
u\left(g_{\lambda}\right)=(1-\lambda) A\left(g_{o}\right)+\lambda A(g) .
$$

We have in fact from $(2.7)$

$$
\xi_{1}\left(g_{\lambda}\right)=\xi_{1}\left(g_{o}\right)+\lambda\left[\xi_{1}(g)-\xi_{1}\left(g_{o}\right)\right],
$$

and therefore (10.7) gives

$$
\begin{aligned}
(1-\lambda) A\left(g_{o}\right)+\lambda A(g) & =(1-\lambda)\left\{u\left(g_{\lambda}\right)+<p\left(g_{\lambda}\right), \xi_{1}\left(g_{o}\right)-\xi_{1}\left(g_{\lambda}\right)>\right\} \\
& +\lambda\left\{u\left(g_{\lambda}\right)+<p\left(g_{\lambda}\right), \xi_{1}(g)-\xi_{1}\left(g_{\lambda}\right)>\right\} \\
& =u\left(g_{\lambda}\right)
\end{aligned}
$$

which proves (10.9). Using (10.9), we would be finished if we could say that

$$
A\left(g_{o}\right) \leq u\left(g_{o}\right), \quad A(g) \leq u(g) .
$$

These inequalities would follow from (10.8) if we knew that

$$
g_{o} \in H_{g_{\lambda}}, \quad g \in H_{g_{\lambda}} .
$$

The former relation is obvious from Proposition 4.2 since $g \in H_{g_{o}}$ implies $g_{\lambda} \in H_{g_{o}}$, and this is equivalent to $g_{o} \in H_{g_{\lambda}}$. The second relation is less obvious, but it is equally true. It suffices to observe that $g \in H_{g_{\lambda}}$ is equivalent to $g_{\lambda}^{-1} g \in H_{e}$, which is the same as $g^{-1} g_{\lambda} \in H_{e}$. Now we can write this as $g^{-1} g_{\lambda}=h^{-1} \delta_{\lambda} h \in H_{e}$, where $h=g_{o}^{-1} g$. To see that this latter relation is true it is enough to observe that $g \in H_{g_{o}}$ is equivalent to $h \in H_{e}$, and then use the Baker-Campbell-Hausdorff formula (2.7) to verify that for any $h \in H_{e}$ one has $h^{-1} \delta_{\lambda} h \in H_{e}$. This completes the proof of the proposition.

Proposition 10.6. Let $\Omega \subset \boldsymbol{G}$ be an open set, and let $u \in \Gamma^{1}(\Omega)$. If $g_{o} \in \Gamma_{H}^{-}(u ; \Omega)$, then

$$
\partial_{H} u\left(g_{o}\right)=\left\{X u\left(g_{o}\right)\right\} .
$$

If furthermore $u \in \Gamma^{2}(\Omega)$, then

$$
\left(X^{2} u\left(g_{o}\right)\right)^{\star} \geq 0 .
$$

Proof. By the assumption $g_{o} \in \Gamma_{H}^{-}(u ; \Omega)$ we know that $\partial_{H} u\left(g_{o}\right) \neq \emptyset$. To establish the first part we show that if $p\left(g_{o}\right) \in \partial_{H} u\left(g_{o}\right)$, then $p=X u\left(g_{o}\right)$. Now if $p\left(g_{o}\right) \in \partial_{H} u\left(g_{o}\right)$ we have

$$
u\left(g_{o}\right)+<p\left(g_{o}\right), \xi_{1}\left(g^{\prime}\right)-\xi_{1}\left(g_{o}\right)>\leq u\left(g^{\prime}\right), \quad g^{\prime} \in \Omega \cap H_{g_{o}} .
$$


Fix $g \in \Omega \cap H_{g_{o}}$, and consider $g_{\lambda}=g_{o} \delta_{\lambda}\left(g_{o}^{-1} g\right)$. By Proposition 4.2 we know $g_{\lambda} \in \Omega \cap H_{g_{o}}$, therefore the previous inequality applied to $g^{\prime}=g_{\lambda}$ gives

$$
<p\left(g_{o}\right), \xi_{1}\left(g_{\lambda}\right)-\xi_{1}\left(g_{o}\right)>\leq u\left(g_{\lambda}\right)-u\left(g_{o}\right),
$$

which, using (2.7), we can rewrite

$$
<p\left(g_{o}\right), \xi_{1}(g)-\xi_{1}\left(g_{o}\right)>\leq \frac{u\left(g_{\lambda}\right)-u\left(g_{o}\right)}{\lambda} .
$$

Passing to the limit as $\lambda \rightarrow 0$ in the latter inequality, invoking Proposition 2.3 we conclude

$$
<p\left(g_{o}\right), \xi_{1}(g)-\xi_{1}\left(g_{o}\right)>\leq<X u\left(g_{o}\right), \xi_{1}(g)-\xi_{1}\left(g_{o}\right)>\quad g \in \Omega \cap H_{g_{o}} .
$$

By left-translation this inequality can be equivalently rewritten as follows

$$
<p\left(g_{o}\right), \xi_{1}(h)>\leq<X u\left(g_{o}\right), \xi_{1}(h)>\quad h \in \tilde{\Omega} \cap H_{e},
$$

where we have let $\tilde{\Omega}=g_{o}^{-1} \Omega$. Since the open set $\tilde{\Omega}$ contains the identity, there exists $R>0$ sufficiently small such that $\bar{B}(e, R) \subset \tilde{\Omega}$, therefore the latter inequality holds in particular for every $h \in \bar{B}(e, R) \cap H_{e}$. However, $\bar{B}(e, R) \cap H_{e}$ is closed under the inversion $h \rightarrow h^{-1}$, and on the other hand $\xi_{1}\left(h^{-1}\right)=-\xi_{1}(h)$. Since when $h \in \bar{B}(e, R) \cap H_{e}$ the vector $\xi_{1}(h)$ describes the set $B_{e}(0, R)$, the $m$-dimensional Euclidean ball in $V_{1}$ centered at the origin and having radius $R$, we thus conclude that it must be $p\left(g_{o}\right)=X u\left(g_{o}\right)$. This completes the proof of the first part. The second part now follows from the first, and from the proof of the necessity in Theorem 5.12, see also Theorem 5.11 .

We next compute an important example of normal mapping.

Proposition 10.7. Let $\boldsymbol{G}$ be a Carnot group and consider the gauge pseudo-ball $B\left(g_{o}, R\right)$. For any $a>0$ let

$$
u(g) \stackrel{\text { def }}{=} \frac{a}{R} d\left(g, g_{o}\right) .
$$

The graph of $u$ is the cone in $\boldsymbol{G} \times \mathbb{R}$ with vertex at $\left(g_{o}, 0\right)$ and basis $B\left(g_{o}, R\right) \times$ $\{a\}$. The horizontal normal mapping of $u$ is given by

$$
\partial_{H} u(g)= \begin{cases}\frac{a}{R} X d\left(g, g_{o}\right) & g \neq g_{o}, \\ \bar{B}_{e}\left(0, \frac{a}{R}\right) & g \neq g_{o},\end{cases}
$$


where we have denoted by $B_{e}\left(0, \frac{a}{R}\right)$ the $m$-dimensional Euclidean ball in $V_{1}$ centered at the origin and having radius $a / R$.

Proof. From the definition (2.12) of the pseudo-distance we see that $g \rightarrow$ $d\left(g, g_{o}\right)$ belongs to $C^{\infty}\left(\boldsymbol{G} \backslash\left\{g_{o}\right\}\right)$, therefore $\partial_{H} u(g)=\frac{a}{R} X d\left(g, g_{o}\right)$ for $g \neq g_{o}$ immediately follows from Proposition 10.6. Next, we observe that $p \in V_{1}$ belongs to $\partial_{H} u\left(g_{o}\right)$ if and only if

$$
<p, \xi_{1}(g)-\xi_{1}\left(g_{o}\right)>\leq \frac{a}{R} d\left(g, g_{o}\right)
$$

for every $g \in B\left(g_{o}, R\right) \cap H_{g_{o}}$. By invariance with respect to left-translation the latter inequality is equivalent to

$$
<p, \xi_{1}(h)>\leq \frac{a}{R} N(h)
$$

for every $h \in B(e, R) \cap H_{e}$. In view of (9.4) we conclude that $p \in \partial_{H} u\left(g_{o}\right)$ if and only if

$$
<p, \frac{\xi_{1}(h)}{\left|\xi_{1}(h)\right|}>\leq \frac{a}{R}
$$

for every $h \in B(e, R) \cap H_{e}$. This is like saying that $p \in \bar{B}_{e}(0, a / R)$.

The converse to Proposition 10.5 is a rather delicate point which we plan to discuss in [DGN3]. Here, to continue our discussion, we a priori assume such property. That is we consider, for a fixed weakly $H$-convex open set $\Omega \subset \boldsymbol{G}$, a function $u \in \mathcal{C}_{H}^{w}(\Omega) \cap C(\Omega)$, such that

$$
\partial_{H} u\left(g_{o}\right) \neq \emptyset \quad \text { for every } \quad g_{o} \in \Omega \text {. }
$$

We stress explicitly that when $u \in C_{H}^{w}(\Omega) \cap \Gamma^{1}(\Omega)$, then thanks to Proposition 10.6 we have $\partial_{H} u\left(g_{o}\right)=\left\{X u\left(g_{o}\right)\right\}$, and therefore (10.10) is guaranteed.

Suppose now that $\phi \in \Gamma^{2}(\Omega)$ and that $u-\phi$ restricted to the horizontal plane $H_{g_{o}}$ has a local maximum in $g_{o} \in \Omega$. We claim that it must be

$$
\operatorname{det}\left(X^{2} \phi\left(g_{o}\right)\right)^{\star} \geq 0
$$

To prove (10.11) we argue as follows. We fix arbitrarily $g \in \Omega \cap H_{g_{o}}$ and consider the function $\psi(\lambda)=\phi\left(g_{\lambda}\right)$, where $g_{\lambda}=g_{o} \delta_{\lambda}\left(g_{o}^{-1} g\right)$. ¿From the assumption on $\phi$ we have $\psi \in C^{2}(0,1)$, therefore Taylor's formula gives

$$
\psi(\lambda)=\psi(0)+\psi^{\prime}(0) \lambda+\frac{\psi^{\prime \prime}(0)}{2} \lambda^{2}+o\left(\lambda^{2}\right) .
$$


From the latter equation, and from (5.12) and (5.13) in Proposition 5.4, we conclude

$$
\begin{aligned}
\phi\left(g_{\lambda}\right) & =\phi\left(g_{o}\right)+<X \phi\left(g_{o}\right), \xi_{1}(g)-\xi_{1}\left(g_{o}\right)>\lambda \\
& +\frac{1}{2}<\left(X^{2} \phi\left(g_{o}\right)\right)^{\star}\left(\xi_{1}(g)-\xi_{1}\left(g_{o}\right)\right),\left(\xi_{1}(g)-\xi_{1}\left(g_{o}\right)\right)>\lambda^{2}+o\left(\lambda^{2}\right) .
\end{aligned}
$$

From Proposition 4.2 and from the weak $H$-convexity of $\Omega$ we know that $g_{\lambda} \in \Omega \cap H_{g_{o}}$ for every $\lambda \in[0,1]$. Since the restriction of $u-\phi$ to $H_{g_{o}}$ has a local maximum in $g_{o}$, we conclude that there exists $\lambda_{o}>0$ sufficiently small such that

$$
u\left(g_{\lambda}\right) \leq \phi\left(g_{\lambda}\right)+u\left(g_{o}\right)-\phi\left(g_{o}\right) \quad \text { for } 0<\lambda<\lambda_{o} .
$$

Inserting (10.12) in the latter inequality we find

$$
\begin{aligned}
u\left(g_{\lambda}\right) & \leq u\left(g_{o}\right)+<X \phi\left(g_{o}\right), \xi_{1}(g)-\xi_{1}\left(g_{o}\right)>\lambda \\
& +\frac{1}{2}<\left(X^{2} \phi\left(g_{o}\right)\right)^{\star}\left(\xi_{1}(g)-\xi_{1}\left(g_{o}\right)\right),\left(\xi_{1}(g)-\xi_{1}\left(g_{o}\right)\right)>\lambda^{2}+o\left(\lambda^{2}\right) .
\end{aligned}
$$

From the assumption (10.10) we infer the existence of $p\left(g_{o}\right) \in V_{1}$ such that

$$
<p\left(g_{o}\right), \xi_{1}\left(g^{\prime}\right)-\xi_{1}\left(g_{o}\right)>\leq u\left(g^{\prime}\right)-u\left(g_{o}\right) \quad \text { for every } \quad g^{\prime} \in \Omega \cap H_{g_{o}} \text {. }
$$

Taking $g^{\prime}=g_{\lambda}$ in the latter inequality, and substituting in (10.13) we find

$$
\begin{aligned}
& <p\left(g_{o}\right), \xi_{1}\left(g_{\lambda}\right)-\xi_{1}\left(g_{o}\right)>\leq<X \phi\left(g_{o}\right), \xi_{1}(g)-\xi_{1}\left(g_{o}\right)>\lambda \\
& +\frac{1}{2}<\left(X^{2} \phi\left(g_{o}\right)\right)^{\star}\left(\xi_{1}(g)-\xi_{1}\left(g_{o}\right)\right),\left(\xi_{1}(g)-\xi_{1}\left(g_{o}\right)\right)>\lambda^{2}+o\left(\lambda^{2}\right) .
\end{aligned}
$$

By (2.7) one has $\xi_{1}\left(g_{\lambda}\right)-\xi_{1}\left(g_{o}\right)=\lambda\left(\xi_{1}(g)-\xi_{1}\left(g_{o}\right)\right)$, therefore we obtain from (10.14)

$$
\begin{aligned}
(10.15) & <p\left(g_{o}\right), \xi_{1}\left(g_{1}\right)-\xi_{1}\left(g_{o}\right)>\leq<X \phi\left(g_{o}\right), \xi_{1}(g)-\xi_{1}\left(g_{o}\right)> \\
& +\frac{1}{2}<\left(X^{2} \phi\left(g_{o}\right)\right)^{\star}\left(\xi_{1}(g)-\xi_{1}\left(g_{o}\right)\right),\left(\xi_{1}(g)-\xi_{1}\left(g_{o}\right)\right)>\lambda+o(\lambda) .
\end{aligned}
$$


Letting $\lambda \rightarrow 0$ in (10.15) gives

$$
<p\left(g_{o}\right), \xi_{1}(g)-\xi_{1}\left(g_{o}\right)>\leq<X \phi\left(g_{o}\right), \xi_{1}(g)-\xi_{1}\left(g_{o}\right)>.
$$

By the arbitrariness of $g \in \Omega \cap H_{g_{o}}$ we conclude from (10.16) that must be $p\left(g_{o}\right)=X \phi\left(g_{o}\right)$. But then (10.15) implies

$$
<\left(X^{2} \phi\left(g_{o}\right)\right)^{\star}\left(\xi_{1}(g)-\xi_{1}\left(g_{o}\right)\right),\left(\xi_{1}(g)-\xi_{1}\left(g_{o}\right)\right)>\geq 0 .
$$

Again the arbitrariness of $g$ shows that the matrix $\left(X^{2} \phi\left(g_{o}\right)\right)^{\star}$ is semidefinite positive, and therefore (10.11) holds. This proves the claim.

These considerations, and the results of Sections 5 and 7, suggest an appropriate notion of viscosity solution for the equation 10.3 which will prove central in its study. Although such notion is inspired to the by now classical one due to Crandall-Ishii-Lions [CIL], it is essentially modelled on that of weakly $H$-convex function, and therefore one has to necessarily confront the delicate problems deriving from an information on lower dimensional manifolds.

Definition 10.8. Given a weakly $H$-convex open set $\Omega$ in a Carnot group $\boldsymbol{G}$, and a function $F \in C(\Omega), F \geq 0$, we say that a function $u \in C(\Omega) \cap \mathcal{C}_{H}^{w}(\Omega)$ satisfying (10.10) is a viscosity subsolution (supersolution) to (10.3) if for any $\phi \in \Gamma^{2}(\Omega) \cap \mathcal{C}_{H}^{w}(\Omega)$ and $g_{o} \in \Omega$ such that the restriction of $u-\phi$ to the horizontal plane $H_{g_{o}}$ has a local maximum (minimum) at $g_{o}$, then it must be

$$
\operatorname{det}\left[\left(X^{2} \phi\left(g_{o}\right)\right)^{\star}\right] \geq(\leq) F\left(g_{o}\right) \text {. }
$$

If $u$ is at the same time a viscosity sub/supersolution, then we say that it is a viscosity solution to (10.3).

The notion of viscosity solution is intimately connected to that of solution in the sense of the horizontal Monge-Ampère measure associated with the horizontal normal mapping $\partial_{H} u$. The latter is defined by the equation

$$
M_{H} u(E) \stackrel{\text { def }}{=} \mathcal{H}^{m}\left(\partial_{H} u(E)\right),
$$

where $\mathcal{H}^{m}$ denotes the standard $m$-dimensional Hausdorff measure on the Lie algebra $\mathfrak{g}$ (we recall that $m=\operatorname{dim} V_{1}$ ), and $E \subset \Omega$ is such that $\partial_{H} u(E)$ is $\mathcal{H}^{m}$-measurable. We will come back in [DGN3] to the fundamental question of the equivalence of these two notions of solutions.

We close this section with one more witness of the remarkable role played by the gauge in groups of Heisenberg type. Combined with Theorem 8.1, the next result states that the gauge is a fundamental solution of the horizontal Monge-Ampère equation. 
Theorem 10.9. Let $G$ be a group of Heisenberg type and $N(g)$ be the gauge defined in (6.3), then

$$
\operatorname{det}\left(X^{2} N(g)\right)^{\star}=0 \quad \text { if } g \neq e .
$$

Proof. To prove the theorem we show that the horizontal Hessian matrix $\left(X^{2} N(g)\right)^{\star}$ is singular for $g \neq e$ by exhibiting a non-zero vector $p=p(g) \in$ $\mathbb{R}^{m} \simeq V_{1}$ in the kernel of the corresponding linear map associated to this matrix. In fact, we take $p=X u=\left(X_{1} u, \ldots, X_{m} u\right)$ where $u(g)=N(g)^{4}$. Since by (6.23),

$$
\left(X^{2} N\right)^{\star}=\frac{1}{4 N^{3}}\left[\left(X^{2} u\right)^{\star}-\frac{3}{4 N^{4}}(X u \otimes X u)\right],
$$

to prove the theorem it will suffice to show

$$
\left[\left(X^{2} u\right)^{\star}-\frac{3}{4 N^{4}}(X u \otimes X u)\right](X u)=0 \quad \text { in } \quad \boldsymbol{G} \backslash\{e\} .
$$

To this end, we first observe that $X u=4 N^{3} X N$, hence using (6.11) in Lemma 6.3 we find

$$
|X u|^{2}=16 N^{6}|X N|^{2}=16 N^{4} \psi .
$$

On the other hand, we have $X u=X \psi^{2}+16 X \chi$, and so we find from $(6.20),(6.24)$

$$
X u=4 \psi \xi_{1}+16 J\left(\xi_{2}\right) \xi_{1} .
$$

Since $(X u \otimes X u) X u=|X u|^{2} X u$, we conclude

$$
\frac{3}{4 N^{4}}[X u \otimes X u](X u)=12 \psi X u=12 \psi\left\{4 \psi \xi_{1}+16 J\left(\xi_{2}\right) \xi_{1}\right\} .
$$

We next turn to the computation of $\left(X^{2} u\right)^{\star}(X u)$. By (6.19) we have

$$
\begin{aligned}
\left(X^{2} u\right)^{\star}(X u) & =\left(X^{2} \psi^{2}\right)^{\star}(X u)+16\left(X^{2} \chi\right)^{\star}(X u) \\
& =\left(X^{2} \psi^{2}\right)^{\star}\left(X \psi^{2}\right)+16\left(X^{2} \psi^{2}\right)^{\star}(X \chi) \\
& +16\left(X^{2} \chi\right)^{\star}\left(X \psi^{2}\right)+16^{2}\left(X^{2} \chi\right)^{\star}(X \chi) .
\end{aligned}
$$

Using (6.21) one easily recognizes that

$$
\left(X^{2} \psi^{2}\right)^{\star}\left(X \psi^{2}\right)=48 \psi^{2} \xi_{1} .
$$


¿From (6.21) and from (6.24) we obtain for the $\alpha$-th component of $16\left(X^{2} \psi^{2}\right)^{\star}(X \chi)$

$$
\begin{aligned}
& 16 \sum_{\alpha^{\prime}=1}^{m}\left(4 \delta_{\alpha \alpha^{\prime}} \psi+8 x_{\alpha} x_{\alpha^{\prime}}\right)<J\left(\xi_{2}\right) \xi_{1}, X_{\alpha^{\prime}}> \\
& =16\left(4 \psi<J\left(\xi_{2}\right) \xi_{1}, X_{\alpha}>+8 x_{\alpha}<J\left(\xi_{2}\right) \xi_{1}, \xi_{1}>\right) .
\end{aligned}
$$

From this formula we conclude

$$
16\left(X^{2} \psi^{2}\right)^{\star}(X \chi)=64 \psi J\left(\xi_{2}\right) \xi_{1} .
$$

Next, from (6.16) and (6.20) we obtain for the $\alpha$-th component of $16\left(X^{2} \chi\right)^{\star}\left(X \psi^{2}\right)$

$$
\begin{aligned}
& 32 \psi \sum_{\beta=1}^{k} \sum_{\alpha^{\prime}=1}^{m}<\left[\xi_{1}, X_{\alpha}\right], Y_{\beta}><\left[\xi_{1}, X_{\alpha^{\prime}}\right], Y_{\beta}>x_{\alpha^{\prime}} \\
& =32 \psi \sum_{\beta=1}^{k}<\left[\xi_{1}, X_{\alpha}\right], Y_{\beta}><J\left(Y_{\beta}\right) \xi_{1}, \xi_{1}>=0 .
\end{aligned}
$$

This gives

$$
16\left(X^{2} \chi\right)^{\star}\left(X \psi^{2}\right)=0 .
$$

Finally, from (6.16) and (6.24) we find for the $\alpha$-th component of $16^{2}\left(X^{2} \chi\right)^{\star}(X \chi)$

$$
\begin{aligned}
& \frac{16^{2}}{2} \sum_{\beta=1}^{k}<\left[\xi_{1}, X_{\alpha}\right], Y_{\beta}>\sum_{\alpha^{\prime}=1}^{m}<\left[\xi_{1}, X_{\alpha^{\prime}}\right], Y_{\beta}><J\left(\xi_{2}\right) \xi_{1}, X_{\alpha^{\prime}}> \\
& =\frac{16^{2}}{2} \sum_{\beta=1}^{k}<\left[\xi_{1}, X_{\alpha}\right], Y_{\beta}><J\left(Y_{\beta}\right) \xi_{1}, J\left(\xi_{2}\right) \xi_{1}> \\
& =\frac{16^{2}}{2} \sum_{\beta=1}^{k}<\left[\xi_{1}, X_{\alpha}\right], Y_{\beta}><Y_{\beta}, \xi_{2}>\left|\xi_{1}\right|^{2} \\
& =\frac{16^{2}}{2} \psi<\left[\xi_{1}, X_{\alpha}\right], \xi_{2}>=\frac{16^{2}}{2} \psi<J\left(\xi_{2}\right) \xi_{1}, X_{\alpha}>.
\end{aligned}
$$

This gives

$$
16^{2}\left(X^{2} \chi\right)^{\star}(X \chi)=\frac{16^{2}}{2} \psi J\left(\xi_{2}\right) \xi_{1}
$$


Substituting (10.21) - (10.24) in (10.20), we obtain

$$
\left(X^{2} u\right)^{\star}(X u)=12 \psi\left\{4 \psi \xi_{1}+16 J\left(\xi_{2}\right) \xi_{1}\right\} .
$$

Comparing (10.25) with (10.19) we conclude that (10.17) holds. This completes the proof.

\section{Yet another notion of convexity.}

The most elementary properties of classical convex functions are their being locally bounded and continuous on their effective domain. Corresponding properties for weakly $H$-convex functions are far from obvious and are presently being investigated. The purpose of this section is to prove that by strengthening Definition 5.5, but of course without falling back into the notion of strong $H$-convexity, one obtains an "intermediate" class of convex functions which, remarkably, share with the classical ones the above mentioned properties. This implies in particular that the results in Sections 8 and 9 are valid generically for such functions, without any additional assumption.

To set the stage, consider in the Heisenberg group a function $u: \mathbb{H}^{n} \rightarrow \mathbb{R}$. For every $g_{o}=\left(x_{o}, y_{o}, t_{o}\right) \in \mathbb{H}^{n}$ denote by $U_{g_{o}}$ the restriction of $u$ to the horizontal plane $H_{g_{o}}$, i.e.,

$U_{g_{o}}(x, y) \stackrel{\text { def }}{=} u\left(x, y, t_{o}+2\left(<y_{o}, x>-<x_{o}, y>\right)\right), \quad(x, y) \in \mathbb{R}^{2 n}$.

Definition 11.1. A function $u: \mathbb{H}^{n} \rightarrow \mathbb{R}$ is called $H$-convex if for any fixed $g_{o} \in \mathbb{H}^{n}$ the function $U_{g_{o}}$ is an ordinary convex function of the variables $(x, y) \in \mathbb{R}^{2 n}$. The class of all $H$-convex functions will be denoted by $C_{H}\left(\mathbb{H}^{n}\right)$.

To understand better Definition 11.1, consider $u \in C_{H}\left(\mathbb{H}^{n}\right)$. For any $g_{o} \in \mathbb{H}^{n},(x, y),\left(x^{\prime}, y^{\prime}\right) \in \mathbb{R}^{2 n}$, and $0 \leq \lambda \leq 1$ one has

$$
U_{g_{o}}\left(x+\lambda\left(x^{\prime}-x\right), y+\lambda\left(y^{\prime}-y\right)\right) \leq(1-\lambda) U_{g_{o}}(x, y)+\lambda U_{g_{o}}\left(x^{\prime}, y^{\prime}\right) .
$$

Equivalently, we can write

$$
\begin{aligned}
& u\left(x+\lambda\left(x^{\prime}-x\right), y+\lambda\left(y^{\prime}-y\right),\right. \\
& \left.\quad t_{o}+2\left[<y_{o}, x+\lambda\left(x^{\prime}-x\right)>-<x_{o}, y+\lambda\left(y^{\prime}-y\right)>\right]\right) \\
& \leq(1-\lambda) u\left(x, y, t_{o}+2\left(<y_{o}, x>-<x_{o}, y>\right)\right) \\
& +\lambda u\left(x^{\prime}, y^{\prime}, t_{o}+2\left(<y_{o}, x^{\prime}>-<x_{o}, y^{\prime}>\right)\right) .
\end{aligned}
$$


If we now define $t=t_{o}+2\left(<y_{o}, x>-<x_{o}, y>\right), t^{\prime}=t_{o}+2\left(<y_{o}, x^{\prime}>\right.$ $\left.-<x_{o}, y^{\prime}>\right)$, then it is clear that the points $g=(x, y, t), g^{\prime}=\left(x^{\prime}, y^{\prime}, t^{\prime}\right)$ belong to $H_{g_{o}}$, and since $\left.t-t=2\left(<y_{o}, x^{\prime}-x>-<x_{o}, y^{\prime}-y\right\rangle\right)$, the latter inequality is equivalent to writing

$u\left((1-\lambda) g+\lambda g^{\prime}\right) \leq(1-\lambda) u(g)+\lambda u\left(g^{\prime}\right), \quad g, g^{\prime} \in H_{g_{o}}, 0 \leq \lambda \leq 1$,

where we have denoted with $(1-\lambda) g+\lambda g^{\prime}$ the ordinary Euclidean convex combination of $g, g^{\prime}$, as points of $\mathbb{R}^{2 n+1}$. We conclude that $u$ is $H$-convex if and only if given any horizontal plane $H_{g_{o}} \subset \mathbb{H}^{n}$, the inequality (11.1) holds.

We remark explicitly that Definition 11.1 continues to make sense in any Carnot group. It is also immediate to recognize that if a function $u$ is (Euclidean) convex in the ordinary sense at the level of the Lie algebra, then it is also $H$-convex. In fact, a function $f: \mathbb{R}^{N} \rightarrow \mathbb{R}$ is convex if and only if its restriction to any hyperplane in $\mathbb{R}^{N}$ is convex. Since the notion of $H$-convexity involves restricting only to horizontal planes, it is clear that the class $C_{H}\left(\mathbb{H}^{n}\right)$ includes Euclidean convex functions. A comparison with our Definition 5.5 reveals that any function which is $H$-convex is in particular weakly $H$-convex since we only request convexity with respect to the base point $g_{o}$. The next result shows that the inclusion between the two classes is strict.

Proposition 11.2. If $u: \mathbb{H}^{n} \rightarrow \mathbb{R}$ is $H$-convex, then it is also weakly $H$ convex. Furthermore, there exists weakly $H$-convex functions which are not $H$-convex. The gauge $N(g)$ is an example of such a function.

Proof. The inclusion $C_{H}\left(\mathbb{H}^{n}\right) \subset \mathcal{C}_{H}^{w}\left(\mathbb{H}^{n}\right)$ is clear since for any given $g \in \mathbb{H}^{n}$, Definition 11.1 gives for any $g^{\prime} \in H_{g}$ and $0 \leq \lambda \leq 1$

$$
u\left((1-\lambda) g+\lambda g^{\prime}\right) \leq(1-\lambda) u(g)+\lambda u\left(g^{\prime}\right) .
$$

On the other hand, Proposition 4.4 implies

$$
(1-\lambda) g+\lambda g^{\prime}=g_{\lambda}
$$

which shows that if $u \in C_{H}$, then also $u \in \mathcal{C}_{H}^{w}$.

To prove that the inclusion is strict, consider in the Heisenberg group $\mathbb{H}^{1}$ the gauge $N(g)=\left(\left(x^{2}+y^{2}\right)^{2}+t^{2}\right)^{1 / 4}$. According to Theorem $6.8, N(g)$ is weakly $H$-convex. However, it is not $H$-convex. To see this consider the 
point $g_{o}=(-1 / 2,0,0)$. The horizontal plane $H_{g_{o}}$ has equation $t=y$, and therefore the restriction of $N(g)$ to $H_{g_{o}}$ is given by

$$
U_{g_{o}}(x, y)=\left(\left(x^{2}+y^{2}\right)^{2}+y^{2}\right)^{1 / 4}, \quad(x, y) \in \mathbb{R}^{2} .
$$

If $U_{g_{o}}(x, y)$ were an ordinary convex function, then its restriction to the $y$-axis

$$
U_{g_{o}}(0, y)=\left(y^{4}+y^{2}\right)^{1 / 4}
$$

would be a convex function of $y$, and this is clearly false since $U_{g_{o}}(0, y) \approx$ $|y|^{1 / 2}$ for $|y| \approx 0$.

A basic property of classical convex functions is that they are locally bounded in the interior of their effective domain, and furthermore continuous. Such properties are far from obvious in the non abelian setting of this paper. The purpose of what follows is to prove that $H$-convex functions enjoy them generically. Our first theorem in this direction proves the local boundedness of $u$. In preparation for it, we recall the following classical result, see Theorem 1.5.1 in [Sch].

Theorem 11.3. Let $f: \mathbb{R}^{N} \rightarrow \mathbb{R}$ be a convex function. For any ball $B_{e}\left(x_{o}, R\right)$, consider a (closed) simplex $S$ formed by the points $\left\{x_{1}, \ldots, x_{N+1}\right\}$, and such that $\bar{B}_{e}\left(x_{o}, R\right) \subset \stackrel{\circ}{S}$. Let $\mathcal{M}=\max \left\{f\left(x_{i}\right) \mid i=1, \ldots, N+1\right\}$, then one has

$$
\left|f(y)-f\left(x_{o}\right)\right| \leq\left[\mathcal{M}-f\left(x_{o}\right)\right] \frac{\left|y-x_{o}\right|}{R}, \quad \text { for every } y \in \bar{B}_{e}\left(x_{o}, R\right) .
$$

Furthermore,

$|f(y)-f(x)| \leq 4\left\{\left[\mathcal{M}-f\left(x_{o}\right)\right]+\left|f\left(x_{o}\right)\right|\right\} \frac{|y-x|}{R} \quad x, y \in \bar{B}_{e}\left(x_{o}, R / 2\right)$.

In particular, $f$ is Lipschitz continuous on $\bar{B}_{e}\left(x_{o}, R / 2\right)$.

We note explicitly that the convexity of $f$ guarantees that $\mathcal{M} \geq f\left(x_{o}\right)$. Moreover, from the first inequality in Theorem 11.3 one obtains the estimate $(11.2)$

$|f(y)| \leq\left[\mathcal{M}-f\left(x_{o}\right)\right]+\left|f\left(x_{o}\right)\right| \leq \mathcal{M}+\left|f\left(x_{o}\right)\right|, \quad$ for every $y \in \bar{B}_{e}\left(x_{o}, R\right)$.

We now return to the Heisenberg group $\mathbb{H}^{n}$.

Theorem 11.4. Let $u: \mathbb{H}^{n} \rightarrow \mathbb{R}$ be a $H$-convex function, then $u \in$ $L_{l o c}^{\infty}\left(\mathbb{H}^{n}\right)$. 
Proof. We present the proof in the case of the first Heisenberg group $\mathbb{H}^{1}$. The case $n \geq 2$ only requires minor modifications, which are left to the reader. Consider the four points

$$
e_{1}^{+}=(1,0,0), \quad e_{1}^{-}=(-1,0,0), \quad e_{2}^{+}=(0,1,0), \quad e_{2}^{+}=(0,1,0) .
$$

The four straight line segments

$$
\begin{aligned}
L_{1}^{+}=(1, s,-2 s), & L_{1}^{-}=(-1, s, 2 s), \\
L_{2}^{+}=(s, 1,2 s), & L_{2}^{-}=(s,-1,-2 s),
\end{aligned}
$$

with $-1 \leq s \leq 1$, are respectively compact subsets of the horizontal planes $H_{e_{1}^{+}}, H_{e_{1}^{-}}, H_{e_{2}^{+}}, H_{e_{2}^{-}}$. By the hypothesis, $u$ restricted to each of these four planes is an ordinary convex function. Appealing to the inequality (11.2) we infer the existence of four constants $M_{i}^{ \pm}>0, i=1,2$, such that

$$
|u(g)| \leq M_{i}^{ \pm}, \quad \text { for every } g \in L_{i}^{ \pm}, i=1,2 .
$$

Setting $M=\max \left\{M_{1}^{+}, M_{1}^{-}, M_{2}^{+}, M_{2}^{-}\right\}$, we conclude

$$
|u(g)| \leq M, \quad \text { for every } g \in L_{1}^{+} \cup L_{1}^{-} \cup L_{2}^{+} \cup L_{2}^{-} .
$$

Having established (11.3) we now consider for every $-1 \leq t \leq 1$ the point $g_{t}=(0,0, t)$ and the horizontal plane $H_{g_{t}}=\left\{g=(x, y, t) \mid(x, y) \in \mathbb{R}^{2}\right\}$. Such plane intersects the segments $L_{1}^{ \pm}, L_{2}^{ \pm}$in the four points

$$
\begin{gathered}
P_{1}^{+}(t)=\left(1,-\frac{t}{2}, t\right), \quad P_{1}^{-}(t)=\left(-1, \frac{t}{2}, t\right), \\
P_{2}^{+}(t)=\left(\frac{t}{2}, 1, t\right), \quad P_{2}^{-}(t)=\left(-\frac{t}{2},-1, t\right) .
\end{gathered}
$$

We now consider the four additional points

$$
\tilde{e}_{1}^{ \pm}=\left( \pm \frac{1}{2}, 0,0\right) \quad \tilde{e}_{2}^{ \pm}=\left(0, \pm \frac{1}{2}, 0\right)
$$

and construct corresponding straight lines segments

$$
\tilde{L}_{1}^{ \pm}=\left( \pm \frac{1}{2}, s, \mp s\right), \quad \tilde{L}_{2}^{ \pm}=\left(s, \pm \frac{1}{2}, \pm s\right), \quad-1 \leq s \leq 1,
$$

as above. Such segments intersect the plane $H_{g_{t}}$ in the four points

$$
\tilde{P}_{1}^{ \pm}(t)=\left( \pm \frac{1}{2}, \mp t, t\right), \quad \tilde{P}_{2}^{ \pm}(t)=\left( \pm t, \pm \frac{1}{2}, t\right) .
$$


Denote by $\tilde{S}(t)$ the simplex in the plane $H_{g_{t}}$ generated by these four points. A moment's thought and elementary geometric considerations will convince the reader that

$$
U=\bigcup_{-1 \leq t \leq 1} \tilde{S}(t)
$$

constitutes a full neighborhood of the group identity $e$, hence there exists $\delta>0$ such that

$$
\bar{B}(e, \delta) \subset U
$$

Similarly to (11.3), there exists a constant $\tilde{M}>0$ such that

$$
|u(g)| \leq \tilde{M}, \quad \text { for every } g \in \tilde{L}_{1}^{+} \cup \tilde{L}_{1}^{-} \cup \tilde{L}_{2}^{+} \cup \tilde{L}_{2}^{-} .
$$

Fix $t \in[-1,1]$. Applying the estimate (11.2) we infer that

$$
\sup _{g \in \tilde{S}(t)}|u(g)| \leq C(t)
$$

where $C(t)>0$ is a constant which depends only on

$$
\max \left\{u\left(P_{1}^{+}(t)\right), u\left(P_{1}^{-}(t)\right), u\left(P_{2}^{+}(t)\right), u\left(P_{2}^{-}(t)\right), \mid u\left(\tilde{P}_{o}(t) \mid\right\},\right.
$$

where $\tilde{P}_{o}(t)$ is an arbitrarily fixed point in $\tilde{S}(t)$, for instance $\tilde{P}_{1}^{+}(t)$. However, the two estimates (11.3) and (11.5) guarantee that

$$
\sup _{-1 \leq t \leq 1} C(t) \leq C=C(M, \tilde{M})<\infty .
$$

From these considerations, we conclude that

$$
\sup _{B(e, \delta)}|u| \leq C(M, \tilde{M})
$$

This proves that $u \in L^{\infty}(B(e, \delta))$. Since by left-translation we can move any point to the group identity, the conclusion of the theorem follows.

Remark 11.5. It should be clear to the reader that by suitably varying the choice of the points $e_{i}^{ \pm}$in the proof of Theorem 11.4 we can make the radius $\delta$ in the estimate (11.7) arbitrarily large.

Theorem 11.6. Let $u: \mathbb{H}^{n} \rightarrow \mathbb{R}$ be a $H$-convex function, then $u \in C\left(\mathbb{H}^{n}\right)$. 
Proof. Again, we present the proof only for $\mathbb{H}^{1}$, and leave the minor adjustments for $n \geq 2$ to the reader. By left-translation it will suffice to prove that $u$ is continuous at the group identity $e=(0,0,0)$. From Theorem 11.4, and Remark 11.5 after it, we know that $u \in L^{\infty}(B(e, 1))$. Set $M=$ $\|u\|_{L^{\infty}((B(e, 1))}$. We will prove that there exist $\eta_{o}=\eta_{o}>0$, and $C=C(M)>$ 0 such that for every $g_{o}=\left(x_{o}, y_{o}, t_{o}\right) \in B\left(e, \eta_{o}\right)$ we have

$$
\left|u\left(g_{o}\right)-u(e)\right| \leq C d\left(g_{o}, e\right) .
$$

We first consider the case when $g_{o}$ belongs to the center of the group, i.e., $g_{o}=\left(0,0, t_{o}\right)$, and without loss of generality we assume $t_{o}>0$. Furthermore, we impose that $t_{o} \leq \eta_{o}^{2}$, where $\eta_{o}>0$ will be suitably chosen in the sequel. Consider the point $\left(-\frac{\sqrt{t_{o}}}{2}, 0,0\right) \in H_{e}$, and the straight-line segment $L_{1}\left(t_{o}\right)$ lying on $H_{e}$ which joins $e$ to such point. The horizontal plane $H_{\left(-\frac{\sqrt{t_{o}}}{2}, 0,0\right)}$ has equation $t=\sqrt{t_{o}} y$. On such plane we consider the straight-line segment $L_{2}\left(t_{o}\right)$ which joins the points $\left(-\frac{\sqrt{t_{o}}}{2}, 0,0\right)$ and $\left(-\frac{\sqrt{t_{o}}}{2}, \sqrt{t_{o}}, t_{o}\right)$. Finally, denote by $L_{3}\left(t_{o}\right)$ the straight-line segment lying on the horizontal plane $H_{\left(0,0, t_{o}\right)}$ joining $\left(-\frac{\sqrt{t_{o}}}{2}, \sqrt{t_{o}}, t_{o}\right)$ to $\left(0,0, t_{o}\right)$. We have

$$
\begin{aligned}
\left|u\left(g_{o}\right)-u(e)\right| & \leq\left|u\left(0,0, t_{o}\right)-u\left(-\frac{\sqrt{t_{o}}}{2}, \sqrt{t_{o}}, t_{o}\right)\right| \\
& +\left|u\left(-\frac{\sqrt{t_{o}}}{2}, \sqrt{t_{o}}, t_{o}\right)-u\left(-\frac{\sqrt{t_{o}}}{2}, 0,0\right)\right| \\
& +\left|u\left(-\frac{\sqrt{t_{o}}}{2}, 0,0\right)-u(0,0,0)\right| \\
& =\left|U_{\left(0,0, t_{o}\right)}(0,0)-U_{\left(0,0, t_{o}\right)}\left(-\frac{\sqrt{t_{o}}}{2}, \sqrt{t_{o}}\right)\right| \\
& +\left|U_{\left(-\frac{\sqrt{t_{o}}}{2}, 0,0\right)}\left(-\frac{\sqrt{t_{o}}}{2}, \sqrt{t_{o}}\right)-U_{\left(-\frac{\sqrt{t_{o}}}{2}, 0,0\right)}\left(-\frac{\sqrt{t_{o}}}{2}, 0\right)\right| \\
& +\left|U_{(0,0,0)}\left(-\frac{\sqrt{t_{o}}}{2}, 0\right)-U_{(0,0,0)}(0,0)\right|
\end{aligned}
$$

where we have denoted by $U_{g_{o}}(x, y)$ the restriction of the function $u(x, y, t)$ to the horizontal plane $H_{g_{o}}$. By the assumption, each of the functions $U_{\left(0,0, t_{o}\right)}(x, y), U_{\left(-\frac{\sqrt{t_{o}}}{2}, 0,0\right)}(x, y), U_{(0,0,0)}(x, y)$ is convex, and therefore locally Lipschitz. As $t_{o}$ varies in the interval $\left[0, \eta_{o}^{2}\right]$, the segments $L_{1}\left(t_{o}\right), L_{2}\left(t_{o}\right)$ and $L_{3}\left(t_{o}\right)$ respectively vary in compact subsets $K_{1}\left(\eta_{o}\right), K_{2}\left(\eta_{o}\right), K_{3}\left(\eta_{o}\right)$ of the horizontal planes $H_{(0,0,0)}, H_{\left(-\frac{\sqrt{t_{o}}}{2}, \sqrt{t_{o}}, t_{o}\right)}, H_{\left(0,0, t_{o}\right)}$. We now choose $\eta$ in such a way that $K_{i}\left(\eta_{o}\right) \subset B\left(e, \frac{1}{2}\right), i=1,2,3$. In view of Theorem 11.3 the local 
Lipschitz constants of the functions $U$ 's on such compact sets are respectively bounded by the maximum of their absolute values on finite simplices which enclose them. But by our choice of $\eta_{o}$ we can choose these simplices so that they are all contained in $B(e, 1)$, and therefore the local Lipschitz constants are uniformly bounded by $M$. We conclude from (11.8)

$$
\left|u\left(g_{o}\right)-u(e)\right| \leq M\left\{\frac{\sqrt{5}}{2} \sqrt{t_{o}}+\sqrt{t_{o}}+\frac{1}{2} \sqrt{t_{o}}\right\}=C(M) d\left(g_{o}, e\right) .
$$

We next consider the case in which $g_{o}=\left(x_{o}, y_{o}, t_{o}\right)$ does not belong to the center, i.e., $x_{o}^{2}+y_{o}^{2} \neq 0$. We now assume that $x_{o}^{2}+y_{o}^{2} \leq \eta_{o}^{2}, 0 \leq t_{o} \leq \eta_{o}^{2}$, where as before we are going to choose $\eta_{o}$ sufficiently small. In the present case we first move along the same polygonal path $L_{1}\left(t_{o}\right) \cup L_{2}\left(t_{o}\right) \cup L_{3}\left(t_{o}\right)$ as before, joining the identity to the point $\left(0,0, t_{o}\right)$. Once we reach $\left(0,0, t_{o}\right)$, we move from there to the point $\left(x_{o}, y_{o}, t_{o}\right)$ along a straight-line segment $L_{4}\left(x_{o}, y_{o}, t_{o}\right)$ lying on the horizontal plane $H_{\left(0,0, t_{o}\right)}$. Similarly to before we obtain

$$
\begin{aligned}
\left|u\left(g_{o}\right)-u(e)\right| & \leq\left|u\left(x_{o}, y_{o}, t_{o}\right)-u\left(0,0, t_{o}\right)\right| \\
& +\left|u\left(0,0, t_{o}\right)-u\left(-\frac{\sqrt{t_{o}}}{2}, \sqrt{t_{o}}, t_{o}\right)\right| \\
& +\left|u\left(-\frac{\sqrt{t_{o}}}{2}, \sqrt{t_{o}}, t_{o}\right)-u\left(-\frac{\sqrt{t_{o}}}{2}, 0,0\right)\right| \\
& +\left|u\left(-\frac{\sqrt{t_{o}}}{2}, 0,0\right)-u(0,0,0)\right| \\
& =\left|U_{\left(0,0, t_{o}\right)}\left(x_{o}, y_{o}\right)-\right| U_{\left(0,0, t_{o}\right)}(0,0) \mid \\
& +\left|U_{\left(0,0, t_{o}\right)}(0,0)-U_{\left(0,0, t_{o}\right)}\left(-\frac{\sqrt{t_{o}}}{2}, \sqrt{t_{o}}\right)\right| \\
& +\left|U_{\left(-\frac{\sqrt{t_{o}}}{2}, 0,0\right)}\left(-\frac{\sqrt{t_{o}}}{2}, \sqrt{t_{o}}\right)-U_{\left(-\frac{\sqrt{t_{o}}}{2}, 0,0\right)}\left(-\frac{\sqrt{t_{o}}}{2}, 0\right)\right| \\
& +\left|U_{(0,0,0)}\left(-\frac{\sqrt{t_{o}}}{2}, 0\right)-U_{(0,0,0)}(0,0)\right| .
\end{aligned}
$$

At this point, the same argument given in the case in which $g_{o}$ is on the center allows to infer

$$
\left|U_{\left(0,0, t_{o}\right)}\left(x_{o}, y_{o}\right)-\right| U_{\left(0,0, t_{o}\right)}(0,0) \mid \leq M \sqrt{x_{o}^{2}+y_{o}^{2}} \leq M d\left(g_{o}, e\right) .
$$

The remaining three terms in the right-hand side of (11.9) are estimated as before. We conclude

$$
\left|u\left(g_{o}\right)-u(e)\right| \leq C(M) d\left(g_{o}, e\right) .
$$


This proves the continuity of $u$ at $e$.

The following lemma provides a useful stability property of $H$-convex functions with respect to the group convolution.

Lemma 11.7. Let $K \in L^{1}(\boldsymbol{G})$ with compact support, and assume $K \geq 0$. If $u: \mathbb{H}^{n} \rightarrow \mathbb{R}$ is $H$-convex, then also $K \star u$ is $H$-convex.

Proof. Recall that

$$
K \star u(g)=\int_{\mathbb{H}^{n}} K(h) u\left(h^{-1} g\right) d h .
$$

Let $v=K \star u$, and fix $g_{o}=\left(x_{o}, y_{o}, t_{o}\right) \in \mathbb{H}^{n}$. Denoting by $V_{g_{o}}$ the restriction of $v$ to $H_{g_{o}}$, we want to show that

$$
V_{g_{o}}\left(x+\lambda\left(x^{\prime}-x\right), y+\lambda\left(y^{\prime}-y\right)\right) \leq(1-\lambda) V_{g_{o}}(x, y)+\lambda V_{g_{o}}\left(x^{\prime}, y^{\prime}\right) .
$$

If $h=(\xi, \eta, \tau)$, and if $U_{g}$ indicates the restriction of $u$ to the horizontal plane $H_{g}$, then a computation shows that

$$
\begin{aligned}
V_{g_{o}}(x, y) & =\int_{\mathbb{H}^{n}} K(h) U_{h^{-1} g_{o}}(-\xi+x,-\eta+y) d h \\
& =\int_{\mathbb{H}^{n}} K(h) \tau_{(-\xi,-\eta)} U_{h^{-1} g_{o}}(x, y) d h,
\end{aligned}
$$

where for a function $f(x, y)$ we have let $\tau_{(-\xi,-\eta)} f(x, y)=f(-\xi+x,-\eta+y)$. By the hypothesis on $u$, for any fixed $h \in \mathbb{H}^{n}$ the function $U_{h^{-1}} g_{o}$ is a convex function of the variables $(x, y) \in \mathbb{R}^{2 n}$, therefore such is also the function $\tau_{(-\xi,-\eta)} U_{h^{-1} g_{o}}$. This implies

$$
\begin{aligned}
\tau_{(-\xi,-\eta)} U_{h^{-1} g_{o}}\left(x+\lambda\left(x^{\prime}-x\right), y+\lambda\left(y^{\prime}-y\right)\right) & \leq(1-\lambda) \tau_{(-\xi,-\eta)} U_{h^{-1} g_{o}}(x, y) \\
& +\lambda \tau_{(-\xi,-\eta)} U_{h^{-1} g_{o}}\left(x^{\prime}, y^{\prime}\right),
\end{aligned}
$$

for any $0 \leq \lambda \leq 1$. Multiplying this inequality by $K(h)$, integrating over $\mathbb{H}^{n}$, and keeping (11.11) in mind, we obtain (11.10). This completes the proof.

It is now clear that in view of Proposition 11.2 and of Theorem 11.6, all the results in this paper continue to be valid, without any additional assumption, for functions which are $H$-convex. Without repeating all the relevant statements we confine ourselves to state the fundamental $L^{\infty}-L^{1}$ estimate for $H$-convex functions. 
Theorem 11.8. Let $u: \mathbb{H}^{n} \rightarrow \mathbb{R}$ be $H$-convex, then there exists $C=$ $C(n)>0$ such that for every ball $B(g, r)$ one has

$$
\sup _{B(g, r)}|u| \leq C \frac{1}{|B(g, 5 r)|} \int_{B(g, 5 r)}|u| d g^{\prime}
$$

and

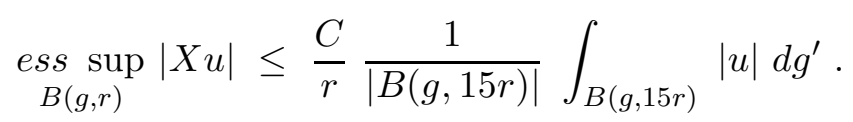

\section{References.}

[A] A. D. Alexandrov, Certain estimates for the Dirichlet problem, Soviet Math. Dokl., 1 (1960), 1151-1154.

[AM] L. Ambrosio \& V. Magnani, Some fine properties of BV functions on sub-Riemannian groups, preprint, 2002.

[Ba1] I. Ja. Bakelman, Theory of quasilinear elliptic equations, Sibirsk. Mat. Ž. (in Russian), 2 (1961), 179-186.

[Ba2] Geometric methods for solving elliptic equations, "Nauka", Moscow, 1965.

[Ba3] Convex Analysis and Nonlinear Geometric Elliptic Equations, Springer-Verlag, 1994.

[Be] A. Bellä̈che \& J.-J. Risler, ed., Sub-Riemannian Geometry, Birkhäuser, 1996.

[Bi] T. Bieske, On $\infty$-harmonic functions on the Heisenberg Group, preprint, 2000.

[CC] L. A. Caffarelli, X. Cabré, Fully Nonlinear Elliptic Equations, Amer. Math. Soc., Colloquium Publ., vol.43, 1991.

[CDG] L. Capogna, D. Danielli \& N. Garofalo, Capacitary estimates and the local behavior of solutions of nonlinear subelliptic equations, Amer. J. Math., 118 (1996), no.6, 1153-1196.

[CGP] L. Capogna, N. Garofalo \& S. Pauls, Asymptotic behavior of the Gauss map near weakly singular characteristic points, preprint, 2002 . 
[Ca] C. Carathéodory, Untersuchungen über die Grundlangen der Thermodynamik, Math. Ann., 67 (1909), 355-386.

[Ch] W. L. Chow, Über Systeme von linearen partiellen Differentialgleichungen erster Ordnung, Math. Annalen, 117 (1939), 98-105.

[CDKR] M. Cowling, A. H. Dooley, A. Korányi \& F. Ricci, H-type groups and Iwasawa decompositions, Adv. Math., 87 (1991), 1-41.

[CIL] M. Crandall, H. Ishii \& P. L. Lions, User's guide to viscosity solutions of second order partial differential equations, Bull. Amer. Math. Soc., 27 (1992), no.1, 1-67.

[Cy] J. Cygan, Subadditivity of homogeneous norms on certain nilpotent Lie groups, Proc. Amer. Math. Soc., 83 (1981), 69-70.

[DGN1] D. Danielli, N. Garofalo \& D. M. Nhieu, Non-doubling Ahlfors measures, perimeter measures, and the characterization of the trace spaces of Sobolev functions in Carnot-Carathéodory spaces, submitted paper, 2002.

[DGN2] Minimal surfaces, area integral, sets of constant mean curvature and isoperimetry in Carnot groups, preprint, 2000.

[DGN3] — The Monge-Ampère equation in Carnot groups, work in preparation.

[DGN4] On the best possible character of the $L^{Q}$ norm in some a priori estimates for non-divergence form equations in Carnot groups, Proceeding of the AMS, to appear.

[DGS] D. Danielli, N. Garofalo \& S. Salsa, Variational inequalities with lack of ellipticity. Part I: optimal interior regularity and nondegeneracy of the free boundary, Indiana Univ. Math. J., to appear.

[Du] R. M. Dudley, On second derivatives of convex functions, Math. Scand., 41 (1977), 159-174.

[EG] L. C. Evans \& R. F. Gariepy, Measure Theory and Fine Properties of Functions, CRC press, 1992.

[F1] G. Folland, A fundamental solution for a subelliptic operator, Bull. Amer. Math. Soc., 79 (1973), 373-376. 
[F2] G. B. Folland, Subelliptic estimates and function spaces on nilpotent Lie groups, Ark. Math., 13 (1975), 161-207.

[FS] G. B. Folland \& E. M. Stein, Estimates for the $\bar{\partial}_{b}$ Complex and Analysis on the Heisenberg Group, Comm. Pure Appl. Math., 27 (1974), 429-522.

[FSS] B. Franchi, R. Serapioni \& F. Serra Cassano, Approximation and imbedding theorems for weighted Sobolev spaces associated with Lipschitz continuous vector fields, Boll. Un. Mat. Ital. B (7) 11 (1997), no. 1, 83-117.

[G] N. Garofalo, Analysis and Geometry of Carnot-Carathéodory Spaces, With Applications to Pde's, Birkhäuser, book in preparation.

[GN1] N. Garofalo \& D. M. Nhieu, Isoperimetric and Sobolev inequalities for Carnot-Carathéodory spaces and the existence of minimal surfaces, Comm. Pure Appl. Math., 49 (1996), 1081-1144.

[GN2] , Lipschitz continuity, global smooth approximations and extension theorems for Sobolev functions in CarnotCarathéodory spaces, J. Anal. Math., 74 (1998), 67-97.

[GP] N. Garofalo \& S. D. Pauls, The Bernstein problem in the Heisenberg group, preprint, 2002.

[GTo] N. Garofalo \& F. Tournier, Monotonicity and comparison theorems for Monge-Ampère measures in the Heisenberg group, preprint.

[GV1] N. Garofalo \& D. Vassilev, Symmetry properties of positive entire solutions of Yamabe-type equations on groups of Heisenberg type, Duke Math. J., 106 (2001), 411-448.

[GV2] Ltrong unique continuation for some subelliptic operators, preprint, 1999.

[GT] D. Gilbarg \& N. S. Trudinger, Elliptic Partial Differential Equations of Second Order, Second Ed., Springer, 1998.

[Gu] C. E. Gutierrez, The Monge-Ampère Equation, Birkhäuser, 2001.

[HH] J. Heinonen \& I. Holopainen, Quasiregular maps on Carnot groups, J. Geom. Anal., 7 (1997), no.1, 109-148. 
[H1] H. Hörmander, Hypoelliptic second-order differential equations, Acta Math., 119 (1967), 147-171.

[H2] Notions of convexity, Birkhäuser, 1994.

[K] A. Kaplan, Fundamental solutions for a class of hypoelliptic PDE generated by composition of quadratic forms, Trans. Amer. Math. Soc., 258 (1980), 147-153.

[LMS] G. Lu, J. Manfredi \& B. Stroffolini, Convex functions on the Heisenberg group, preprint, June 2002.

[MSC] R. Monti \& F. Serra Cassano, Surface measures in CarnotCarathéodory spaces, Calc. Var. Partial Differential Equations, 13 (2001), no. 3, 339-376.

[P] P. Pansu, Métriques de Carnot-Carathéodory et quasi-isométries des espaces symétriques de rang un, Ann. of Math. (2)129 (1989),1, $1-60$.

[Po] A. V. Pogorelov, Extrinsic Geometry of Convex Surfaces, Amer. Math. Soc., 1973.

[Pu] C. Pucci, Limitazioni per soluzioni di equazioni ellittiche, Ann. Mat. Pura Appl., (4) 74 (1966), 15-30.

[R] J. G. Rešetnjak, Generalized derivatives and differentiability almost everywhere, Math. USSR - Sbornik, 4 (1968), no.3, 293-302.

[Ro] R. T. Rockafellar, Convex Analysis, Princeton Univ. Press, 1970.

[Sch] R. Schneider, Convex Bodies: The Brunn-Minkowski Theory, Cambridge Univ. Press, 1993.

[S] E. M. Stein, Harmonic Analysis: Real Variable Methods, Orthogonality and Oscillatory Integrals, Princeton Univ. Press, 1993.

[V] V. S. Varadarajan, Lie Groups, Lie Algebras, and Their Representations, Springer-Verlag, New York, Berlin, Heidelberg, Tokyo, 1974.

Department of Mathematics

Purdue University

West LAFAYETte, IN 47907

E-mail: danielli@math.purdue.edu 
Department of Mathematics

Purdue University

WEST LAFAYETTE, IN 47907

E-mail: garofalo@math.purdue.edu

Dipartimento di Metodi e Modelli Matematici Per le Scienze Applicate UnIVERSitÀ di PADOVA

35131 PAdOVA, ITALY

E-mail: garofalo@dmsa.unipd.it

Department of Mathematics

GEORGETOWN UnIVERSITY

WASHINGTON DC 20057-1233

E-mail: nhieu@math.georgetown.edu 